\title{
Attrition-Enhanced Deracemization and Absolute Asymmetric Synthesis of Flavanones from Prochiral Precursors
}

Waku Shimizu, ${ }^{\dagger}$ Naohiro Uemura, ${ }^{\dagger}$ Yasushi Yoshida, ${ }^{\dagger, \ddagger}$ Takashi Mino, ${ }^{\dagger, \ddagger}$ Yoshio Kasashima, ${ }^{\S}$ and Masami Sakamoto,,,$+ \ddagger$

† Department of Applied Chemistry and Biotechnology, Graduate School of Engineering Chiba University, Yayoi-Cho, Inage-Ku, Chiba 263-8522, Japan

‡ Molecular Chirality Research Center, Chiba University, Yayoi-Cho, Inage-Ku, Chiba 263-8522, Japan

$\S$ Education Center, Faculty of Creative Engineering, Chiba Institute of Technology

Shibazono, Narashino, Chiba 275-0023, Japan 


\section{Table of Contents}

General

Synthesis of aldol reaction product 3

Synthesis of flavanones 4 S6

Single crystal X-ray structure analysis of 4 S7

Attrition-enhanced deracemization racemic flavanones 4 $\mathrm{S} 20$

One-pot absolute asymmetric synthesis of $\mathbf{4 m}$ from $\mathbf{3 m}$ $\mathrm{S} 20$

One-pot absolute asymmetric synthesis of $\mathbf{4 m}$ from $\mathbf{1}$ and $\mathbf{2 m}$ $\mathrm{S} 20$

References S21

HPLC analysis of 4 S22

NMR spectra of 3 S32

NMR spectra of 4 S46 


\section{General}

NMR spectra were recorded in $\mathrm{CDCl}_{3}$ solutions on Bruker DPX 300 and DPX 400 spectrometers for ${ }^{1} \mathrm{H}$ - and ${ }^{13} \mathrm{C}$ NMR. Chemical shifts are reported in parts per million $(\mathrm{ppm})$ relative to TMS as an internal standard. IR spectra were recorded on a JASCO FT/IR-230 spectrometer. HPLC analyses were performed on a JASCO HPLC system (JASCO PU-1580 pump, DG-1580-53, LG-2080-02, MD-2015, UV-2075, and CD-2095 detector). High-resolution mass spectrometry (HRMS) was performed on an Orbitrap ThermoFisher Exactive ion trap mass spectrometer. Single crystal X-ray structure analysis was conducted using a SMART APEX II (Bruker AXS) and APEX II ULTRA (Bruker AXS). Commercially available 4,6-dimethoxy-2-hydroxyacetophenone, aromatic aldehydes, and DBU were used without further purification. Chiral column CHIRALPAK IB (Daicel Ind.) were used for HPLC analysis.

\section{Synthesis of aldol reaction product 3}

A methanol or ethanol solution of 4,6-dimethoxy-2-hydroxyacetophenone 1, aromatic aldehyde 2 (1.0 equiv), and sodium hydroxide (1.5 - 5.0 equiv) was stirred at room temperature for 24 hours. Ethyl acetate was added and the mixture was washed with hydrochloric acid and saturated brine, the organic layer was dried over magnesium sulfate, and the solvent was removed under reduced pressure to give the crude product 3 . The crude mixture was separated by column chromatography (eluent: ethyl acetate/hexane) to afford corresponding adduct 3 . Crystalline products were recrystallized from $\mathrm{CHCl}_{3}$ and hexane. As an example, when 1 (1.88 g, $\left.9.6 \mathrm{mmol}\right), 2 \mathrm{~m}(1.4 \mathrm{~mL}, 9.6 \mathrm{mmol})$, and sodium hydroxide $(1.2 \mathrm{~g}, 30.0 \mathrm{mmol})$ were used, $3 \mathrm{~m}$ was obtained in $73 \%$ yield.

(E)-1-(2-Hydroxy-4,6-dimethoxyphenyl)-3-phenylprop-2-en-1-one 3a. ${ }^{[\mathrm{S1}]} 80 \%$ yield; yellow crystal; ${ }^{1} \mathrm{H}$ NMR $\left(\mathrm{CDCl}_{3}\right)$ $\delta 3.83(\mathrm{~s}, 3 \mathrm{H}), 3.92(\mathrm{~s}, 3 \mathrm{H}), 5.96(\mathrm{~d}, J=2.0 \mathrm{~Hz}, 1 \mathrm{H}), 6.11(\mathrm{~d}, J=2.4 \mathrm{~Hz}, 1 \mathrm{H}), 7.38-7.43(\mathrm{~m}, 3 \mathrm{H}), 7.60(\mathrm{dd}, J=7.4$ and $2.2 \mathrm{~Hz}, 2 \mathrm{H}), 7.78(\mathrm{~d}, J=15.6 \mathrm{~Hz}, 1 \mathrm{H}), 7.90(\mathrm{~d}, J=15.6 \mathrm{~Hz}, 1 \mathrm{H}) ;{ }^{13} \mathrm{C} \mathrm{NMR}\left(\mathrm{CDCl}_{3}\right) \delta 55.6,55.8,91.2,93.8$, 106.3, 127.5, 128.4, 128.8, 130.0, 135.5, 142.3, 162.5, 166.2, 168.4, 192.6.

(E)-1-(2-Hydroxy-4,6-dimethoxyphenyl)-3-(p-tolyl)prop-2-en-1-one 3c. $65 \%$ yield; yellow crystal; m.p. $133-134{ }^{\circ} \mathrm{C}$; IR $\left(\mathrm{cm}^{-1}, \mathrm{KBr}\right) 1630,3438 ;{ }^{1} \mathrm{H}$ NMR $\left(\mathrm{CDCl}_{3}\right) \delta 3.83(\mathrm{~s}, 3 \mathrm{H}), 3.91(\mathrm{~s}, 3 \mathrm{H}), 5.96(\mathrm{~d}, J=2.8 \mathrm{~Hz}, 1 \mathrm{H}), 6.10(\mathrm{~d}, J=2.0$ $\mathrm{Hz}, 1 \mathrm{H}), 7.21(\mathrm{~d}, J=8.0 \mathrm{~Hz}, 2 \mathrm{H}), 7.50(\mathrm{~d}, J=8.0 \mathrm{~Hz}, 2 \mathrm{H}), 7.77(\mathrm{~d}, J=15.6 \mathrm{~Hz}, 1 \mathrm{H}), 7.87(\mathrm{~d}, J=15.6 \mathrm{~Hz}, 1 \mathrm{H}) ;{ }^{13} \mathrm{C}$ $\operatorname{NMR}\left(\mathrm{CDCl}_{3}\right) \delta 21.5,55.6,55.8,91.2,93.8,106.3,126.5,128.4,129.6,132.8,140.5,142.5,162.4,166.1,168.3$, 192.7; HRMS (ESI-MS) $\mathrm{m} / \mathrm{z}$ calcd for $\mathrm{C}_{18} \mathrm{H}_{18} \mathrm{O}_{4}+\mathrm{H} 299.1278$, found 299.1270 .

(E)-3-(4-Fluoromethylphenyl)-1-(2-hydroxy-4,6-dimethoxyphenyl)prop-2-en-1-one $\mathbf{3 h}$. $70 \%$ yield; yellow crystal; m.p. $144-145{ }^{\circ} \mathrm{C}$; IR ( $\left.\mathrm{cm}^{-1}, \mathrm{KBr}\right) 1644,3448 ;{ }^{1} \mathrm{H} \mathrm{NMR}\left(\mathrm{CDCl}_{3}\right) \delta 3.84(\mathrm{~s}, 3 \mathrm{H}), 3.92(\mathrm{~s}, 3 \mathrm{H}), 5.97(\mathrm{~d}, \mathrm{~J}=2.4 \mathrm{~Hz}, 1 \mathrm{H})$, $6.11(\mathrm{~d}, J=2.4 \mathrm{~Hz}, 1 \mathrm{H}), 7.10(\mathrm{t}, J=8.8 \mathrm{~Hz}, 2 \mathrm{H}), 7.59(\mathrm{q}, J=4.8 \mathrm{~Hz}, 2 \mathrm{H}), 7.74(\mathrm{~d}, J=15.6 \mathrm{~Hz}, 1 \mathrm{H}), 7.83(\mathrm{~d}, J=$ $15.6 \mathrm{~Hz}, 1 \mathrm{H}) ;{ }^{13} \mathrm{C} \mathrm{NMR}\left(\mathrm{CDCl}_{3}\right) \delta 55.6,55.9,92.3,93.8,106.2,115.9,116.1,127.2(\mathrm{~d}, J=1.4 \mathrm{~Hz}), 130.1(\mathrm{~d}, J=8.0$ $\mathrm{Hz}), 131.8\left(\mathrm{~d}, \mathrm{~J}=2.8 \mathrm{~Hz}\right.$ ), 141.0, 162.4, 166.2, 168.4, 192.4; HRMS (ESI-MS) $\mathrm{m} / \mathrm{z}$ calcd for $\mathrm{C}_{17} \mathrm{H}_{15} \mathrm{FO}_{4}+\mathrm{H}$ 303.1027, found 303.1022.

(E)-3-(4-Chlorophenyl)-1-(2-hydroxy-4,6-dimethoxyphenyl)prop-2-en-1-one 3j. 73\% yield; yellow crystal; m.p. 176-177 ${ }^{\circ} \mathrm{C}$; IR ( $\left.\mathrm{cm}^{-1}, \mathrm{KBr}\right) 1636,3444$; ${ }^{1} \mathrm{H}$ NMR $\left(\mathrm{CDCl}_{3}\right) \delta 3.84(\mathrm{~s}, 3 \mathrm{H}), 3.92(\mathrm{~s}, 3 \mathrm{H}), 5.97(\mathrm{~d}, \mathrm{~J}=2.4 \mathrm{~Hz}, 1 \mathrm{H}), 6.11$ (d, $J=2.4 \mathrm{~Hz}, 1 \mathrm{H}), 7.38(\mathrm{~d}, J=8.8 \mathrm{~Hz}, 2 \mathrm{H}), 7.53(\mathrm{~d}, J=8.4 \mathrm{~Hz}, 2 \mathrm{H}), 7.72(\mathrm{~d}, J=15.6 \mathrm{~Hz}, 1 \mathrm{H}), 7.86(\mathrm{~d}, J=15.6 \mathrm{~Hz}$, $1 \mathrm{H}) ;{ }^{13} \mathrm{C} \mathrm{NMR}\left(\mathrm{CDCl}_{3}\right) \delta 55.6,55.9,91.3,93.8,106.3,128.0,129.1,129.4,134.1,135.8,140.8,162.4,166.3,168$, 4, 192.3; HRMS (ESI-MS) $\mathrm{m} / \mathrm{z}$ calcd for $\mathrm{C}_{17} \mathrm{H}_{15} \mathrm{ClO}_{4}+\mathrm{H} 319.0732$, found 319.0724 . 
(E)-3-(4-Bromophenyl)-1-(2-hydroxy-4,6-dimethoxyphenyl)prop-2-en-1-one $3 \boldsymbol{k}$. 72\% yield; yellow crystal; m.p. 169-170 ${ }^{\circ} \mathrm{C}$; IR $\left(\mathrm{cm}^{-1}, \mathrm{KBr}\right) 1635,3444 ;{ }^{1} \mathrm{H}$ NMR $\left(\mathrm{CDCl}_{3}\right) \delta 3.84(\mathrm{~s}, 3 \mathrm{H}), 3.91(\mathrm{~s}, 3 \mathrm{H}), 5.96(\mathrm{~d}, \mathrm{~J}=2.4 \mathrm{~Hz}, 1 \mathrm{H}), 6.11$ $(\mathrm{d}, J=2.4 \mathrm{~Hz}, 1 \mathrm{H}), 7.45(\mathrm{~d}, J=8.4 \mathrm{~Hz}, 2 \mathrm{H}), 7.53(\mathrm{~d}, J=8.4 \mathrm{~Hz}, 2 \mathrm{H}), 7.69(\mathrm{~d}, J=15.6 \mathrm{~Hz}, 1 \mathrm{H}), 7.87(\mathrm{~d}, J=15.6 \mathrm{~Hz}$, $1 \mathrm{H}) ;{ }^{13} \mathrm{C}$ NMR $\left(\mathrm{CDCl}_{3}\right) \delta 55.6,55.9,91.3,93.8,106.3,124.2,128.1,129.7,132.1,134.5,140.8,162.4,166.3,168.4$, 192.3; HRMS (ESI-MS) $\mathrm{m} / \mathrm{z}$ calcd for $\mathrm{C}_{17} \mathrm{H}_{15} \mathrm{BrO}_{4}+\mathrm{H} 363.0226$, found 363.0220 .

(E)-1-(2-Hydroxy-4,6-dimethoxyphenyl)-3-(naphthalen-1-yl)prop-2-en-1-one 3I. 72\% yield; yellow crystal; m.p. 143$144{ }^{\circ} \mathrm{C}$; IR ( $\left(\mathrm{cm}^{-1}, \mathrm{KBr}\right) 1633,3412 ;{ }^{1} \mathrm{H}$ NMR $\left(\mathrm{CDCl}_{3}\right) \delta 3.84(\mathrm{~s}, 3 \mathrm{H}), 3.90(\mathrm{~s}, 3 \mathrm{H}), 5.97(\mathrm{~d}, J=2.4 \mathrm{~Hz}, 1 \mathrm{H}), 6.13(\mathrm{~d}, J$ $=2.0 \mathrm{~Hz}, 1 \mathrm{H}), 7.49-7.60(\mathrm{~m}, 3 \mathrm{H}), 7.83(\mathrm{~d}, J=7.2 \mathrm{~Hz}, 1 \mathrm{H}), 7.89(\mathrm{t}, J=6.6 \mathrm{~Hz}, 2 \mathrm{H}), 7.96(\mathrm{~d}, J=15.6 \mathrm{~Hz}, 1 \mathrm{H}), 8.31$ $(\mathrm{d}, J=8.4 \mathrm{~Hz}, 1 \mathrm{H}), 8.61(\mathrm{~d}, J=15.2 \mathrm{~Hz}, 1 \mathrm{H}) ;{ }^{13} \mathrm{C} \mathrm{NMR}\left(\mathrm{CDCl}_{3}\right) \delta 55.6,55.9,91.3,93.8,106.3,125.1,126.2,126.8$, 128.7, 130.2, 130.3, 162.5, 166.3, 168.4, 192.5; HRMS (ESI-MS) $\mathrm{m} / \mathrm{z}$ calcd for $\mathrm{C}_{21} \mathrm{H}_{18} \mathrm{O}_{4}+\mathrm{H} 335.1278$, found 335.1268 .

(E)-1-(2-Hydroxy-4,6-dimethoxyphenyl)-3-(naphthalen-2-yl)prop-2-en-1-one 3m. 73\% yield; yellow crystal; m.p. 145-146 ${ }^{\circ} \mathrm{C}$; IR ( $\left.\mathrm{cm}^{-1}, \mathrm{KBr}\right) 1648,3465 ;{ }^{1} \mathrm{H}$ NMR $\left(\mathrm{CDCl}_{3}\right) \delta 3.84(\mathrm{~s}, 3 \mathrm{H}), 3.94(\mathrm{~s}, 3 \mathrm{H}), 5.98(\mathrm{~d}, \mathrm{~J}=2.4 \mathrm{~Hz}, 1 \mathrm{H}), 6.12$ $(\mathrm{d}, J=2.4 \mathrm{~Hz}, 1 \mathrm{H}), 7.49-7.53(\mathrm{~m}, 2 \mathrm{H}), 7.74-8.03(\mathrm{~m}, 7 \mathrm{H}) ;{ }^{13} \mathrm{C} \mathrm{NMR}\left(\mathrm{CDCl}_{3}\right) \delta 55.6,55.9,91.3,93.8,106.4,126.6$, 127.1, 127.7, 127.7, 128.6, 130.4, 142.4, 162.5, 166.2, 168.4, 192.5; HRMS (ESI-MS) $\mathrm{m} / \mathrm{z}$ calcd for $\mathrm{C}_{21} \mathrm{H}_{18} \mathrm{O}_{4}+\mathrm{H}$ 335.1278 , found 335.1270 .

\section{Synthesis of flavanones 4}

The corresponding aromatic aldehyde 2 (1.0 equiv) and sodium hydroxide (3.0 equiv) were added to a solution of 1 (1.0 equiv) in methanol, and the mixture was stirred in an open system at room temperature until the solvent was completely evaporated. Ethyl acetate and water were added, the mixture was washed with hydrochloric acid and saturated brine, the organic layer was dried over magnesium sulfate, and the solvent was removed under reduced pressure to give a residue containing adduct 3 . The residual mixture was added to a mixed solvent of pyridine, methanol, and water (1: 1: 1), refluxed at $100{ }^{\circ} \mathrm{C}$ for 24 hours, stirred and then cooled to room temperature. The solvent was removed under reduced pressure, ethyl acetate was added, and the organic layer was washed with hydrochloric acid and saturated brine. The organic layer was dried over magnesium sulfate, the solvent was removed under reduced pressure, and the crude product was purified by column chromatography (ethyl acetate/hexane) to afford the corresponding flavanone 4 . As an example, when 1 (200 mg, $1.0 \mathrm{mmol}), 2 \mathrm{~m} \mathrm{(159} \mathrm{mg,} 1.0 \mathrm{mmol})$ and sodium hydroxide $(121 \mathrm{mg}, 3.0 \mathrm{mmol}$ ) were used, $4 \mathrm{~m}$ was obtained in $75 \%$ yield.

5,7-Dimethoxy-2-phenylchroman-4-one 4a.[S2] 58\% yield; colorless crystal; m.p. $147-148{ }^{\circ} \mathrm{C}$ (conglomerate), m.p. 143-144 ${ }^{\circ} \mathrm{C}$ (racemic); ${ }^{1} \mathrm{H}$ NMR $\left(\mathrm{CDCl}_{3}\right) \delta 2.80(\mathrm{dd}, J=16.6$ and $3.0 \mathrm{~Hz}, 1 \mathrm{H}), 3.03(\mathrm{dd}, J=16.6$ and $13.0 \mathrm{~Hz}, 1 \mathrm{H})$, $3.82(\mathrm{~s}, 3 \mathrm{H}), 3.90(\mathrm{~s}, 3 \mathrm{H}), 5.41(\mathrm{dd}, J=13.0$ and $3.0 \mathrm{~Hz}, 1 \mathrm{H}), 6.10(\mathrm{~d}, J=2.4 \mathrm{~Hz}, 1 \mathrm{H}), 6.16(\mathrm{~d}, J=2.0 \mathrm{~Hz}, 1 \mathrm{H})$, 7.35-7.48 (m, 5H); ${ }^{13} \mathrm{C} \mathrm{NMR}\left(\mathrm{CDCl}_{3}\right) \delta 45.6,55.6,56.1,79.2,93.1,93.5,105.9,126.1,128.6,128.7,138.7,162.2$, $164.9,165.9,189.2$.

5,7-Dimethoxy-2-(m-tolyl)chroman-4-one 4b. 53\% yield; colorless crystal; m.p. $147-148{ }^{\circ} \mathrm{C}$; IR (cm $\left.{ }^{-1}, \mathrm{KBr}\right) 1665 ;{ }^{1} \mathrm{H}$ $\operatorname{NMR}\left(\mathrm{CDCl}_{3}\right) \delta 2.39(\mathrm{~s}, 3 \mathrm{H}), 2.78(\mathrm{dd}, J=16.8$ and $2.9 \mathrm{~Hz}, 1 \mathrm{H}), 3.02(\mathrm{dd}, J=16.6$ and $13.4 \mathrm{~Hz}, 1 \mathrm{H}), 3.82(\mathrm{~s}, 3 \mathrm{H})$, $3.90(\mathrm{~s}, 3 \mathrm{H}), 5.37(\mathrm{dd}, J=13.4$ and $2.9 \mathrm{~Hz}, 1 \mathrm{H}), 6.09(\mathrm{~d}, J=2.4 \mathrm{~Hz}, 1 \mathrm{H}), 6.16(\mathrm{~d}, J=2.4 \mathrm{~Hz}, 1 \mathrm{H}), 7.18(\mathrm{~d}, J=7.6$ $\mathrm{Hz}, 1 \mathrm{H}), 7.24(\mathrm{~d}, J=7.2 \mathrm{~Hz}, 1 \mathrm{H}), 7.28-7.33(\mathrm{~m}, 2 \mathrm{H}) ;{ }^{13} \mathrm{C}$ NMR $\left(\mathrm{CDCl}_{3}\right) \delta 21.4,45.6,55.7,56.1,79.3,93.1,93.5$, 105.9, 123.2, 126.8, 128.6, 129.4, 138.5, 138.6, 162.2, 165.0, 165.9, 189.3; HRMS (ESI-MS) $\mathrm{m} / \mathrm{z}$ calcd for $\mathrm{C}_{18} \mathrm{H}_{18} \mathrm{O}_{4}+\mathrm{H} 299.1278$, found 299.1284. 
5,7-Dimethoxy-2-(p-tolyl)chroman-4-one 4c. 60\% yield; colorless crystal; m.p. $165-166{ }^{\circ} \mathrm{C}$ (conglomerate), m.p. 161-162 ${ }^{\circ} \mathrm{C}$ (racemic); IR ( $\left.\mathrm{cm}^{-1}, \mathrm{KBr}\right) 1678 ;{ }^{1} \mathrm{H} \mathrm{NMR}\left(\mathrm{CDCl}_{3}\right) \delta 2.37(\mathrm{~s}, 3 \mathrm{H}), 2.78(\mathrm{dd}, J=16.4$ and $2.9 \mathrm{~Hz}, 1 \mathrm{H}), 3.03$ (dd, $J=16.6$ and $13.0 \mathrm{~Hz}, 1 \mathrm{H}$ ), $3.82(\mathrm{~s}, 3 \mathrm{H}), 3.90(\mathrm{~s}, 3 \mathrm{H}), 5.37(\mathrm{dd}, J=13.0$ and $2.9 \mathrm{~Hz}, 1 \mathrm{H}), 6.09(\mathrm{~d}, J=2.4 \mathrm{~Hz}$, $1 \mathrm{H}), 6.15(\mathrm{~d}, J=2.4 \mathrm{~Hz}, 1 \mathrm{H}), 7.23(\mathrm{~d}, J=8.0 \mathrm{~Hz}, 2 \mathrm{H}), 7.35(\mathrm{~d}, J=8.4 \mathrm{~Hz}, 2 \mathrm{H}) ;{ }^{13} \mathrm{C} \mathrm{NMR}\left(\mathrm{CDCl}_{3}\right) \delta 21.2,45.5,55.7$, 56.1, 79.1, 93.1, 93.5, 106.0, 126.1, 129.4, 135.7, 138.5, 162.2, 165.0, 165.9, 189.4 HRMS (ESI-MS) $\mathrm{m} / \mathrm{z}$ calcd for $\mathrm{C}_{18} \mathrm{H}_{18} \mathrm{O}_{4}+\mathrm{H} 299.1278$, found 299.1285 .

5,7-Dimethoxy-2-(3-methoxyphenyl)chroman-4-one 4d. [S3] 46\% yield; colorless crystal; m.p. 95-96 ${ }^{\circ} \mathrm{C} ;{ }^{1} \mathrm{H}$ NMR $\left(\mathrm{CDCl}_{3}\right) \delta 2.79(\mathrm{dd}, J=16.4$ and $3.0 \mathrm{~Hz}, 1 \mathrm{H}), 3.01(\mathrm{dd}, J=16.4$ and $13.2 \mathrm{~Hz}, 1 \mathrm{H}), 3.82(\mathrm{~s}, 3 \mathrm{H}), 3.84(\mathrm{~s}, 3 \mathrm{H}), 3.90(\mathrm{~s}$, $3 \mathrm{H}$ ), $5.38(\mathrm{dd}, J=13.2$ and $3.0 \mathrm{~Hz}, 1 \mathrm{H}), 6.10(\mathrm{~d}, J=2.0 \mathrm{~Hz}, 1 \mathrm{H}), 6.17(\mathrm{~d}, J=2.4 \mathrm{~Hz}, 1 \mathrm{H}$ ), 6.90 (dd, $J=7.8$ and 2.2 $\mathrm{Hz}, 1 \mathrm{H}), 7.02(\mathrm{~d}, J=7.6 \mathrm{~Hz}, 2 \mathrm{H}), 7.33(\mathrm{t}, J=8.0 \mathrm{~Hz}, 1 \mathrm{H}) ;{ }^{13} \mathrm{C} \mathrm{NMR}\left(\mathrm{CDCl}_{3}\right) \delta$ 45.6, 55.3, 55.6, 56.1, 79.0, 93.1, 93.5, 105.9, 111.7, 114.0, 118.2, 129.8, 140.3, 159.8, 162.2, 164.9, 165.9, 189.1.

5,7-Dimethoxy-2-(4-methoxyphenyl)chroman-4-one 4e.[S2] 52\% yield; colorless crystal; m.p. 91-92 ${ }^{\circ} \mathrm{C} ;{ }^{1} \mathrm{H}$ NMR $\left(\mathrm{CDCl}_{3}\right) \delta 2.76(\mathrm{dd}, J=16.4$ and $3.2 \mathrm{~Hz}, 1 \mathrm{H}), 3.04(\mathrm{dd}, J=18.0$ and $13.2 \mathrm{~Hz}, 1 \mathrm{H}), 3.81(\mathrm{~s}, 3 \mathrm{H}), 3.82(\mathrm{~s}, 3 \mathrm{H}), 3.89(\mathrm{~s}$, $3 \mathrm{H}), 5.35(\mathrm{dd}, J=13.2$ and $3.2 \mathrm{~Hz}, 1 \mathrm{H}), 6.08(\mathrm{~d}, J=1.6 \mathrm{~Hz}, 1 \mathrm{H}), 6.13(\mathrm{~d}, J=1.6 \mathrm{~Hz}, 1 \mathrm{H}), 6.94(\mathrm{~d}, J=7.6 \mathrm{~Hz}, 2 \mathrm{H})$, $7.38(\mathrm{~d}, J=7.2 \mathrm{~Hz}, 2 \mathrm{H}) ;{ }^{13} \mathrm{C}$ NMR $\left(\mathrm{CDCl}_{3}\right) \delta 45.3,55.3,55.5,56.1,78.9,93.0,93.5,105.9,114.1,127.6,130.7$, $159.8,162.2,165.0,165.9,189.4$.

2-(3,4-Dimethoxyphenyl)-5,7-dimethoxychroman-4-one 4f. 44\% yield; colorless crystal; m.p. 161-162 ${ }^{\circ} \mathrm{C}$; IR ( $\mathrm{cm}^{-1}$, $\mathrm{KBr}) 1675 ;{ }^{1} \mathrm{H}$ NMR $\left(\mathrm{CDCl}_{3}\right) \delta 2.78(\mathrm{dd}, J=16.6$ and $2.7 \mathrm{~Hz}, 1 \mathrm{H}), 3.05(\mathrm{dd}, J=16.4$ and $13.2 \mathrm{~Hz}, 1 \mathrm{H}), 3.82(\mathrm{~s}, 3 \mathrm{H})$, $3.90(\mathrm{~s}, 6 \mathrm{H}), 3.93(\mathrm{~s}, 3 \mathrm{H}), 5.35(\mathrm{dd}, J=13.2$ and $2.7 \mathrm{~Hz}, 1 \mathrm{H}), 6.10(\mathrm{~d}, J=2.0 \mathrm{~Hz}, 1 \mathrm{H}), 6.16(\mathrm{~d}, J=2.4 \mathrm{~Hz}, 1 \mathrm{H}), 6.90$ $(\mathrm{d}, J=8.8 \mathrm{~Hz}, 1 \mathrm{H}), 7.00(\mathrm{~d}, J=6.0 \mathrm{~Hz}, 2 \mathrm{H}) ;{ }^{13} \mathrm{C} \mathrm{NMR}\left(\mathrm{CDCl}_{3}\right) \delta 45.5,55.5,55.9,56.1,79.1,93.1,93.5,105.9$, 109.3, 111.0, 118.7, 123.9 (q, $J=270.7 \mathrm{~Hz}), 125.7$ (q, $J=3.6 \mathrm{~Hz}), 130.7$ (q, $J=32.3 \mathrm{~Hz})$ 131.1, 149.1, 149.3, 162.2, 164.9, 165.9, 189.3; HRMS (ESI-MS) $\mathrm{m} / \mathrm{z}$ calcd for $\mathrm{C}_{19} \mathrm{H}_{20} \mathrm{O}_{6}+\mathrm{H} 345.1333$, found 345.1341.

2-(3,5-Dimethoxyphenyl)-5,7-dimethoxychroman-4-one 4 g. $40 \%$ yield; colorless crystal; m.p. 131-132 ${ }^{\circ} \mathrm{C}$; IR $\left(\mathrm{cm}^{-1}\right.$, $\mathrm{KBr}) 1672 ;{ }^{1} \mathrm{H}$ NMR $\left(\mathrm{CDCl}_{3}\right) \delta 2.79(\mathrm{dd}, J=16.4$ and $2.8 \mathrm{~Hz}, 1 \mathrm{H}), 2.99(\mathrm{dd}, J=16.4$ and $13.2 \mathrm{~Hz}, 1 \mathrm{H}), 3.82(\mathrm{~s}, 6 \mathrm{H})$, $3.83(\mathrm{~s}, 3 \mathrm{H}), 3.90(\mathrm{~s}, 3 \mathrm{H}), 5.34(\mathrm{dd}, J=13.2$ and $2.8 \mathrm{~Hz}, 1 \mathrm{H}), 6.10(\mathrm{~d}, J=2.4 \mathrm{~Hz}, 1 \mathrm{H}), 6.17(\mathrm{~d}, J=2.0 \mathrm{~Hz}, 1 \mathrm{H}), 6.45$ $(\mathrm{t}, J=2.2 \mathrm{~Hz}, 1 \mathrm{H}), 6.60(\mathrm{~d}, J=2.0 \mathrm{~Hz}, 2 \mathrm{H}) ;{ }^{13} \mathrm{C} \mathrm{NMR}\left(\mathrm{CDCl}_{3}\right) \delta 45.7,55.4,55.6,56.1,79.1,93.2,93.5,100.3,104.0$, 105.0, 141.1, 161.0, 162.2, 164.8, 165.9, 189.1; HRMS (ESI-MS) $\mathrm{m} / \mathrm{z}$ calcd for $\mathrm{C}_{19} \mathrm{H}_{20} \mathrm{O}_{6}+\mathrm{H} 345.1333$, found 345.1339 .

2-(4-Fluoromethylphenyl)-5,7-dimethoxychroman-4-one 4h. 58\% yield; colorless crystal; m.p. $160-161{ }^{\circ} \mathrm{C}$ (conglomerate), m.p. 153-154 ${ }^{\circ} \mathrm{C}$ (racemic); IR $\left(\mathrm{cm}^{-1}, \mathrm{KBr}\right) 1679 ;{ }^{1} \mathrm{H} \mathrm{NMR}\left(\mathrm{CDCl}_{3}\right) \delta 2.78$ (dd, $J=16.4$ and $\left.2.8 \mathrm{~Hz}, 1 \mathrm{H}\right)$, $2.99(\mathrm{dd}, J=16.4$ and $12.8 \mathrm{~Hz}, 1 \mathrm{H}), 3.83(\mathrm{~s}, 3 \mathrm{H}), 3.90(\mathrm{~s}, 3 \mathrm{H}), 5.39(\mathrm{dd}, J=13.2$ and $2.8 \mathrm{~Hz}, 1 \mathrm{H}), 6.10(\mathrm{~d}, 2.4 \mathrm{~Hz}$, $1 \mathrm{H}), 6.15(\mathrm{~d}, 2.4 \mathrm{~Hz}, 1 \mathrm{H}), 7.11(\mathrm{t}, J=8.8 \mathrm{~Hz}, 2 \mathrm{H}), 7.44(\mathrm{dd}, J=8.8$ and $5.2 \mathrm{~Hz}, 2 \mathrm{H}) ;{ }^{13} \mathrm{C} \mathrm{NMR}\left(\mathrm{CDCl}_{3}\right) \delta 45.5,55.6$, 56.1, 78.5, 93.2, 93.5, 105.9, 115.7 (d, $J=22.2 \mathrm{~Hz}), 127.9$ (d, $J=5.0 \mathrm{~Hz}), 134.6$ (d, $J=2.9 \mathrm{~Hz}), 162.3,162.7$ (d, $J$ $=245.7 \mathrm{~Hz}), 164.7,166.0,188.8$; HRMS $\left(\right.$ ESI-MS) $\mathrm{m} / \mathrm{z}$ calcd $\mathrm{C}_{17} \mathrm{H}_{15} \mathrm{FO}_{4}+\mathrm{H} 303.1027$, found 303.1023 .

2-(3-Chlorophenyl)-5,7-dimethoxychroman-4-one 4i. 79\% yield; colorless crystal; m.p. 148-149 ${ }^{\circ} \mathrm{C}$; IR ( $\left.\mathrm{cm}^{-1}, \mathrm{KBr}\right)$ 1657; ${ }^{1} \mathrm{H}$ NMR $\left(\mathrm{CDCl}_{3}\right) \delta 2.80(\mathrm{dd}, J=16.4$ and $2.9 \mathrm{~Hz}, 1 \mathrm{H}), 2.96(\mathrm{dd}, J=16.4$ and $12.8 \mathrm{~Hz}, 1 \mathrm{H}), 3.84(\mathrm{~s}, 3 \mathrm{H}), 3.90$ (s, 3H), 5.39 (dd, $J=13.0$ and $2.9 \mathrm{~Hz}, 1 \mathrm{H}), 6.11(\mathrm{~d}, J=2.4 \mathrm{~Hz}, 1 \mathrm{H}), 6.17(\mathrm{~d}, J=2.4 \mathrm{~Hz}, 1 \mathrm{H}), 7.30-7.35(\mathrm{~m}, 3 \mathrm{H})$, $7.49(\mathrm{~s}, 1 \mathrm{H}) ;{ }^{13} \mathrm{C} \mathrm{NMR}\left(\mathrm{CDCl}_{3}\right) \delta 45.5,55.6,56.1,78.3,93.3,93.5,105.9,124.0,126.2,128.7,130.0,134.7,140.8$, 162.2, 164.6, 166.0, 188.5; HRMS (ESI-MS) $\mathrm{m} / \mathrm{z}$ calcd for $\mathrm{C}_{17} \mathrm{H}_{15} \mathrm{ClO}_{4}+\mathrm{H} 319.0732$, found 319.0738.

2-(4-Chlorophenyl)-5,7-dimethoxychroman-4-one 4j. 78\% yield; colorless crystal; m.p. 168-169 ${ }^{\circ} \mathrm{C}$ (conglomerate), m.p. $154-155^{\circ} \mathrm{C}$ (racemic); IR ( $\left.\mathrm{cm}^{-1}, \mathrm{KBr}\right) 1676 ;{ }^{1} \mathrm{H}$ NMR $\left(\mathrm{CDCl}_{3}\right) \delta 2.79(\mathrm{dd}, J=16.6$ and $2.9 \mathrm{~Hz}, 1 \mathrm{H}), 2.97$ (dd, $J$ 
$=16.4$ and $13.2 \mathrm{~Hz}, 1 \mathrm{H}), 3.83(\mathrm{~s}, 3 \mathrm{H}), 3.90(\mathrm{~s}, 3 \mathrm{H}), 5.39(\mathrm{dd}, J=12.8$ and $2.9 \mathrm{~Hz}, 1 \mathrm{H}), 6.10(\mathrm{~d}, J=2.0 \mathrm{~Hz}, 1 \mathrm{H}), 6.15$ $(\mathrm{d}, J=2.0 \mathrm{~Hz}, 1 \mathrm{H}), 7.40$ (s, 4H); ${ }^{13} \mathrm{C}$ NMR $\left(\mathrm{CDCl}_{3}\right) \delta 45.5,55.6,56.2,78.4,93.3,93.5,105.9,127.5,128.9,134.4$, 137.3, 162.3, 164.7, 166.0, 188.7; HRMS (ESI-MS) $\mathrm{m} / \mathrm{z}$ calcd for $\mathrm{C}_{17} \mathrm{H}_{15} \mathrm{ClO}_{4}+\mathrm{H} 319.0732$, found 319.0739.

2-(4-Bromophenyl)-5,7-dimethoxychroman-4-one 4k. 66\% yield; colorless crystal; m.p. 169-170 ${ }^{\circ} \mathrm{C}$ (conglomerate), m.p. $165-166{ }^{\circ} \mathrm{C}$ (racemic); IR ( $\left.\mathrm{cm}^{-1}, \mathrm{KBr}\right) 1678 ;{ }^{1} \mathrm{H}$ NMR $\left(\mathrm{CDCl}_{3}\right) \delta 2.78(\mathrm{dd}, J=16.4$ and $3.0 \mathrm{~Hz}, 1 \mathrm{H}), 2.96(\mathrm{dd}, \mathrm{J}$ $=16.6$ and $13.0 \mathrm{~Hz}, 1 \mathrm{H}), 3.83(\mathrm{~s}, 3 \mathrm{H}), 3.89(\mathrm{~s}, 3 \mathrm{H}), 5.37(\mathrm{dd}, J=12.8$ and $3.0 \mathrm{~Hz}, 1 \mathrm{H}), 6.10(\mathrm{~d}, J=2.0 \mathrm{~Hz}, 1 \mathrm{H}), 6.15$ $(\mathrm{d}, J=2.4 \mathrm{~Hz}, 1 \mathrm{H}), 7.34(\mathrm{~d}, J=8.4 \mathrm{~Hz}, 2 \mathrm{H}), 7.54(\mathrm{~d}, J=8.4 \mathrm{~Hz}, 2 \mathrm{H}) ;{ }^{13} \mathrm{C}$ NMR $\left(\mathrm{CDCl}_{3}\right) \delta 45.4,55.6,56.1,78.4$, 93.2, 93.5, 105.9, 122.5, 127.7, 131.9, 137.8, 162.2, 164.6, 166.0, 188.6; HRMS (ESI-MS) $\mathrm{m} / \mathrm{z}$ calcd for $\mathrm{C}_{17} \mathrm{H}_{15} \mathrm{BrO}_{4}+\mathrm{H} 363.0226$, found 363.0236 .

5,7-Dimethoxy-2-(naphthalen-1-yl)chroman-4-one 4I. 76\% yield; colorless crystal; m.p. 157-158 ${ }^{\circ} \mathrm{C}$; (conglomerate), m.p. $152-153{ }^{\circ} \mathrm{C}$ (racemic); IR ( $\left.\mathrm{cm}^{-1}, \mathrm{KBr}\right) 1664 ;{ }^{1} \mathrm{H} \mathrm{NMR}\left(\mathrm{CDCl}_{3}\right) \delta 3.01$ (dd, $J=16.8$ and $\left.2.8 \mathrm{~Hz}, 1 \mathrm{H}\right), 3.20$ (dd, $J$ $=16.6$ and $13.0 \mathrm{~Hz}, 1 \mathrm{H}), 3.82(\mathrm{~s}, 3 \mathrm{H}), 3.92(\mathrm{~s}, 3 \mathrm{H}), 6.13-6.19(\mathrm{~m}, 3 \mathrm{H}), 7.50-7.57(\mathrm{~m}, 3 \mathrm{H}), 7.73(\mathrm{~d}, \mathrm{~J}=7.2 \mathrm{~Hz}, 1 \mathrm{H})$, $7.88(\mathrm{q}, J=6.9 \mathrm{~Hz}, 1 \mathrm{H}), 8.03(\mathrm{~d}, J=8.0 \mathrm{~Hz}, 1 \mathrm{H}) ;{ }^{13} \mathrm{C} \mathrm{NMR}\left(\mathrm{CDCl}_{3}\right) \delta 44.8,55.6,56.2,76.3,93.2,93.6,106.1,122.8$, 123.7, 125.3, 125.9, 126.6, 129.0, 129.2, 162.3, 165.1, 165.9, 189.4; HRMS (ESI-MS) $\mathrm{m} / \mathrm{z}$ calcd for $\mathrm{C}_{21} \mathrm{H}_{18} \mathrm{O}_{4}+\mathrm{H}$ 335.1278 , found 335.1283 .

5,7-Dimethoxy-2-(naphthalen-2-yl)chroman-4-one $4 \mathrm{~m}$. $75 \%$ yield; colorless crystal; m.p. $216-217{ }^{\circ} \mathrm{C}$ (conglomerate), m.p. 205-206 ${ }^{\circ} \mathrm{C}$ (racemic); IR ( $\left.\mathrm{cm}^{-1}, \mathrm{KBr}\right) 1669 ;{ }^{1} \mathrm{H}$ NMR $\left(\mathrm{CDCl}_{3}\right) \delta 2.88$ (dd, $J=16.6$ and $\left.3.0 \mathrm{~Hz}, 1 \mathrm{H}\right), 3.12$ (dd, $J=16.4$ and $13.2 \mathrm{~Hz}, 1 \mathrm{H}), 3.83(\mathrm{~s}, 3 \mathrm{H}), 3.91(\mathrm{~s}, 3 \mathrm{H}), 5.57(\mathrm{dd}, J=13.0$ and $3.0 \mathrm{~Hz}, 1 \mathrm{H}), 6.11(\mathrm{~d}, 2.4 \mathrm{~Hz}, 1 \mathrm{H})$, $6.20(\mathrm{~d}, 2.4 \mathrm{~Hz}, 1 \mathrm{H}), 7.51(\mathrm{~d}, J=9.2 \mathrm{~Hz}, 1 \mathrm{H}), 7.51(\mathrm{dd}, J=3.2$ and $1.2 \mathrm{~Hz}, 1 \mathrm{H}), 7.56(\mathrm{dd}, J=8.6$ and $1.8 \mathrm{~Hz}, 1 \mathrm{H})$, $7.86(\mathrm{q}, J=4.5 \mathrm{~Hz}, 2 \mathrm{H}), 7.9(\mathrm{~d}, \mathrm{~J}=9.2 \mathrm{~Hz}, 2 \mathrm{H}) ;{ }^{13} \mathrm{C} \mathrm{NMR}\left(\mathrm{CDCl}_{3}\right) \delta 45.6,55.6,56.1,79.3,93.2,93.5,106.0,123.6$, 125.3, 126.4, 127.7, 128.1, 128.7, 133.1, 133.3, 136.1, 162.256, 164.924, 165.952, 189.066; HRMS (ESI-MS) m/z calcd for $\mathrm{C}_{21} \mathrm{H}_{18} \mathrm{O}_{4}+\mathrm{H} 335.1278$, found 335.1283 . 


\section{Single crystal X-ray structure analysis of $\mathbf{4}$}

Single crystal $X$-ray structure analysis of $4 a$. Colorless plate, $\left(0.30 \times 0.20 \times 0.10 \mathrm{~mm}^{3}\right)$, orthorhombic space group $P 212121, a=5.442(3) \AA, b=8.871(5) \AA, c=28.748(16) \AA, V=1387.8(14) \AA^{3}, Z=4, \lambda(\mathrm{CuK} \alpha)=1.54178 \AA, \rho=$ $1.361 \mathrm{~g} / \mathrm{cm}^{3}, \mu(\mathrm{CuK} \alpha)=0.097 \mathrm{~mm}^{-1}, 6533$ reflections measured $\left(T=173 \mathrm{~K}, 1.417^{\circ}<\theta<27.426^{\circ}\right)$, nb of independent data collected: 2956, nb of independent data used for refinement: 2056 in the final least-squares refinement cycles on $\mathrm{F}^{2}$, the model converged at $R_{1}=0.0613, w R_{2}=0.1267[\mathrm{I}>2 \sigma(\mathrm{I})], R_{1}=0.1005, w R_{2}=0.1501$ (all data), and $\mathrm{GOF}=1.048, \mathrm{H}$-atom parameters constrained.

CCDC 2006511 (for 4a) contains the supplementary crystallographic data for this paper. These data can be obtained free of charge from The Cambridge Crystallographic Data Centre.

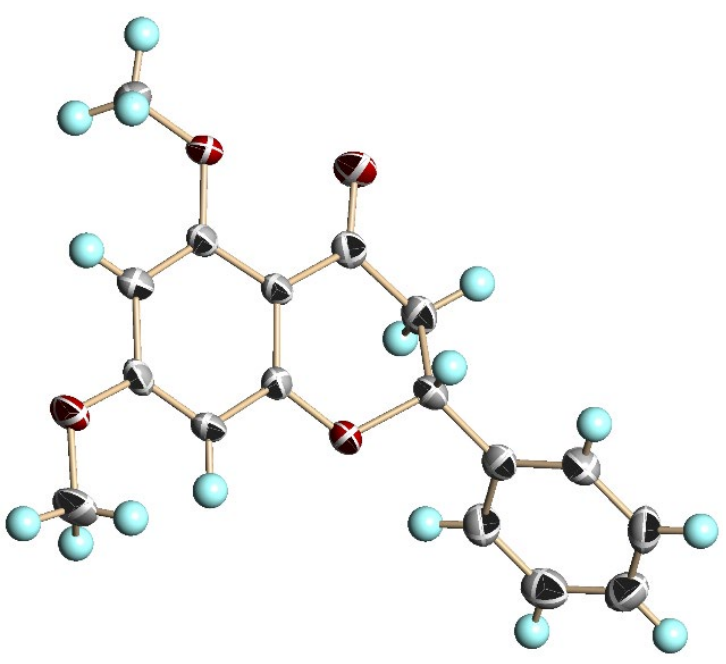

Figure S1. Perspective view of $(R)-\mathbf{4 a}$.

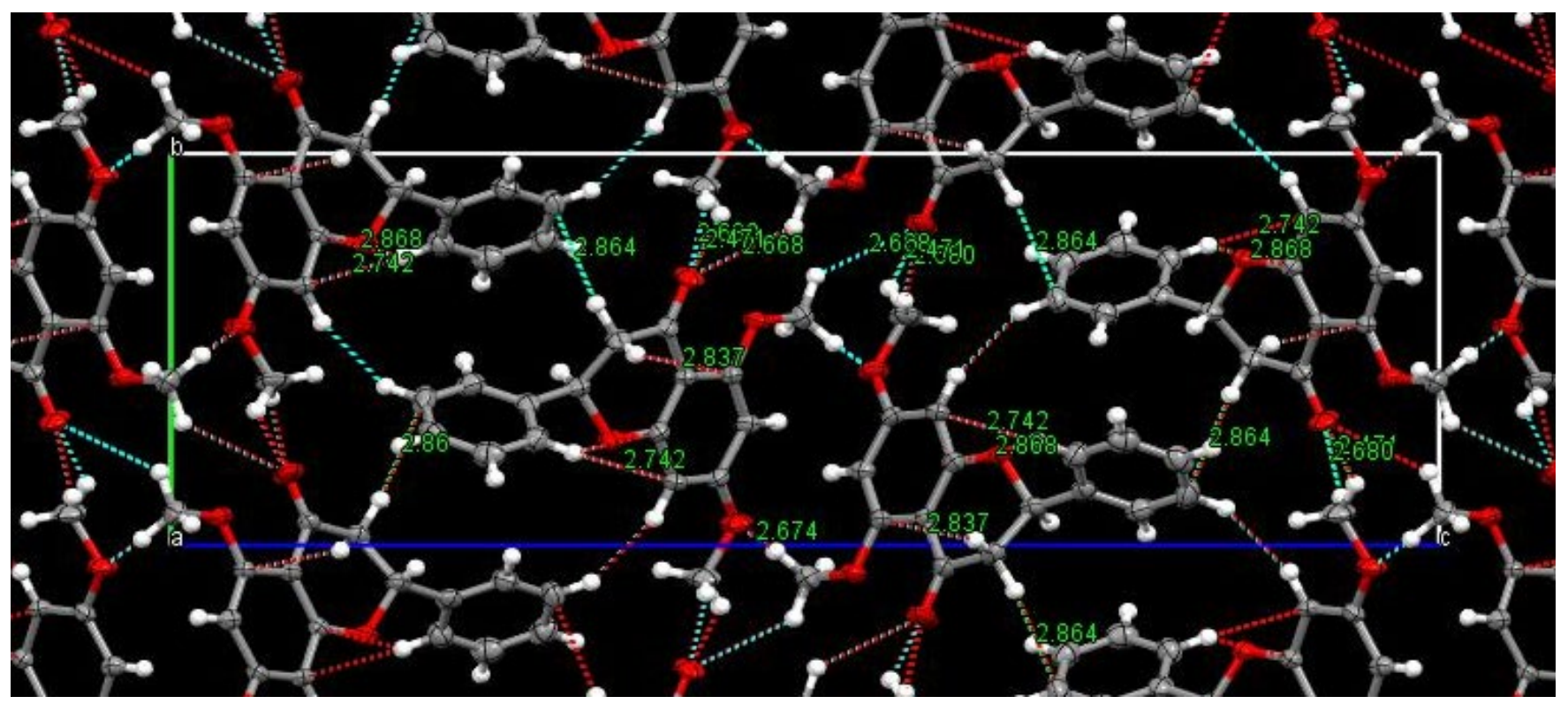

Figure S2. Packing diagram of $(R)-4 a$ along the a-axis. 
Single crystal $X$-ray structure analysis of $4 \mathbf{b}$. Colorless prism, $\left(0.200 \times 0.100 \times 0.050 \mathrm{~mm}^{3}\right)$, orthorhombic space group Pbca, $a=19.388(3) \AA, b=7.5303(13) \AA, c=20.341(3) \AA, V=2969.7(9) \AA^{3}, Z=8, \lambda($ MoK $\alpha)=0.71073 \AA, \rho$ $=1.334 \mathrm{~g} / \mathrm{cm}^{3}, \mu(\mathrm{MoK} \alpha)=0.094 \mathrm{~mm}^{-1}, 15858$ reflections measured $\left(T=173 \mathrm{~K}, 2.002^{\circ}<\theta<27.552^{\circ}\right)$, nb of independent data collected: 3392, $\mathrm{nb}$ of independent data used for refinement: 1889 in the final least-squares refinement cycles on $\mathrm{F}^{2}$, the model converged at $R_{1}=0.0596, w R_{2}=0.1537[\mathrm{I}>2 \sigma(\mathrm{I})], R_{1}=0.1178, w R_{2}=0.1871$ (all data), and GOF $=1.033, \mathrm{H}$-atom parameters constrained.

CCDC 1988302 (for $\mathbf{4 b}$ ) contains the supplementary crystallographic data for this paper. These data can be obtained free of charge from The Cambridge Crystallographic Data Centre.

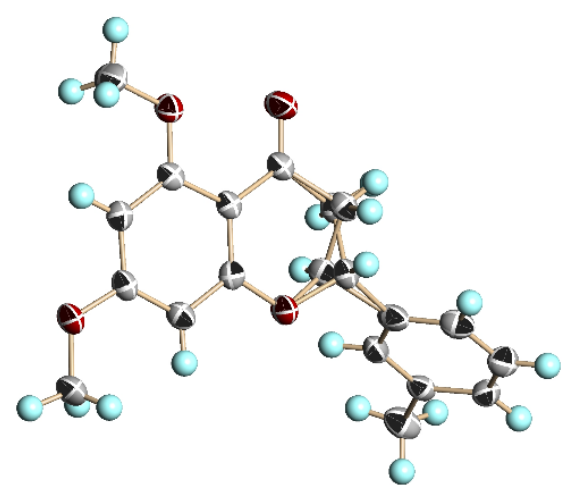

Figure S3. Perspective view of $\mathbf{4 b}$.

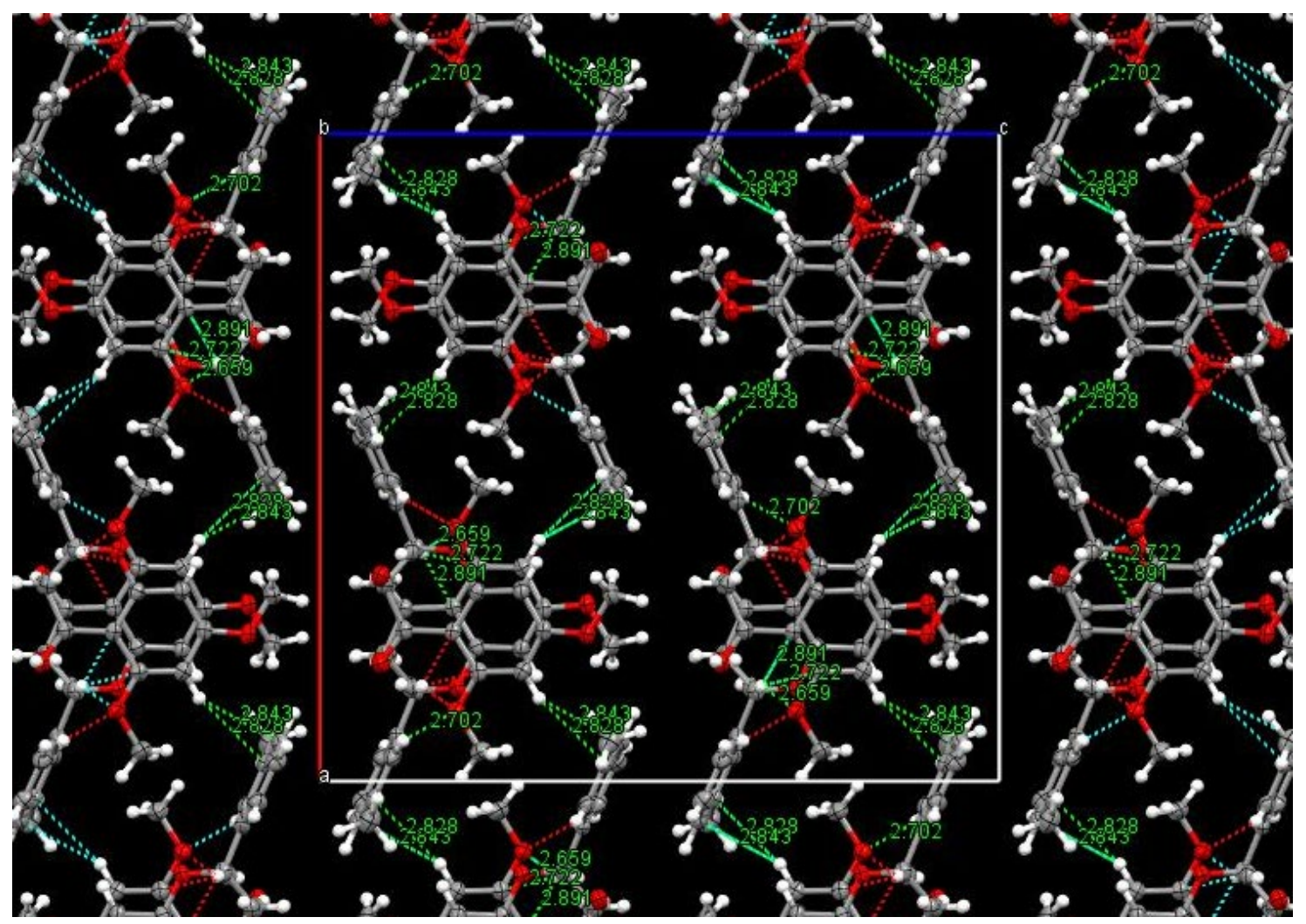

Figure S4. Packing diagram of $\mathbf{4 b}$ along the $b$-axis. 
Single crystal $X$-ray structure analysis of $4 c$. Colorless needle, $\left(0.300 \times 0.100 \times 0.050 \mathrm{~mm}^{3}\right)$, orthorhombic space group $P 2{ }_{1} 2121, a=5.6145(11) \AA, b=8.7362(18) \AA, c=30.511(6) \AA, V=1496.5(5) \AA^{3}, Z=4, \lambda($ MoK $\alpha)=0.71073$ $\AA, \rho=1.324 \mathrm{~g} / \mathrm{cm}^{3}, \mu(\mathrm{MoK} \alpha)=0.093 \mathrm{~mm}^{-1}, 8646$ reflections measured $\left(T=173 \mathrm{~K}, 1.335^{\circ}<\theta<27.557^{\circ}\right), \mathrm{nb}$ of independent data collected: 3362, nb of independent data used for refinement: 1701 in the final least-squares refinement cycles on $\mathrm{F}^{2}$, the model converged at $R_{1}=0.0587, w R_{2}=0.1191[\mathrm{I}>2 \sigma(\mathrm{I})], R_{1}=0.1553, w R_{2}=0.1714$ (all data), and GOF $=0.976, \mathrm{H}$-atom parameters constrained.

CCDC 2006512 (for 4c) contains the supplementary crystallographic data for this paper. These data can be obtained free of charge from The Cambridge Crystallographic Data Centre.

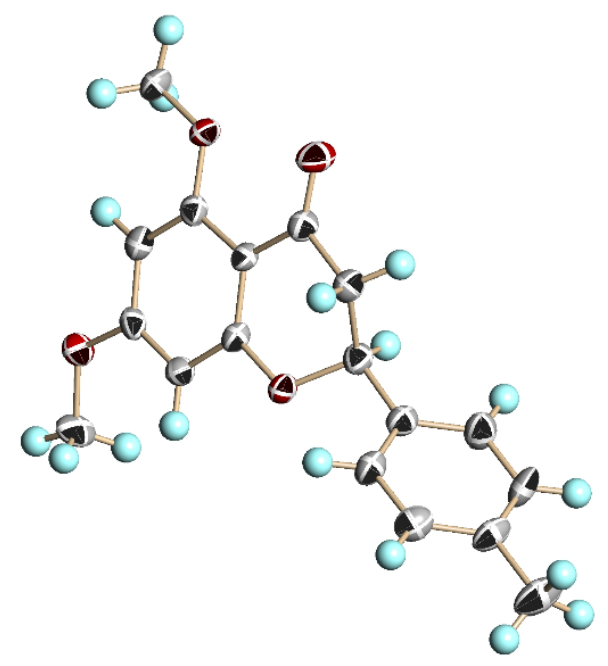

Figure S5. Perspective view of (S)-4c.

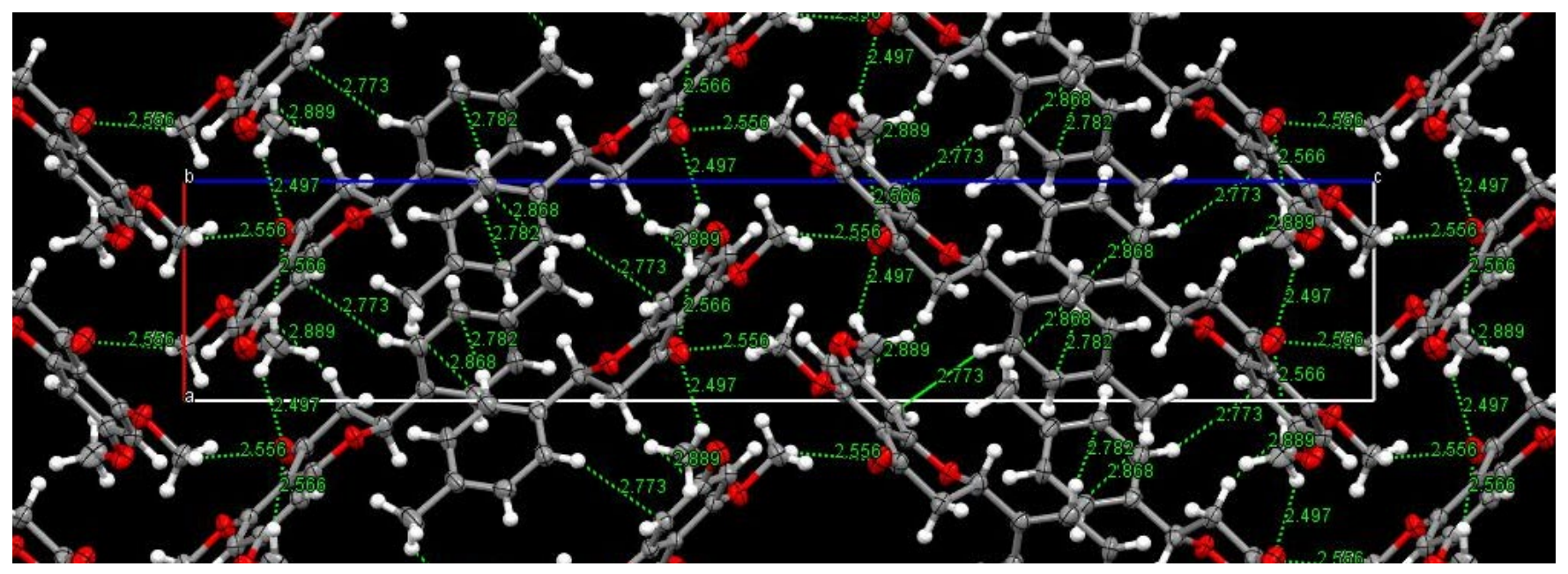

Figure S6. Perspective view of (S)-4c along the $b$-axis. 
Single crystal $X$-ray structure analysis of $\mathbf{4 d}$. Colorless prism, $\left(0.500 \times 0.500 \times 0.200 \mathrm{~mm}^{3}\right)$, monoclinic space group $P 2{ }_{1} / \mathrm{c}, a=16.7379(14) \AA, b=5.3110(5) \AA, c=17.5703(16) \AA, \beta=96.8360(10)^{\circ}, V=1550.8(2) \AA^{3}, Z=4, \lambda$ $(\mathrm{MoK} \alpha)=0.71073 \AA, \rho=1.346 \mathrm{~g} / \mathrm{cm}^{3}, \mu(\mathrm{MoK} \alpha)=0.098 \mathrm{~mm}^{-1}, 8511$ reflections measured $\left(T=173 \mathrm{~K}, 1.225^{\circ}<\theta\right.$ $\left.<27.575^{\circ}\right)$, $\mathrm{nb}$ of independent data collected: $3541, \mathrm{nb}$ of independent data used for refinement: 2369 in the final least-squares refinement cycles on $\mathrm{F}^{2}$, the model converged at $R_{1}=0.0448, w R_{2}=0.1213[\mathrm{I}>2 \sigma(\mathrm{I})], R_{1}=0.0734$, $w R_{2}=0.1464$ (all data), and GOF $=1.034, \mathrm{H}$-atom parameters constrained.

CCDC 1988303 (for 4d) contains the supplementary crystallographic data for this paper. These data can be obtained free of charge from The Cambridge Crystallographic Data Centre.

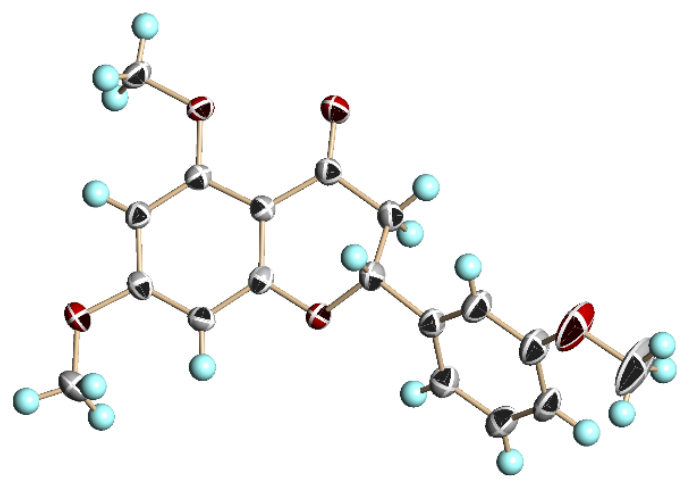

Figure S7. Perspective view of $\mathbf{4 d .}$

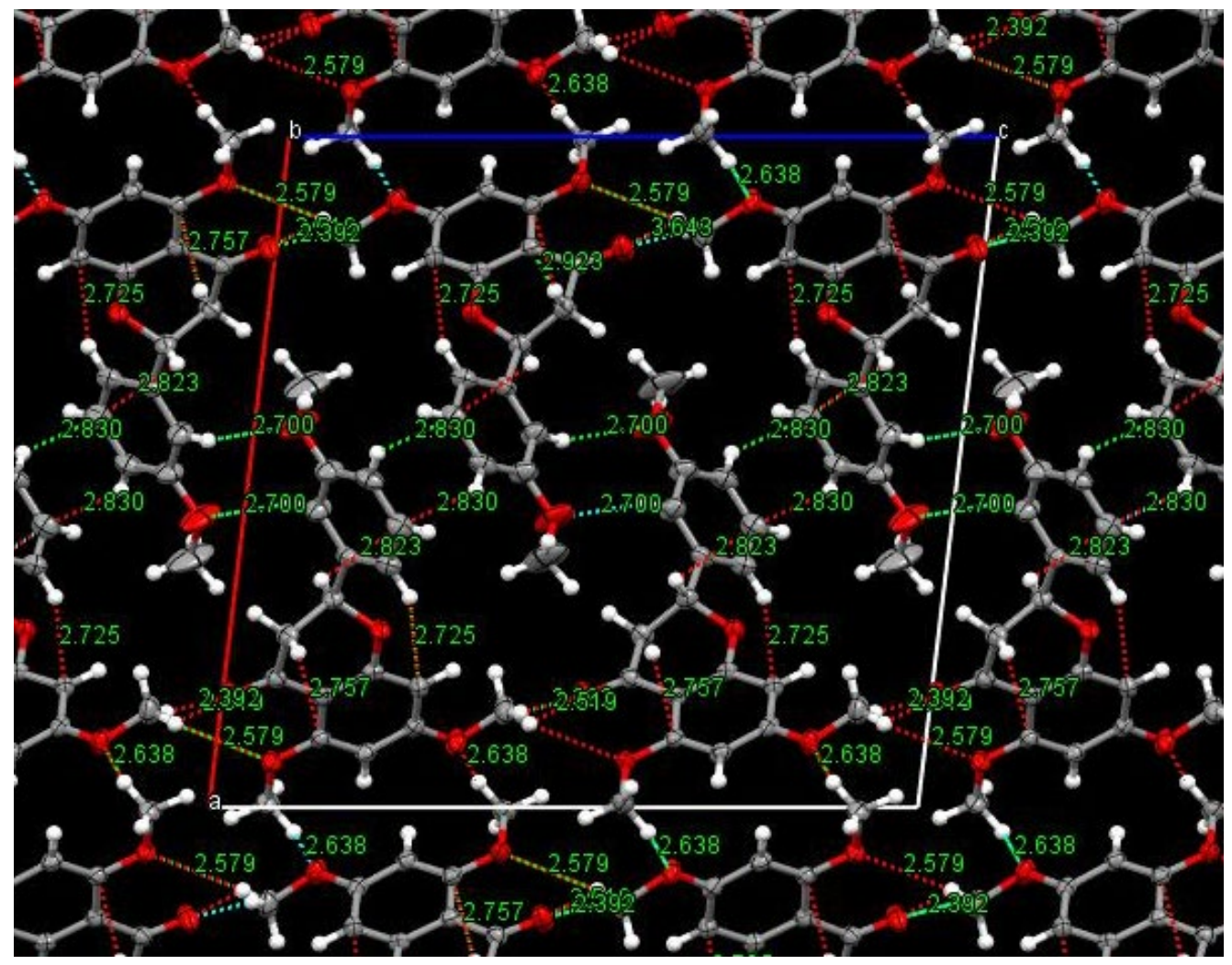

Figure S8. Packing diagram of $\mathbf{4 d}$ along the $b$-axis. 
Single crystal $X$-ray structure analysis of $4 \mathbf{e}$. Colorless needle, $\left(0.50 \times 0.10 \times 0.05 \mathrm{~mm}^{3}\right)$, monoclinic space group $P 21 / c, a=15.141(4) \AA, b=4.4681(11) \AA, c=23.386(6) \AA, \beta=105.385(3)^{\circ}, V=1525.4(6) \AA^{3}, Z=4, \lambda(\mathrm{MoK} \alpha)=$ $0.71073 \AA, \rho=1.346 \mathrm{~g} / \mathrm{cm}^{3}, \mu(\mathrm{MoK} \alpha)=0.100 \mathrm{~mm}^{-1}, 8192$ reflections measured $\left(T=173 \mathrm{~K}, 1.395^{\circ}<\theta<27.461\right.$ $\left.{ }^{\circ}\right)$, nb of independent data collected: 3420 , nb of independent data used for refinement: 1445 in the final leastsquares refinement cycles on $\mathrm{F}^{2}$, the model converged at $R_{1}=0.0642, w R_{2}=0.1553[\mathrm{I}>2 \sigma(\mathrm{I})], R_{1}=0.1734, w R_{2}$ $=0.2239$ (all data), and GOF $=0.967, \mathrm{H}$-atom parameters constrained.

CCDC 1988304 (for 4e) contains the supplementary crystallographic data for this paper. These data can be obtained free of charge from The Cambridge Crystallographic Data Centre.

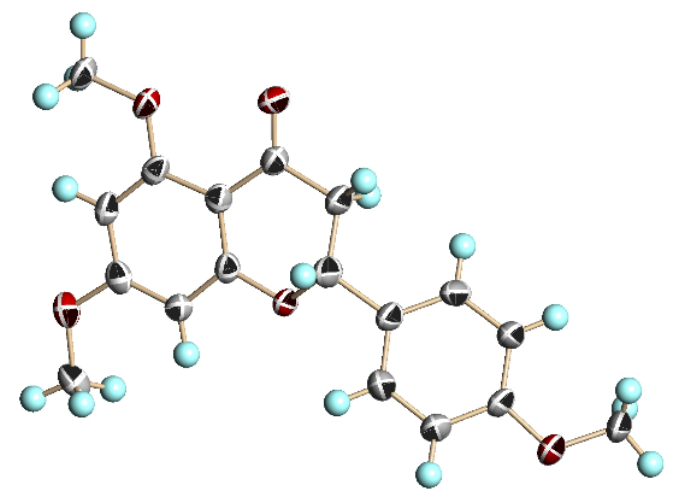

Figure S9. Perspective view of $4 \mathbf{e}$.

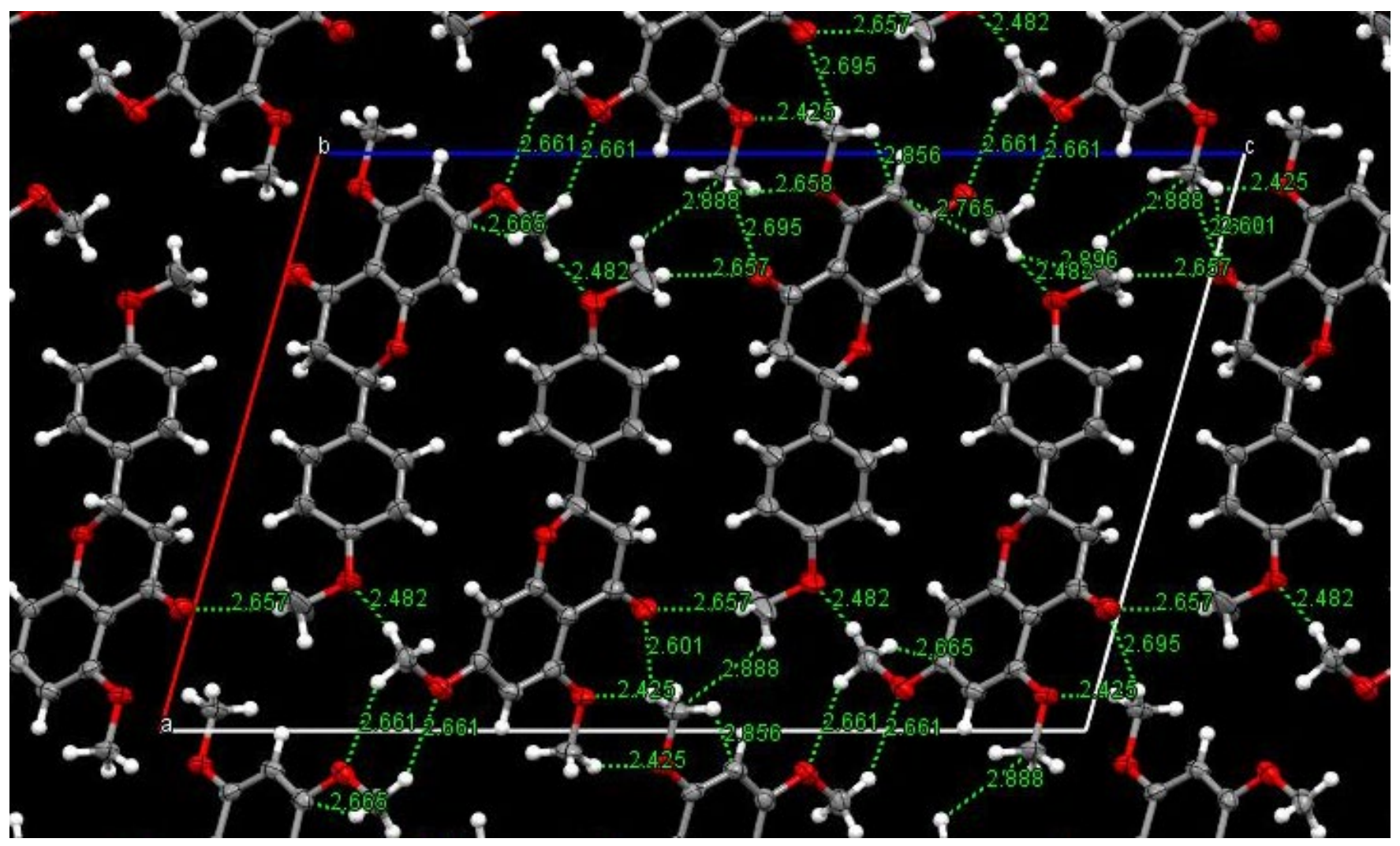

Figure S10. Packing diagram of $4 \mathrm{e}$ along the $b$-axis. 
Single crystal $X$-ray structure analysis of $\mathbf{4 f}$. Colorless prism, $\left(0.50 \times 0.50 \times 0.20 \mathrm{~mm}^{3}\right)$, monoclinic space group $P 21 / \mathrm{c}, a=12.3817(15) \AA, b=15.8883(19) \AA, c=8.6053(11) \AA, \beta=102.902(2)^{\circ}, V=1650.1(4) \AA^{3}, Z=4, \lambda(\mathrm{MoK} \alpha)$ $=0.71073 \AA, \rho=1.386 \mathrm{~g} / \mathrm{cm}^{3}, \mu(\mathrm{MoK} \alpha)=0.103 \mathrm{~mm}^{-1}, 9336$ reflections measured $\left(T=173 \mathrm{~K}, 1.687^{\circ}<\theta<27.448\right.$ $\left.{ }^{\circ}\right)$, nb of independent data collected: 3747 , nb of independent data used for refinement: 2751 in the final leastsquares refinement cycles on $\mathrm{F}^{2}$, the model converged at $R_{1}=0.0470, w R_{2}=0.1034[\mathrm{I}>2 \sigma(\mathrm{I})], R_{1}=0.0670, w R_{2}$ $=0.1171$ (all data), and GOF = 1.048, $\mathrm{H}$-atom parameters constrained.

CCDC 1988305 (for $\mathbf{4 f}$ ) contains the supplementary crystallographic data for this paper. These data can be obtained free of charge from The Cambridge Crystallographic Data Centre.

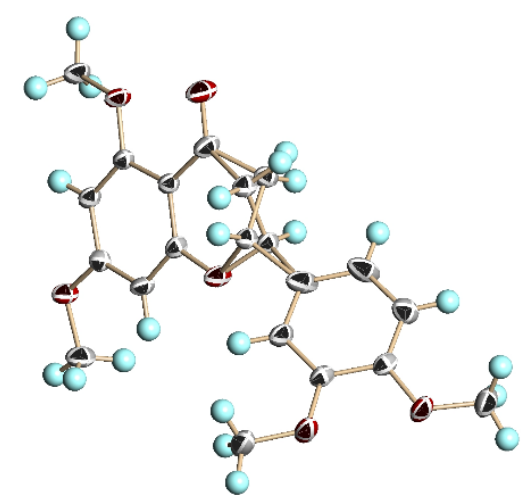

Figure S11. Perspective view of $\mathbf{4 f}$.

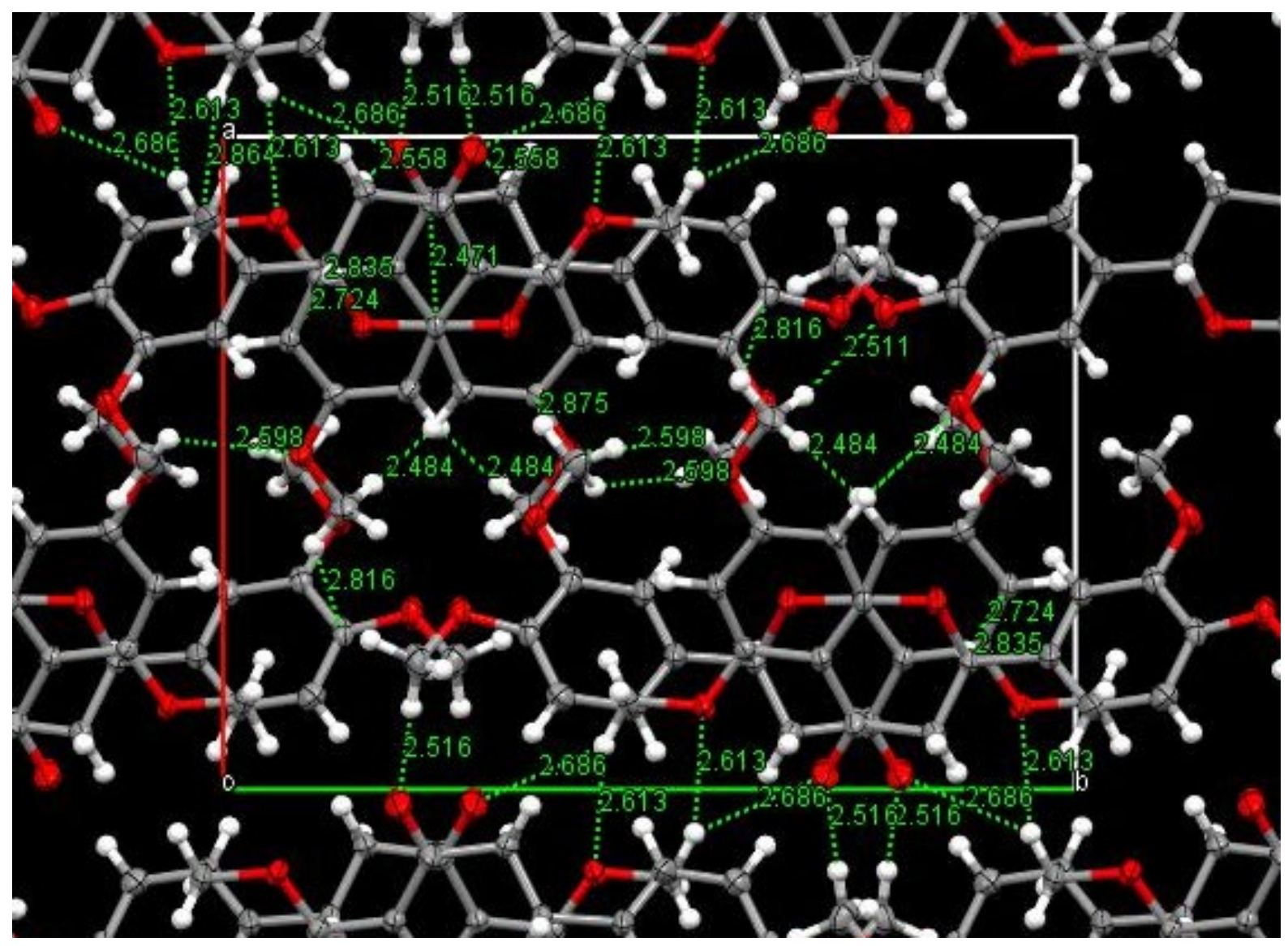

Figure S12. Packing diagram of $\mathbf{4 f}$ along the $c$-axis. 
Single crystal $X$-ray structure analysis of $\mathbf{4 g}$. Colorless prism, $\left(0.500 \times 0.500 \times 0.300 \mathrm{~mm}^{3}\right)$, triclinic space group $P$ $1, a=7.715(2) \AA, b=10.212(3) \AA, c=11.688(4) \AA, \alpha=75.765(4)^{\circ}, \beta=73.429(4)^{\circ}, \gamma=73.145(4)^{\circ}, V=831.2(4)$ $\mathrm{A}^{3}, Z=2, \lambda(\operatorname{MoK} \alpha)=0.71073 \AA, \rho=1.376 \mathrm{~g} / \mathrm{cm}^{3}, \mu(\operatorname{MoK} \alpha)=0.103 \mathrm{~mm}^{-1}, 4656$ reflections measured $(T=173 \mathrm{~K}$, $\left.1.847^{\circ}<\theta<27.533^{\circ}\right), \mathrm{nb}$ of independent data collected: 3597, $\mathrm{nb}$ of independent data used for refinement: 2458 in the final least-squares refinement cycles on $\mathrm{F}^{2}$, the model converged at $R_{1}=0.0590, w R_{2}=0.1483[\mathrm{I}>2 \sigma(\mathrm{I})], R_{1}$ $=0.0916, w R_{2}=0.1680$ (all data), and GOF $=1.219, \mathrm{H}$-atom parameters constrained.

CCDC 1988306 (for $\mathbf{4 g}$ ) contains the supplementary crystallographic data for this paper. These data can be obtained free of charge from The Cambridge Crystallographic Data Centre.

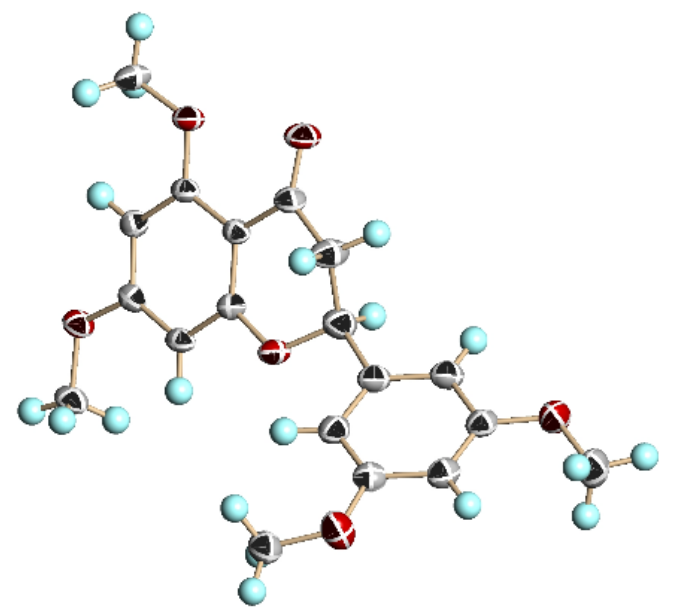

Figure S13. Perspective view of $\mathbf{4 g}$.

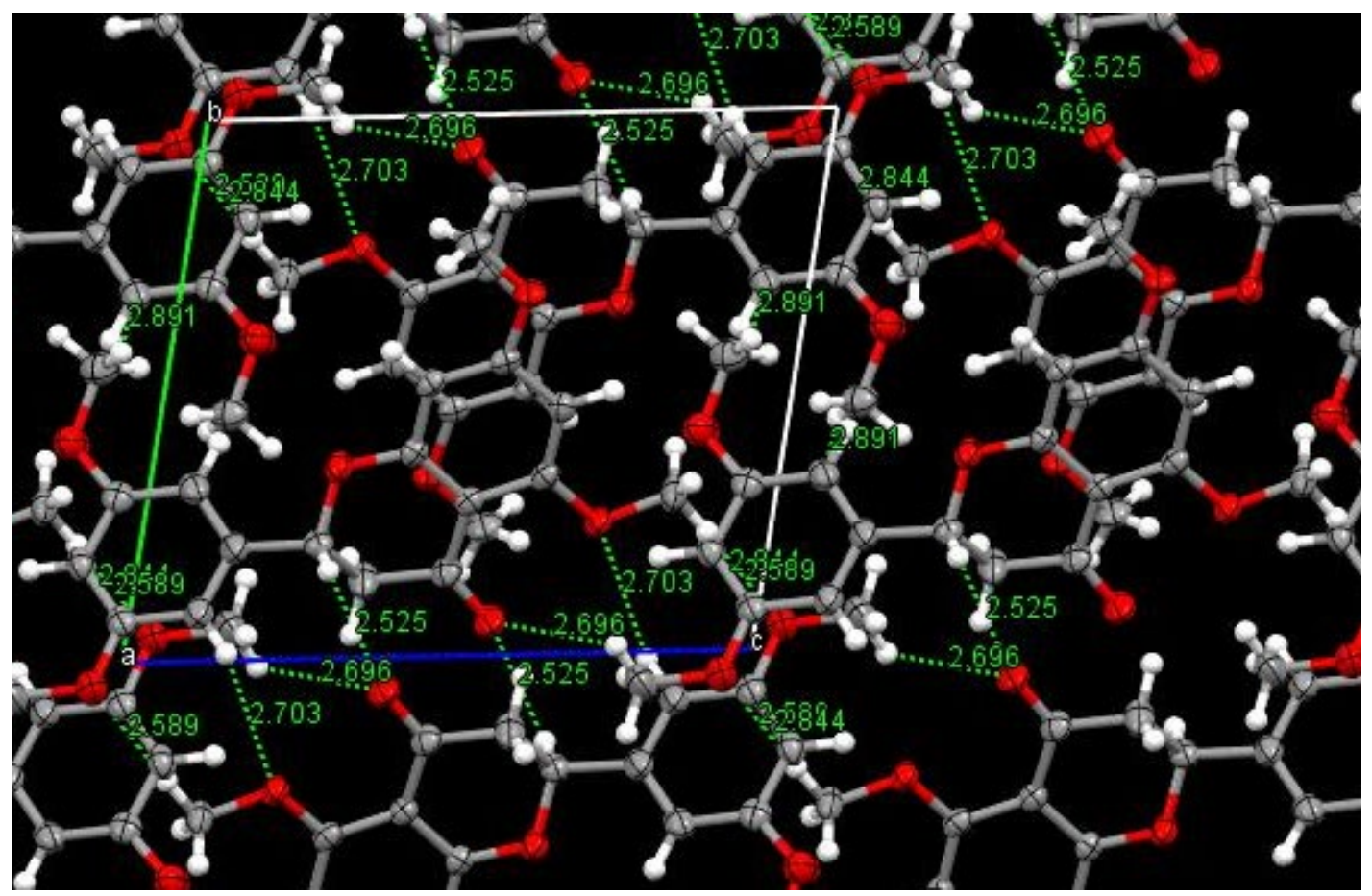

Figure S14. Packing diagram of $\mathbf{4 g}$ along the a-axis. 
Single crystal $X$-ray structure analysis of $\mathbf{4 h}$. Colorless plate, $\left(0.050 \times 0.030 \times 0.010 \mathrm{~mm}^{3}\right)$, orthorhombic space group $P 212121, a=5.4955(5) \AA, b=8.8034(6) \AA, c=29.525(2) \AA, V=1428.39(19) \AA^{3}, Z=4, \lambda(\mathrm{CuK} \alpha)=1.54178$ $\AA, \rho=1.406 \mathrm{~g} / \mathrm{cm}^{3}, \mu(\mathrm{CuK} \alpha)=0.911 \mathrm{~mm}^{-1}, 2397$ reflections measured $\left(T=173 \mathrm{~K}, 2.993^{\circ}<\theta<68.540^{\circ}\right)$, nb of independent data collected: $3846, \mathrm{nb}$ of independent data used for refinement: 1737 in the final least-squares refinement cycles on $\mathrm{F}^{2}$, the model converged at $R_{1}=0.0524, w R_{2}=0.1122[\mathrm{I}>2 \sigma(\mathrm{I})], R_{1}=0.0789, w R_{2}=0.1225$ (all data), and GOF $=1.038, \mathrm{H}$-atom parameters constrained.

CCDC 2006513 (for $\mathbf{4 h}$ ) contains the supplementary crystallographic data for this paper. These data can be obtained free of charge from The Cambridge Crystallographic Data Centre.

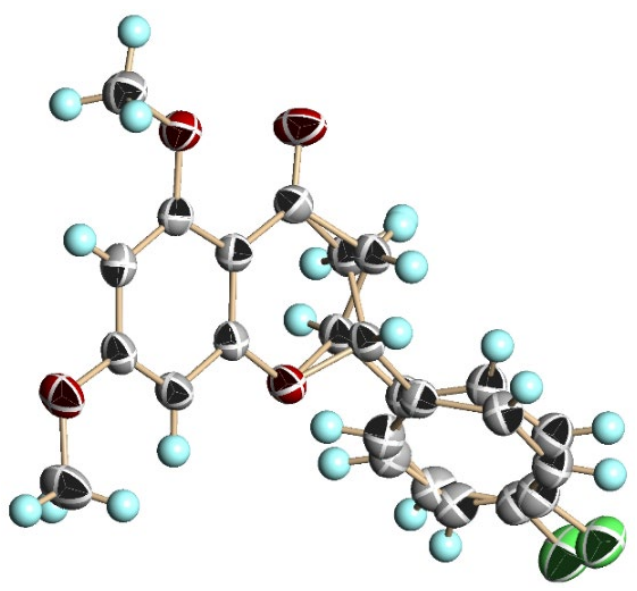

Figure S15. Perspective view of $\mathbf{4 h}$.

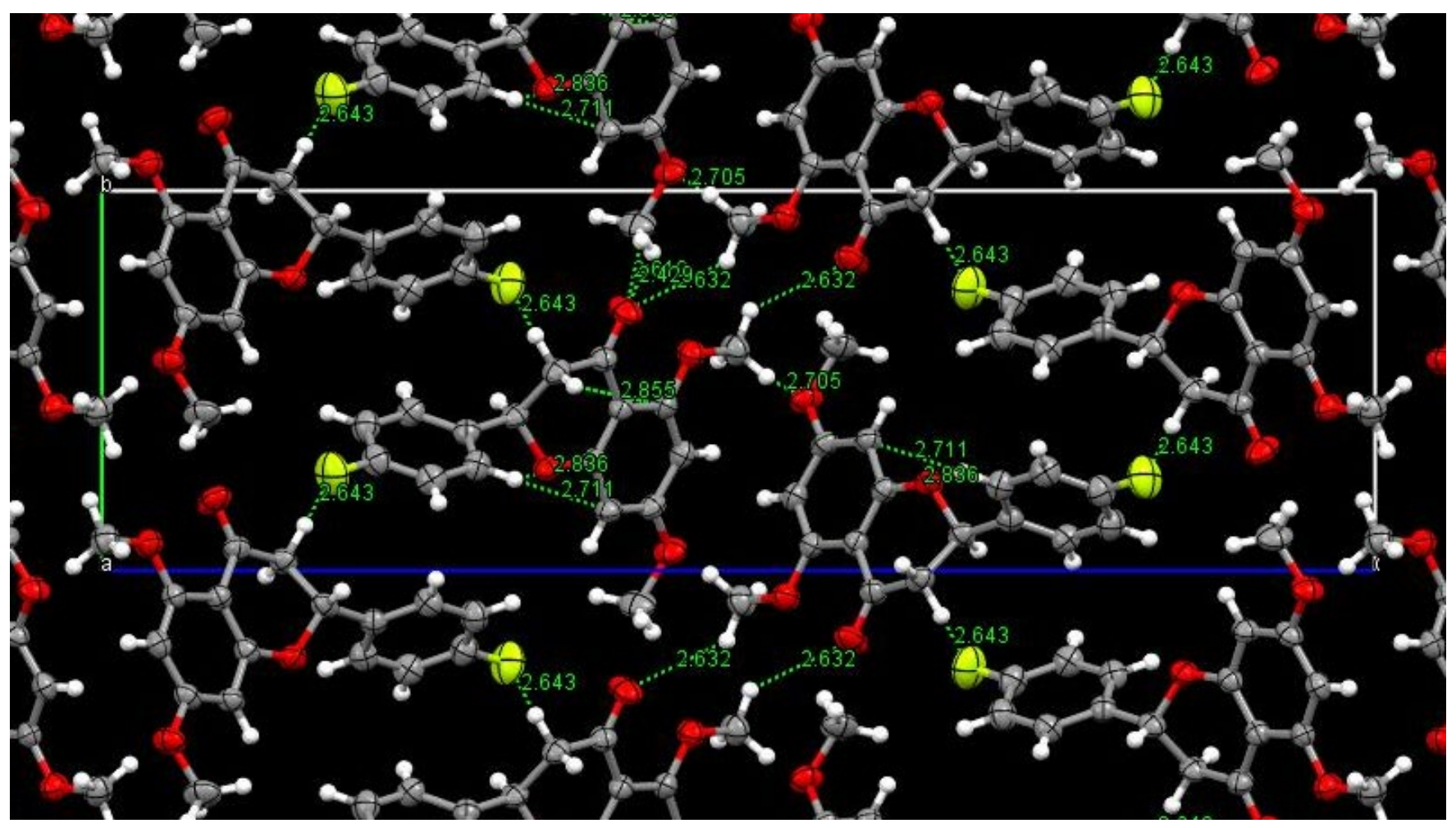

Figure S16. Packing diagram of $4 \mathrm{~h}$ along the a-axis. 
Single crystal $X$-ray structure analysis of 4 i. Colorless needle $\left(0.20 \times 0.02 \times 0.02 \mathrm{~mm}^{3}\right)$, orthorhombic space group Pbca, $a=19.3462(15) \AA, b=7.4904(6) \AA, c=20.3073(16) \AA, V=2942.7(4) \AA^{3}, Z=8, \lambda(\mathrm{CuK} \alpha)=1.54178 \AA, \rho=$ $1.439 \mathrm{~g} / \mathrm{cm}^{3}, \mu(\mathrm{CuK \alpha})=0.911 \mathrm{~mm}^{-1}, 18294$ reflections measured $\left(T=173 \mathrm{~K}, 4.354{ }^{\circ}<\theta<68.222^{\circ}\right)$, nb of independent data collected: $2660, \mathrm{nb}$ of independent data used for refinement: 1892 in the final least-squares refinement cycles on $\mathrm{F}^{2}$, the model converged at $R_{1}=0.0542, w R_{2}=0.1266[\mathrm{I}>2 \sigma(\mathrm{I})], R_{1}=0.0828, w R_{2}=0.1412$ (all data), and GOF $=1.031, \mathrm{H}$-atom parameters constrained.

CCDC 1988318 (for 4i) contains the supplementary crystallographic data for this paper. These data can be obtained free of charge from The Cambridge Crystallographic Data Centre.

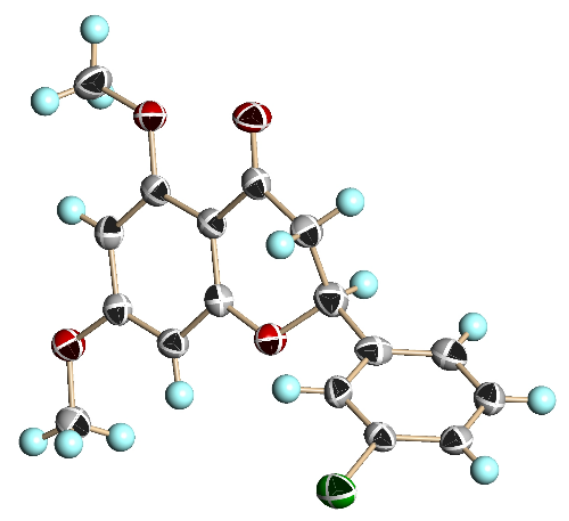

Figure S17. Perspective view of $\mathbf{4 i}$.

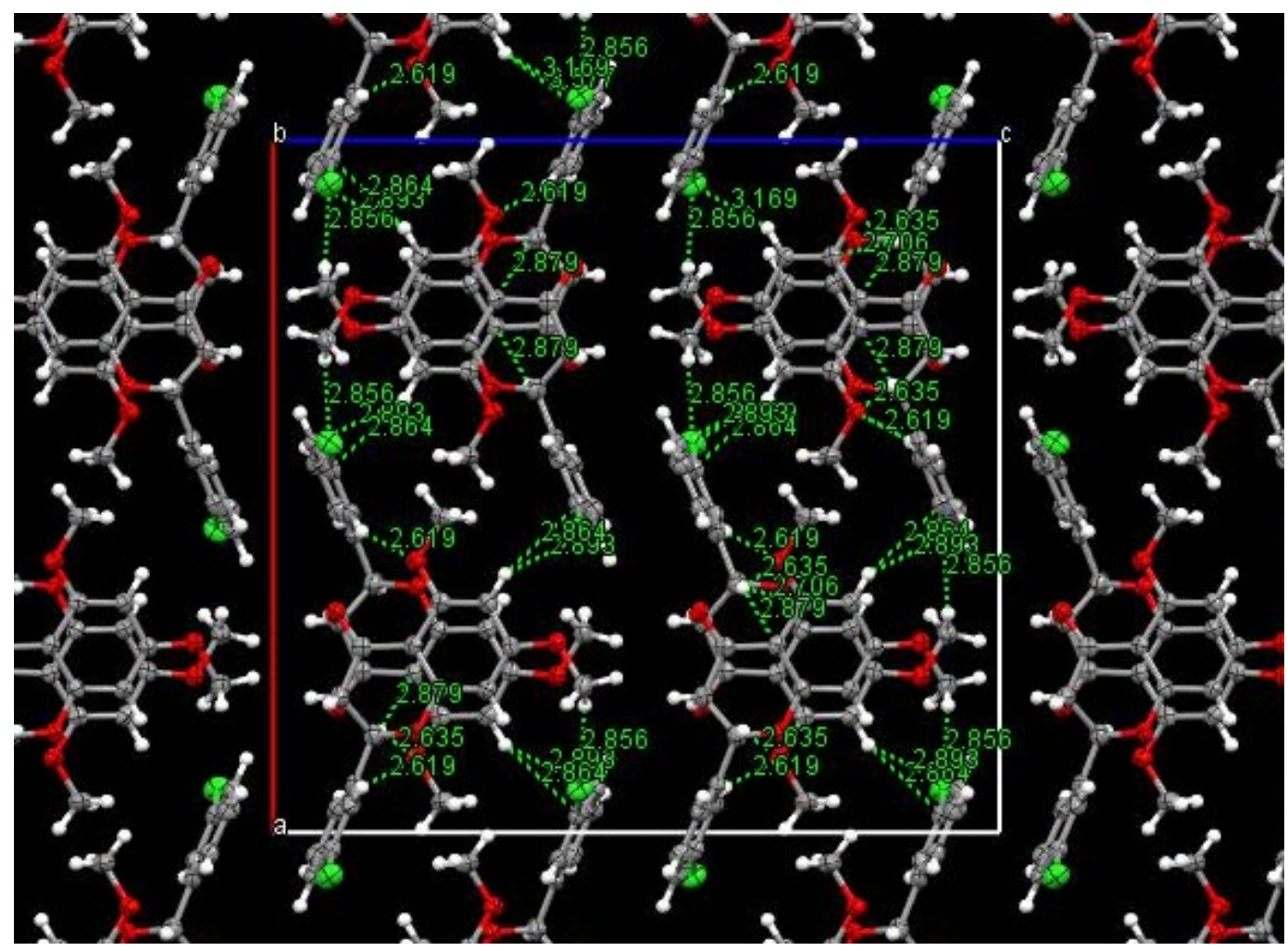

Figure S18. Packing diagram of $\mathbf{4 i}$ along the $b$-axis. 
Single crystal $X$-ray structure analysis of $4 j$. Colorless prism, $\left(0.500 \times 0.300 \times 0.300 \mathrm{~mm}^{3}\right)$, orthorhombic space group $P 2{ }_{12}{ }_{12}{ }_{1}, a=5.521(4) \AA, b=8.801(6) \AA, c=30.85(2) \AA, V=1499.0(18) \AA^{3}, Z=4, \lambda(\mathrm{CuK} \alpha)=1.54178 \AA, \rho$ $=1.412 \mathrm{~g} / \mathrm{cm}^{3}, \mu(\mathrm{CuK} \alpha)=0.270 \mathrm{~mm}^{-1}, 8609$ reflections measured $\left(T=173 \mathrm{~K}, 2.4065^{\circ}<\theta<27.590^{\circ}\right)$, nb of independent data collected: $3405, \mathrm{nb}$ of independent data used for refinement: 2875 in the final least-squares refinement cycles on $\mathrm{F}^{2}$, the model converged at $R_{1}=0.0376, w R_{2}=0.0833[\mathrm{I}>2 \sigma(\mathrm{I})], R_{1}=0.0472, w R_{2}=0.0876$ (all data), and GOF $=1.071, \mathrm{H}$-atom parameters constrained.

CCDC 2006515 (for $\mathbf{4 j}$ ) contains the supplementary crystallographic data for this paper. These data can be obtained free of charge from The Cambridge Crystallographic Data Centre.

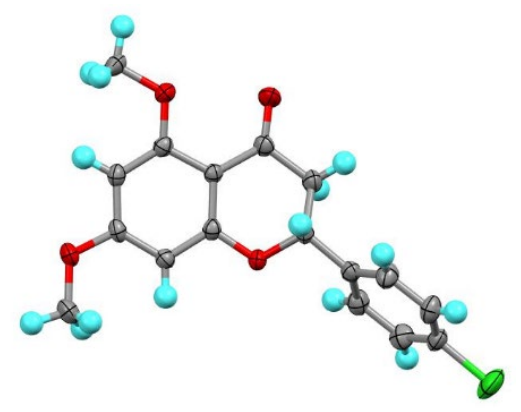

Figure S19. Perspective view of $(R)-\mathbf{4 j}$.

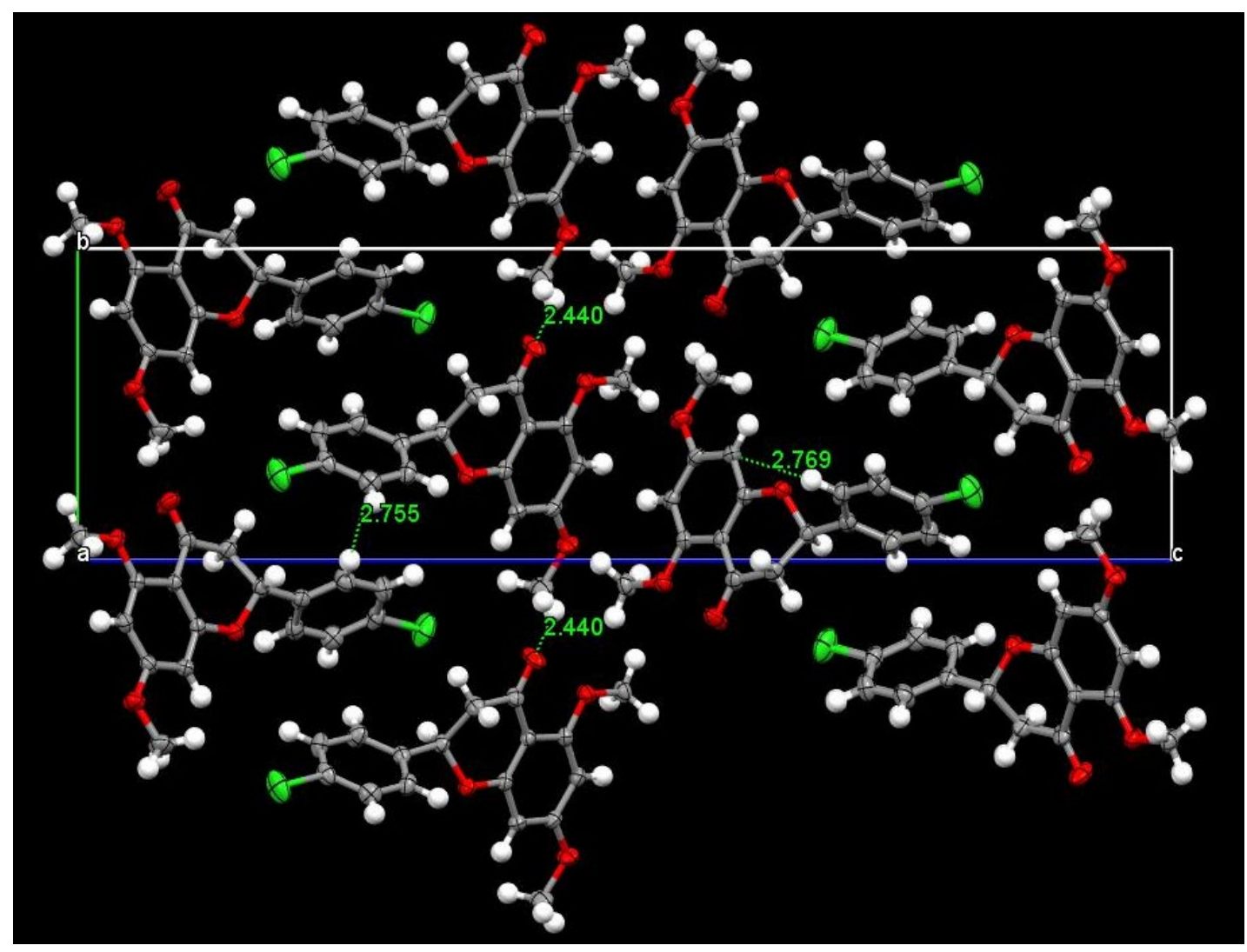

Figure S20. Packing diagram of $(R)-4 \mathbf{j}$ along the a-axis. 
Single crystal $X$-ray structure analysis of $\mathbf{4 k}$. Colorless prism, $\left(0.300 \times 0.100 \times 0.100 \mathrm{~mm}^{3}\right)$, orthorhombic space group $P 2{ }_{12} 22_{1}, a=5.4469(9) \AA, b=8.8456(13) \AA, c=31.639(5) \AA, V=1524.4(4) \AA^{3}, Z=4, \lambda(\mathrm{CuK} \alpha)=1.54178 \AA$, $\rho=1.583 \mathrm{~g} / \mathrm{cm}^{3}, \mu($ CuK $\alpha)=2.712 \mathrm{~mm}^{-1}, 8850$ reflections measured $\left(T=173 \mathrm{~K}, 1.287^{\circ}<\theta<27.530^{\circ}\right)$, nb of independent data collected: $3424, \mathrm{nb}$ of independent data used for refinement: 2626 in the final least-squares refinement cycles on $\mathrm{F}^{2}$, the model converged at $R_{1}=0.0466, w R_{2}=0.1183[\mathrm{I}>2 \sigma(\mathrm{I})], R_{1}=0.0683, w R_{2}=0.1361$ (all data), and GOF $=1.094, \mathrm{H}$-atom parameters constrained.

CCDC 2006516 (for $\mathbf{4 k}$ ) contains the supplementary crystallographic data for this paper. These data can be obtained free of charge from The Cambridge Crystallographic Data Centre.

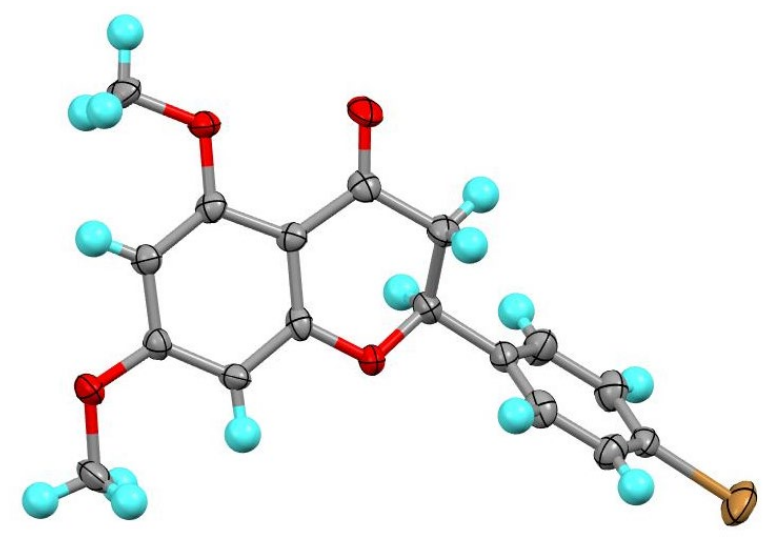

Figure S21. Perspective view of (S)-4k.

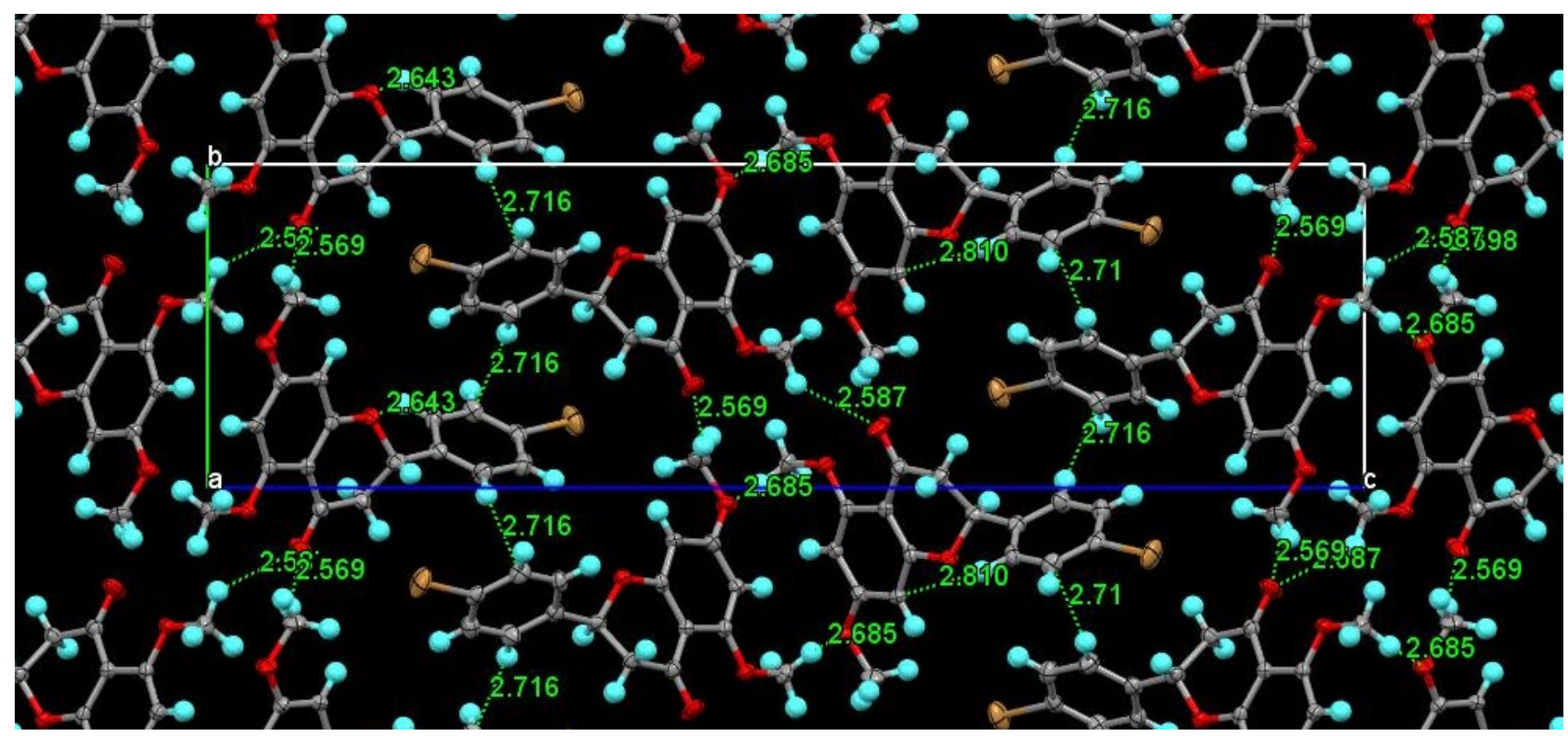

Figure S22. Packing diagram of (S)-4k along the a-axis. 
Single crystal $X$-ray structure analysis of $4 I$. Colorless plate $\left(0.200 \times 0.100 \times 0.020 \mathrm{~mm}^{3}\right)$, monoclinic space group $P 21, a=8.8108(7) \AA, b=5.9955(4) \AA, c=15.7023(13) \AA, \beta=97.468(6)^{\circ}, V=822.44(11) \AA^{3}, Z=2, \lambda(C u K \alpha)=$ $1.54178 \AA, \rho=1.350 \mathrm{~g} / \mathrm{cm}^{3}, \mu(\mathrm{CuK} \alpha)=0.758 \mathrm{~mm}^{-1}, 5415$ reflections measured $\left(\mathrm{T}=173 \mathrm{~K}, 2.838^{\circ}<\theta<68.234^{\circ}\right)$, $\mathrm{nb}$ of independent data collected: $2575, \mathrm{nb}$ of independent data used for refinement: 1812 in the final least-squares refinement cycles on $\mathrm{F}^{2}$, the model converged at $R_{1}=0.0552, w R_{2}=0.1313[\mathrm{I}>2 \sigma(\mathrm{I})], R_{1}=0.0861, w R_{2}=0.1472$ (all data), and GOF $=1.009, \mathrm{H}$-atom parameters constrained.

CCDC 2006517 (for 4I) contains the supplementary crystallographic data for this paper. These data can be obtained free of charge from The Cambridge Crystallographic Data Centre.

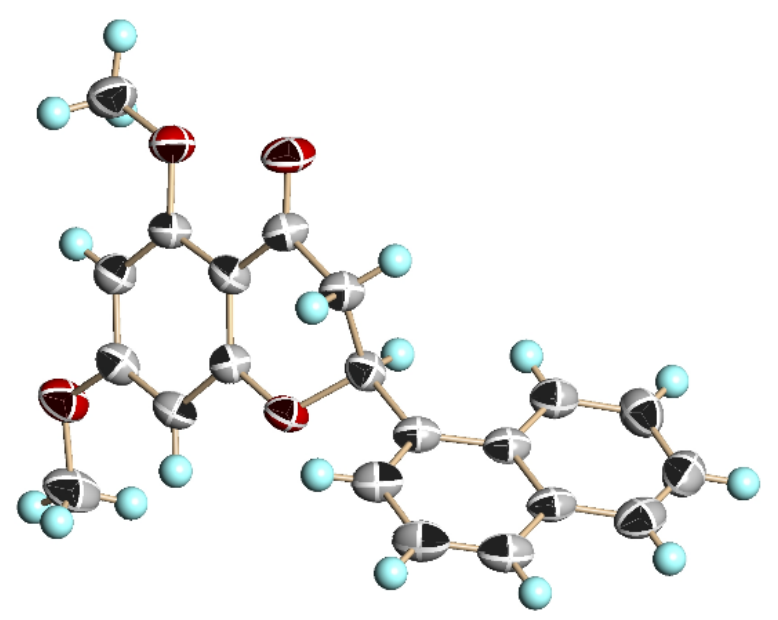

Figure S23. Perspective view of (S)-4I.

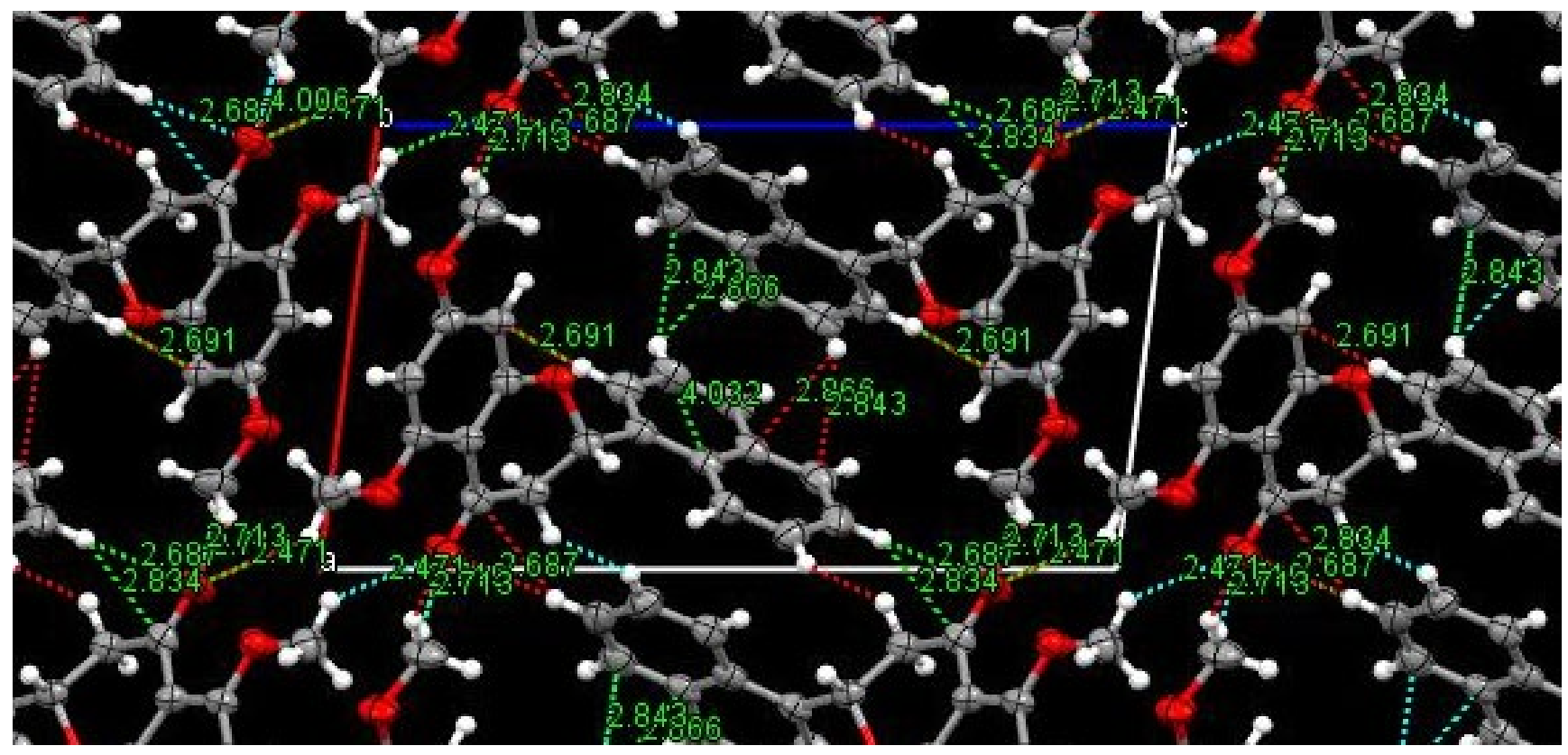

Figure S24. Packing diagram of (S)-4I along the $b$-axis. 
Single crystal $X$-ray structure analysis of $4 \mathrm{~m}$. Colorless needles $\left(0.200 \times 0.050 \times 0.030 \mathrm{~mm}^{3}\right)$, orthorhombic space group $P 2{ }_{12} 22_{1}, a=5.5067(3) \AA, b=8.7964(6) \AA, c=33.354(2) \AA, V=1615.64(17) \AA^{3}, Z=4, \lambda(\mathrm{CuK \alpha})=1.54178$ $\AA, \rho=1.375 \mathrm{~g} / \mathrm{cm}^{3}, \mu(\mathrm{CuK} \alpha)=0.771 \mathrm{~mm}^{-1}, 22255$ reflections measured $\left(\mathrm{T}=173 \mathrm{~K}, 2.649^{\circ}<\theta<68.595^{\circ}\right), \mathrm{nb}$ of independent data collected: 2974 , nb of independent data used for refinement: 2956 in the final least-squares refinement cycles on $\mathrm{F}^{2}$, the model converged at $R_{1}=0.0260, w R_{2}=0.0678[\mathrm{I}>2 \sigma(\mathrm{I})], R_{1}=0.0262, w R_{2}=0.0680$ (all data), and GOF $=1.076, \mathrm{H}$-atom parameters constrained.

CCDC 2006518 (for $\mathbf{4 m}$ ) contains the supplementary crystallographic data for this paper. These data can be obtained free of charge from The Cambridge Crystallographic Data Centre.

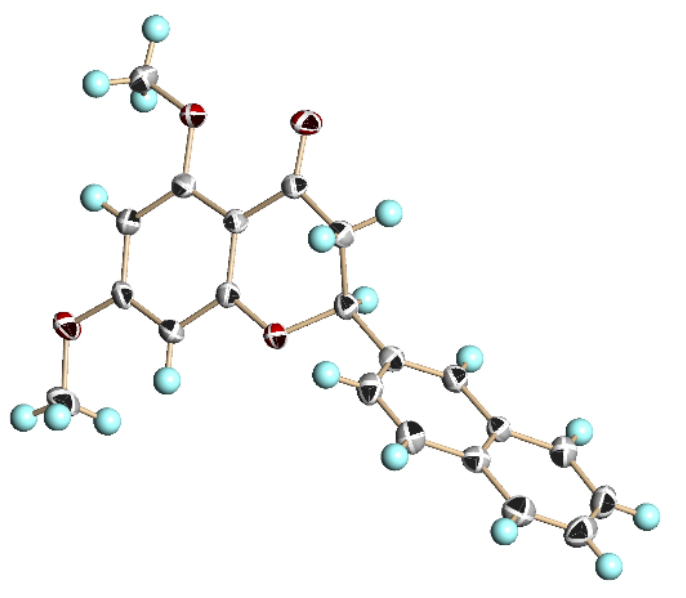

Figure S25. Perspective view of (S)-4m.

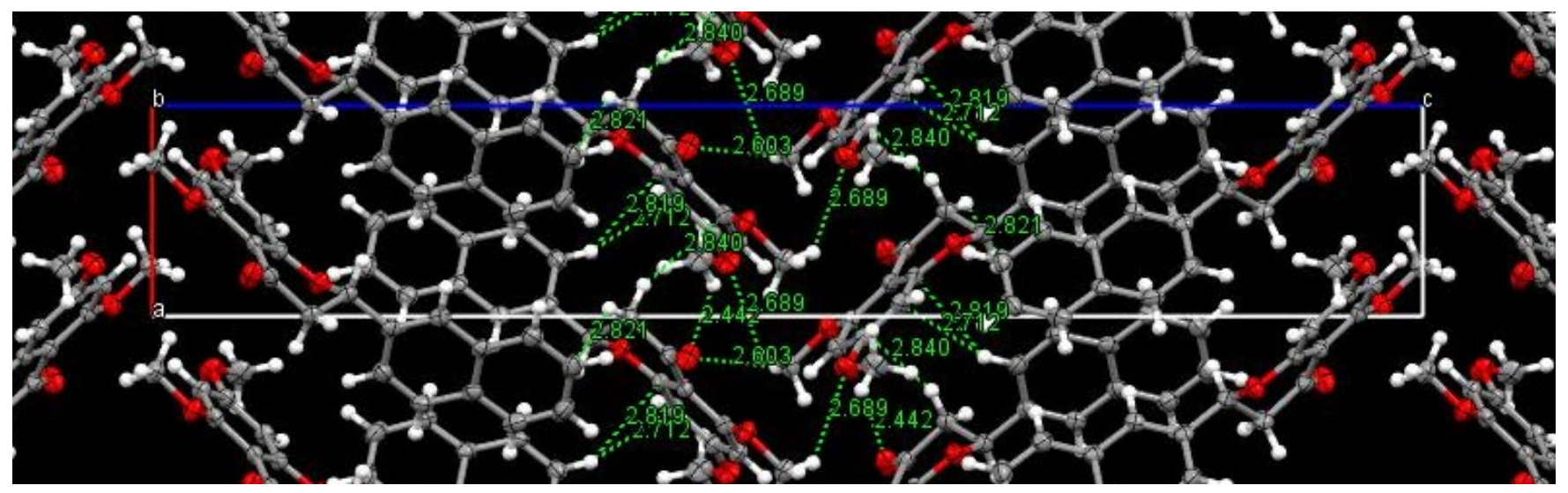

Figure S26. Packing diagram of (S)-4m along the $b$-axis. 


\section{Attrition-enhanced deracemization racemic flavanones 4}

Attrition-enhanced deracemization of racemic flavanone 4a. In a sealed glass tube ( $\varnothing 20 \mathrm{~mm}), 4 a(190.11 \mathrm{mg}, 0.67$ $\mathrm{mmol})$, propanol $(0.5 \mathrm{~mL})$, DBU $(0.05 \mathrm{~mL}, 0.33 \mathrm{mmol})$, and 20 glass beads $(\varnothing 2 \mathrm{~mm})$ were added and suspended with stirring at $600 \mathrm{rpm}$ using cross shape stirring bar $(15 \mathrm{~mm})$ at $60^{\circ} \mathrm{C}$. Crystals obtained by filtration were subjected to HPLC (CHIRALPAK IB, Hex: EtOH = $90: 10$, flow $1.0 \mathrm{~mL} / \mathrm{min}, \mathrm{RT}=10.1$ and $11.5 \mathrm{~min}$ ) to determine the enantiomeric purity.

Attrition-enhanced deracemization racemic flavanone 4c. In a sealed glass tube $(\varnothing 20 \mathrm{~mm}), 4 \mathrm{c}(199.5 \mathrm{mg}, 0.67$ $\mathrm{mmol})$, propanol $(0.5 \mathrm{~mL}), \mathrm{DBU}(0.05 \mathrm{~mL}, 0.33 \mathrm{mmol})$, and 20 glass beads $(\varnothing 2 \mathrm{~mm})$ were added and suspended with stirring at $600 \mathrm{rpm}$ using cross shape stirring bar $(15 \mathrm{~mm})$ at $80^{\circ} \mathrm{C}$. Crystals obtained by filtration were subjected to HPLC (CHIRALPAK IB, Hex : EtOH = $90: 10$, flow $1.0 \mathrm{~mL} / \mathrm{min}, \mathrm{RT}=9.2$ and $10.3 \mathrm{~min}$ ) to determine the enantiomeric purity.

Attrition-enhanced deracemization racemic flavanone $4 \mathrm{~h}$. In a sealed glass tube $(\varnothing 20 \mathrm{~mm}), 4 \mathrm{c}(200.0 \mathrm{mg}, 0.66$ $\mathrm{mmol})$, propanol $(0.5 \mathrm{~mL})$, DBU $(0.02 \mathrm{~mL}, 0.13 \mathrm{mmol})$, and 20 glass beads $(\varnothing 2 \mathrm{~mm})$ were added and suspended with stirring at $600 \mathrm{rpm}$ using cross shape stirring bar $(15 \mathrm{~mm})$ at $80^{\circ} \mathrm{C}$. Crystals obtained by filtration were subjected to HPLC (CHIRALPAK IB, Hex : EtOH = $90: 10$, flow $1.0 \mathrm{~mL} / \mathrm{min}$, RT = 11.5 and $13.2 \mathrm{~min}$ ) to determine the enantiomeric purity.

Attrition-enhanced deracemization racemic flavanone $4 I$. In a sealed glass tube $(\varnothing 20 \mathrm{~mm}), 4 \mathrm{I}(200.0 \mathrm{mg}, 0.60 \mathrm{mmol})$, propanol $(0.8 \mathrm{~mL})$, DBU $(0.036 \mathrm{~mL}, 0.24 \mathrm{mmol})$, and 20 glass beads $(\varnothing 2 \mathrm{~mm})$ were added and suspended with stirring at $600 \mathrm{rpm}$ using cross shape stirring bar $(15 \mathrm{~mm})$ at $80^{\circ} \mathrm{C}$. Crystals obtained by filtration were subjected to HPLC (CHIRALPAK IB, Hex : EtOH = $90: 10$, flow $1.0 \mathrm{~mL} / \mathrm{min}$, RT = 12.9 and $14.5 \mathrm{~min}$ ) to determine the enantiomeric purity.

Attrition-enhanced deracemization racemic flavanone $\mathbf{4 m}$. In a sealed glass tube $(\varnothing 20 \mathrm{~mm}), \mathbf{4 m}(223.4 \mathrm{mg}, 0.67$ $\mathrm{mmol})$, propanol $(1.2 \mathrm{~mL})$, DBU $(0.05 \mathrm{~mL}, 0.33 \mathrm{mmol}$ or $0.15 \mathrm{~mL}, 0.67 \mathrm{mmol})$, and 20 glass beads $(\varnothing 2 \mathrm{~mm})$ were added and suspended with stirring at $600 \mathrm{rpm}$ using cross shape stirring bar $(15 \mathrm{~mm})$ at $90^{\circ} \mathrm{C}$. Crystals obtained by filtration were subjected to HPLC (CHIRALPAK IB, Hex : EtOH = $90: 10$, flow $1.0 \mathrm{~mL} / \mathrm{min}, \mathrm{RT}=14.7$ and 17.7 $\min$ ) to determine the enantiomeric purity.

One-pot absolute asymmetric synthesis of $\mathbf{4 m}$ from prochiral aldol reaction product $3 \mathrm{~m}$ by reversible oxa-Michael addition followed by attrition-enhanced deracemization. In a sealed glass tube $(\varnothing 20 \mathrm{~mm}), 3 \mathrm{~m}(200 \mathrm{mg}, 0.60 \mathrm{mmol})$, propanol $(1.2 \mathrm{~mL}), \mathrm{DBU}(0.13 \mathrm{~mL}, 0.60 \mathrm{mmol})$, and 20 glass beads $(\varnothing 2 \mathrm{~mm})$ were added and suspended with stirring at $600 \mathrm{rpm}$ using cross shape stirring bar $(15 \mathrm{~mm})$ at $90^{\circ} \mathrm{C}$. Crystals obtained by filtration were subjected to HPLC (CHIRALPAK IB, Hex : EtOH = $90: 10$, flow $1.0 \mathrm{~mL} / \mathrm{min}, \mathrm{RT}=14.7$ and $17.7 \mathrm{~min}$ ) to determine the enantiomeric purity.

One-pot absolute asymmetric synthesis of $\mathbf{4 m}$ from $\mathbf{1}$ and $\mathbf{2 m}$ by aldol reaction, reversible oxa-Michael addition, and attrition-enhanced deracemization. To a sealed glass tube, 1 (210 mg, $1.07 \mathrm{mmol}), 2 \mathrm{~m}$ (167.3 mg, $1.07 \mathrm{~mol})$, methanol $(3 \mathrm{~mL}), \mathrm{DBU}(0.23 \mathrm{~mL}, 1.50 \mathrm{mmol})$, and glass beads were added and stirred at room temperature for 24 h. After the solvent was removed in vacuo, 1-propanol $(1.0 \mathrm{~mL})$ was added and the solids were suspended with with stirring at $600 \mathrm{rpm}$ using cross shape stirring bar $(15 \mathrm{~mm})$ at $90{ }^{\circ} \mathrm{C}$ for several days. The ee value for the solid began to increase after 6 days and gradually reached $89 \%$ after 29 days. 


\section{References}

[S1] H, M. Sim, K, Y. Loh, W, K. Yeo, C, Y. Lee, M, L. Go, Aurones as modulators of ABCG2 and ABCB1: synthesis and structure-activity relationships, Chem. Med. Chem., 2011, 6, 713-724.

[S2] C. Yengai, S. Wanich, Cytotoxicity against KB and NCl-H187 cell lines of modified flavonoids from Kaempferia parviflora, Bioorg. Med. Chem., 2010, 20, 2821-2823.

[S3] H. Sekizaki, Synthesis of 2-Benzylidene-3(2H)-benzofuran-3-ones (Aurones) by Oxidation of 2'-Hydroxychalcones with Mercury(II) Acetate, Bull. Chem. Soc. Jpn., 1988, 61, 1407-1409. 
Figure 27. HPLC analysis of racemic $4 a$.

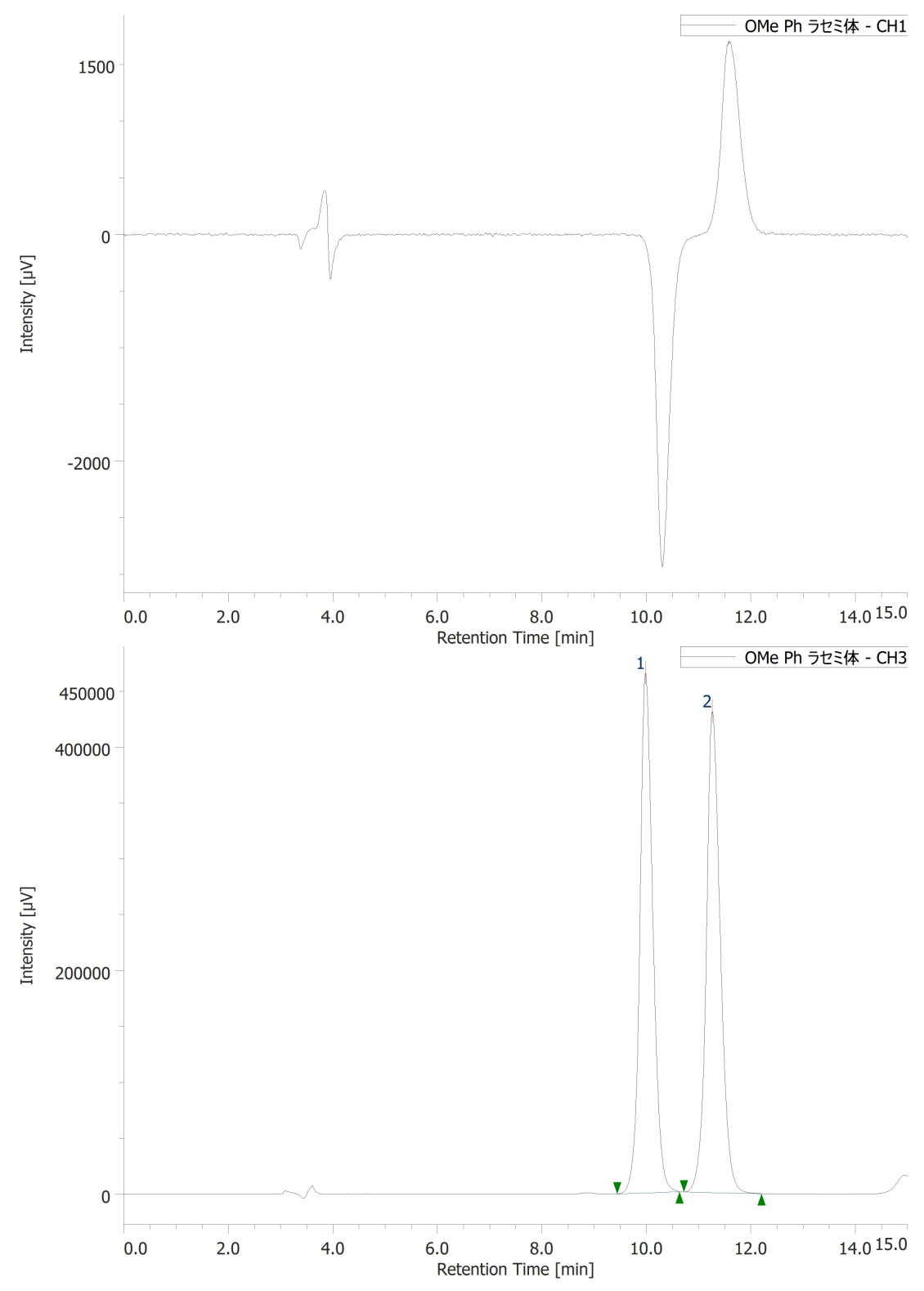

Column: CHIRALPAK-IB

Flow rate: $1.0 \mathrm{~mL} / \mathrm{min}$

Eluent: Hexane : EtOH = $90: 10$ 
Figure 28. HPLC analysis of $91 \%$ ee $\mathbf{4 a .}$

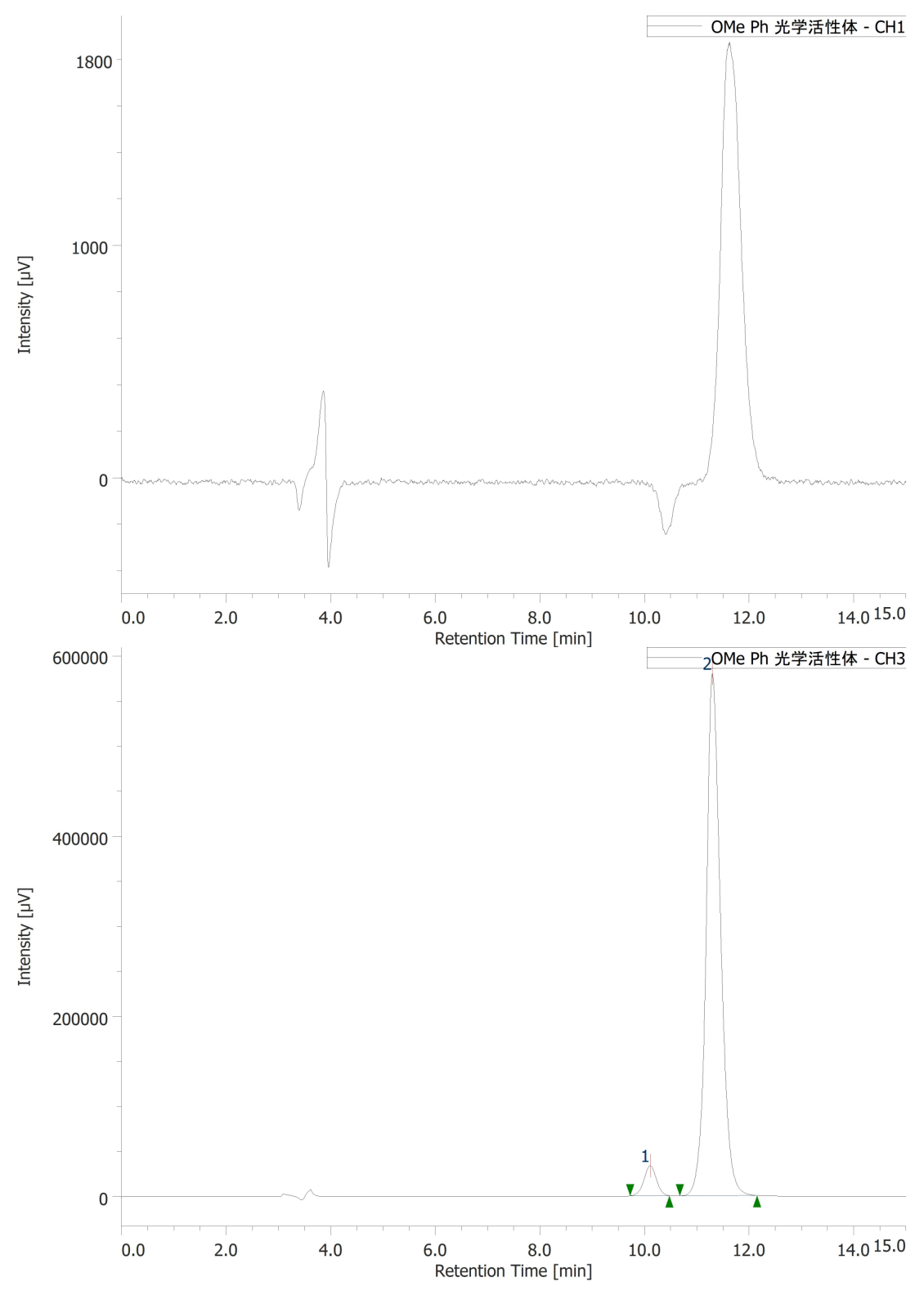

Column: CHIRALPAK-IB

Flow rate: $1.0 \mathrm{~mL} / \mathrm{min}$

Eluent: Hexane : EtOH = $90: 10$ 
Figure 29. HPLC analysis of racemic 4c.

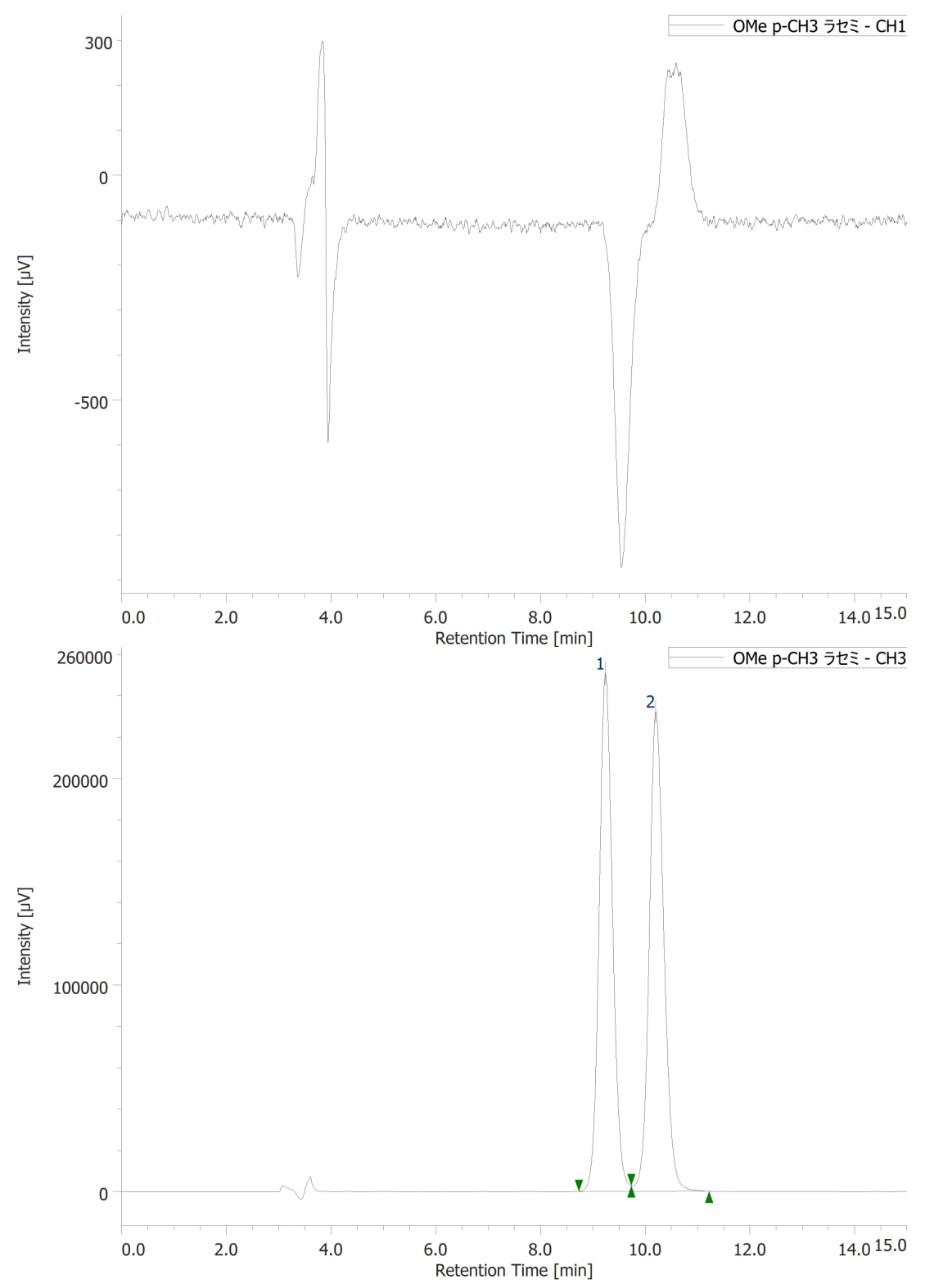

Column: CHIRALPAK-IB

Flow rate: $1.0 \mathrm{~mL} / \mathrm{min}$

Eluent: Hexane : EtOH = $90: 10$ 
Figure 30. HPLC analysis of $94 \%$ ee of $4 c$.

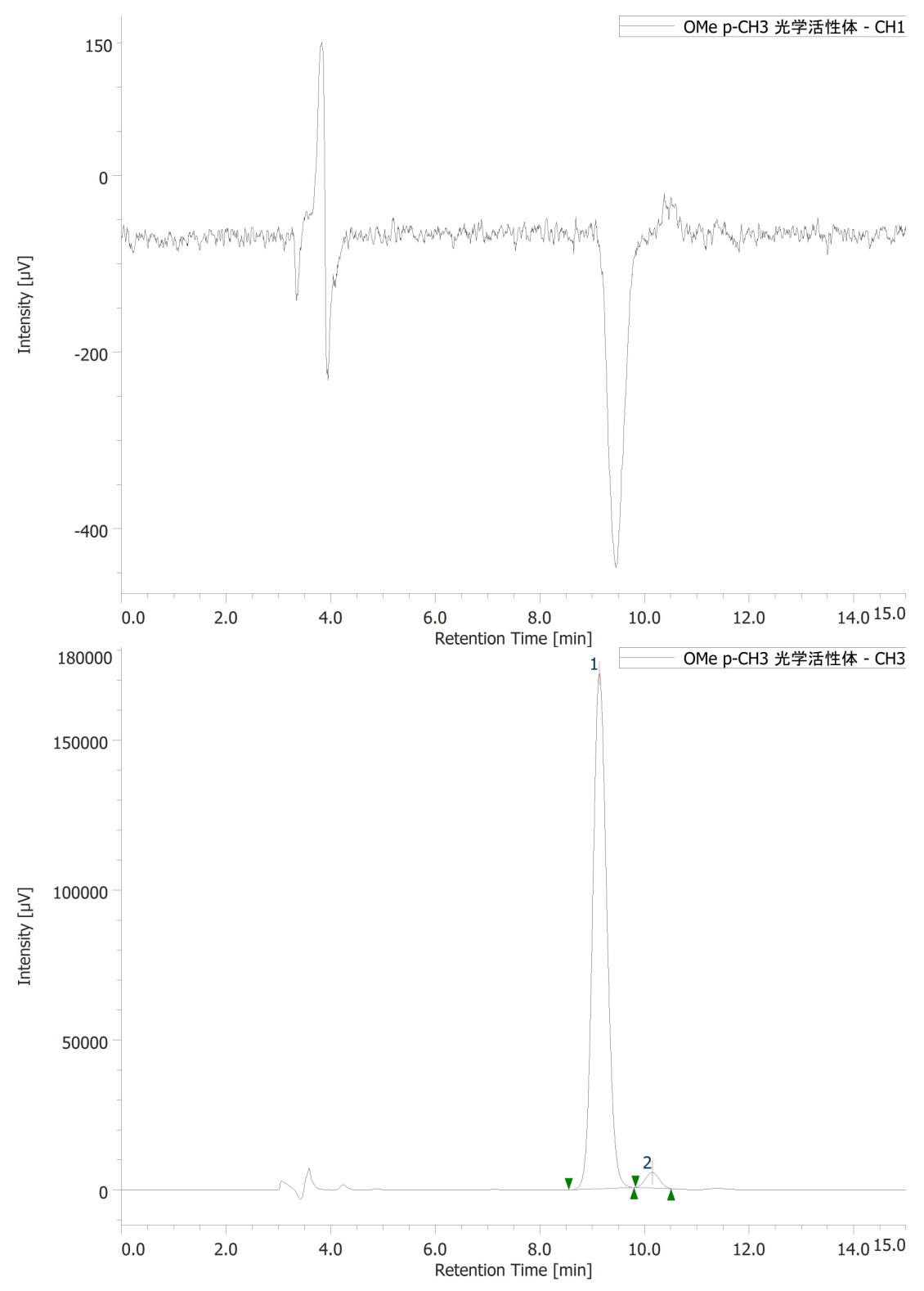

Column: CHIRALPAK-IB

Flow rate: $1.0 \mathrm{~mL} / \mathrm{min}$

Eluent: Hexane : EtOH $=90: 10$ 
Figure 31. HPLC analysis of racemic $4 \mathrm{~h}$.

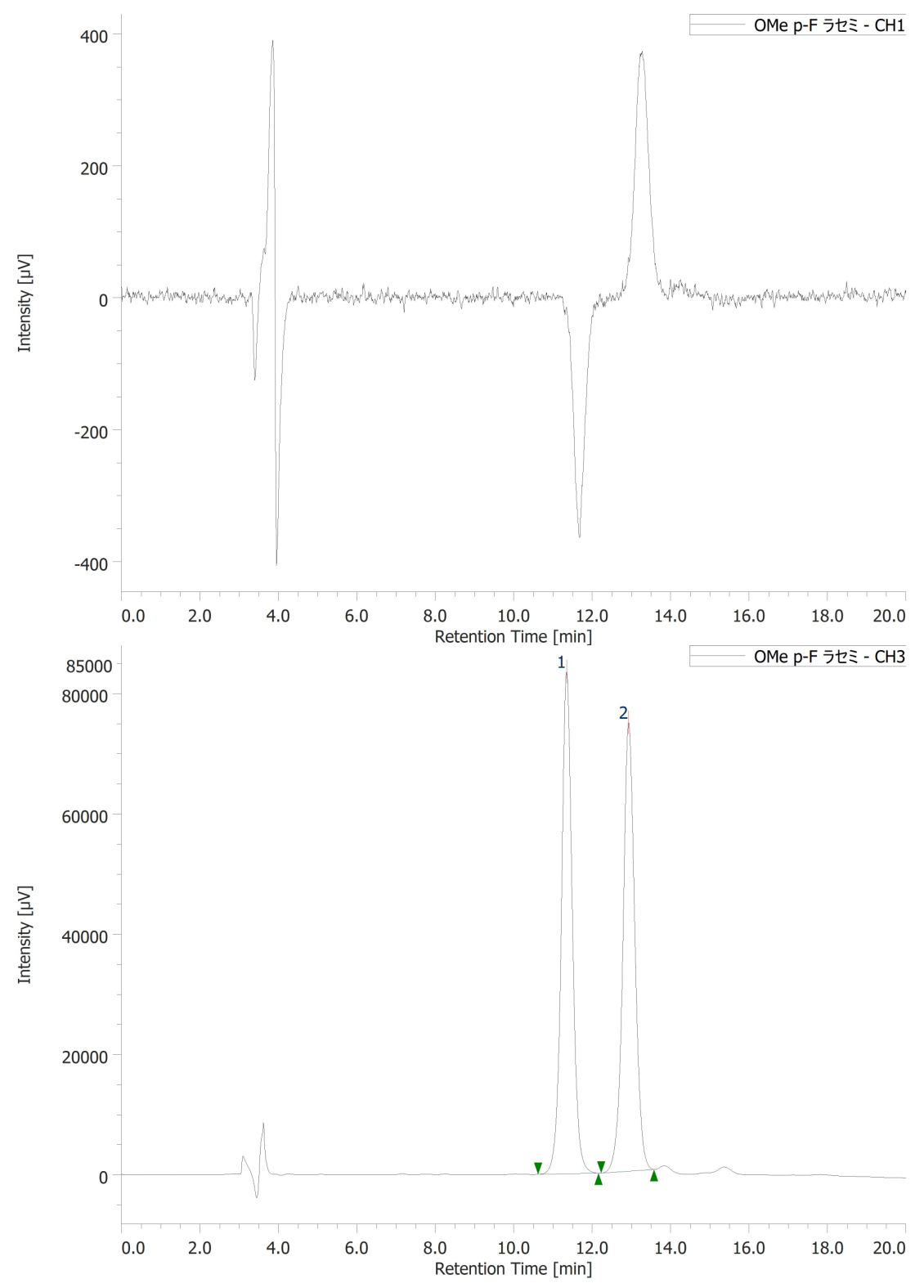

Column: CHIRALPAK-IB

Flow rate: $1.0 \mathrm{~mL} / \mathrm{min}$

Eluent: Hexane : $\mathrm{EtOH}=90: 10$ 
Figure 32. HPLC analysis of $78 \%$ ee of $\mathbf{4 h}$.

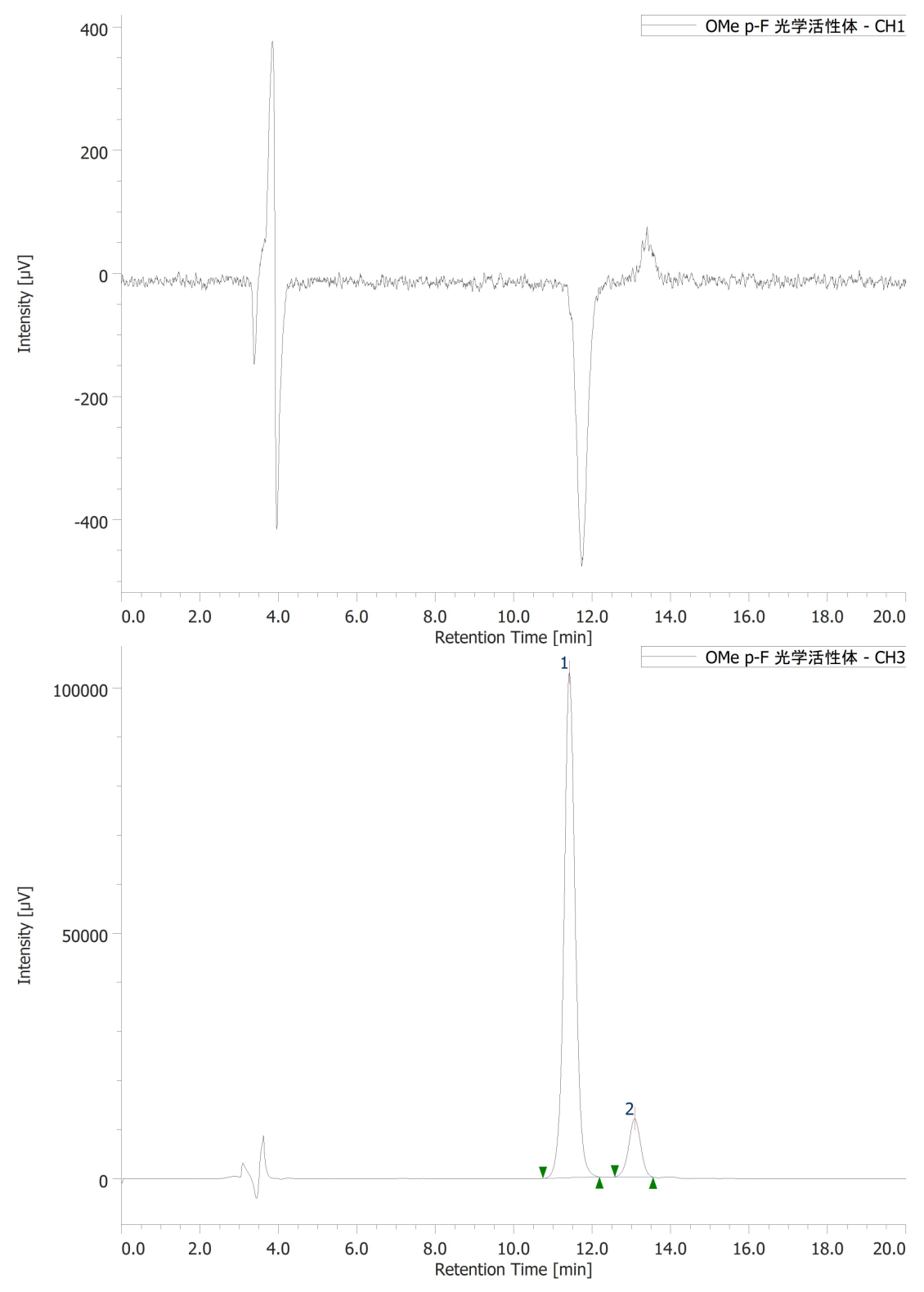

Column: CHIRALPAK-IB

Flow rate: $1.0 \mathrm{~mL} / \mathrm{min}$

Eluent: Hexane : EtOH $=90: 10$ 
Figure 33. HPLC analysis of racemic 4l.
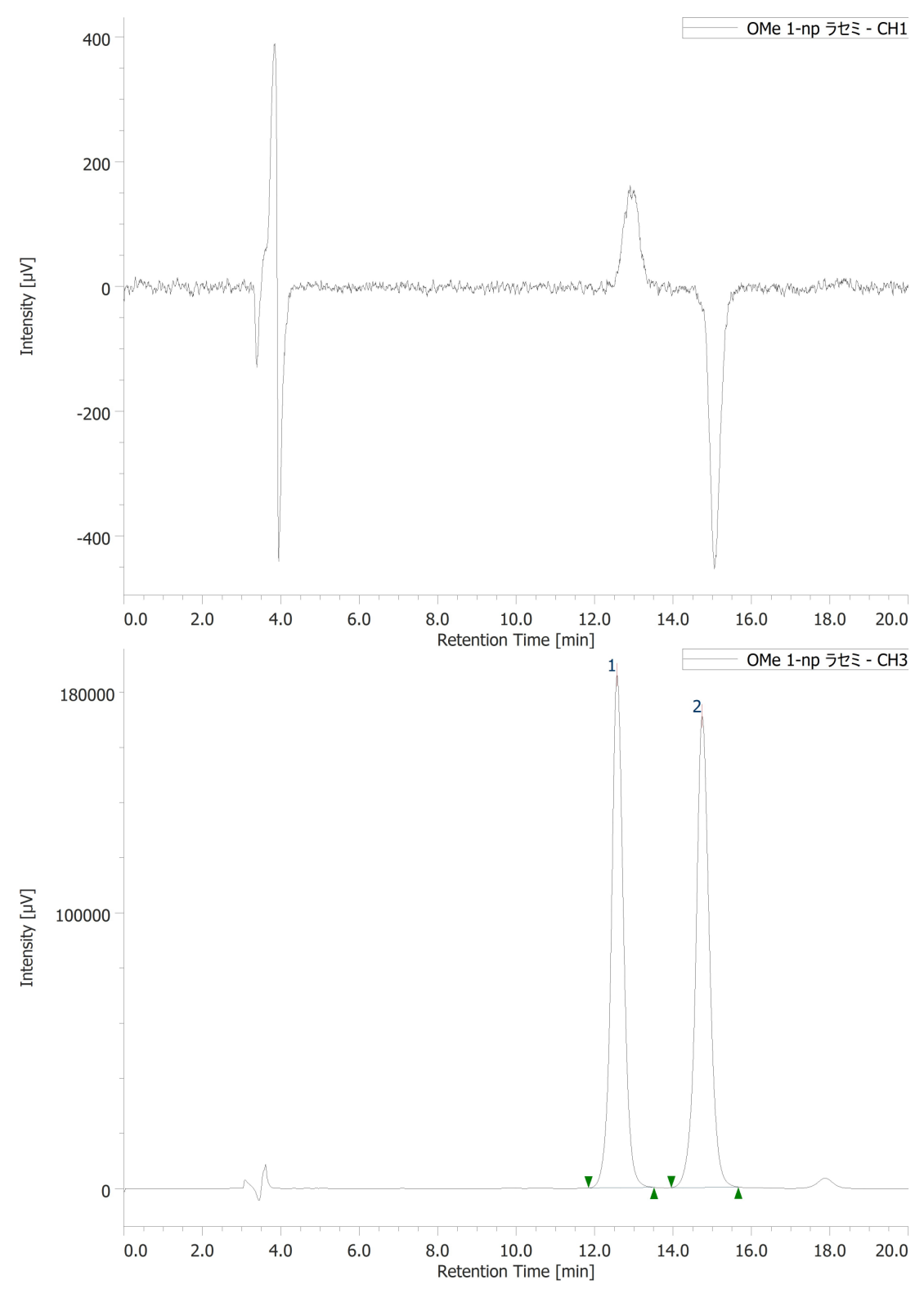

Column: CHIRALPAK-IB

Flow rate: $1.0 \mathrm{~mL} / \mathrm{min}$

Eluent: Hexane : EtOH = $90: 10$ 
Figure 34. HPLC analysis of $99 \%$ e of 41 .
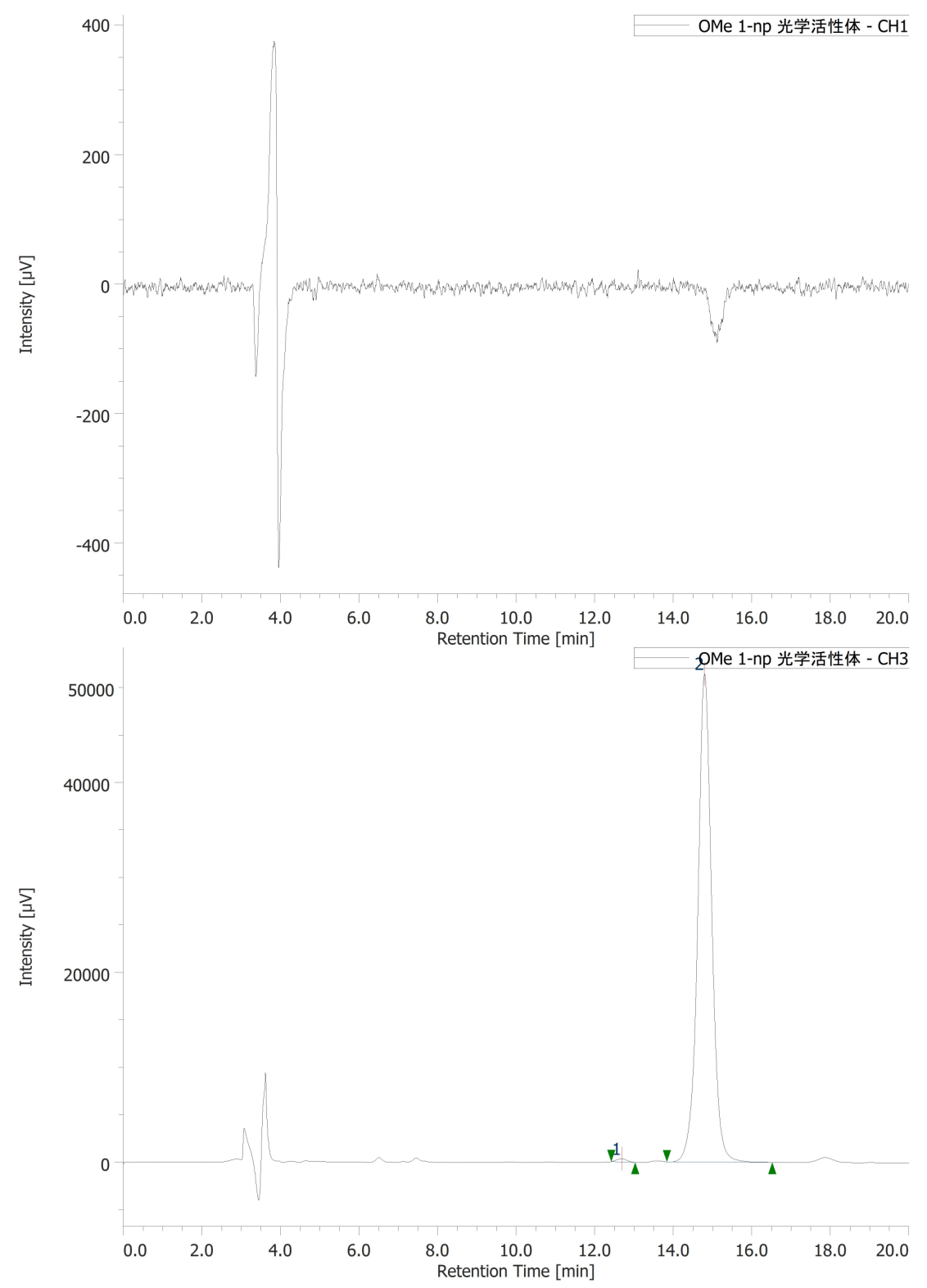

Column: CHIRALPAK-IB

Flow rate: $1.0 \mathrm{~mL} / \mathrm{min}$

Eluent: Hexane : EtOH $=90: 10$ 
Figure 35. HPLC analysis of racemic $\mathbf{4 m}$.

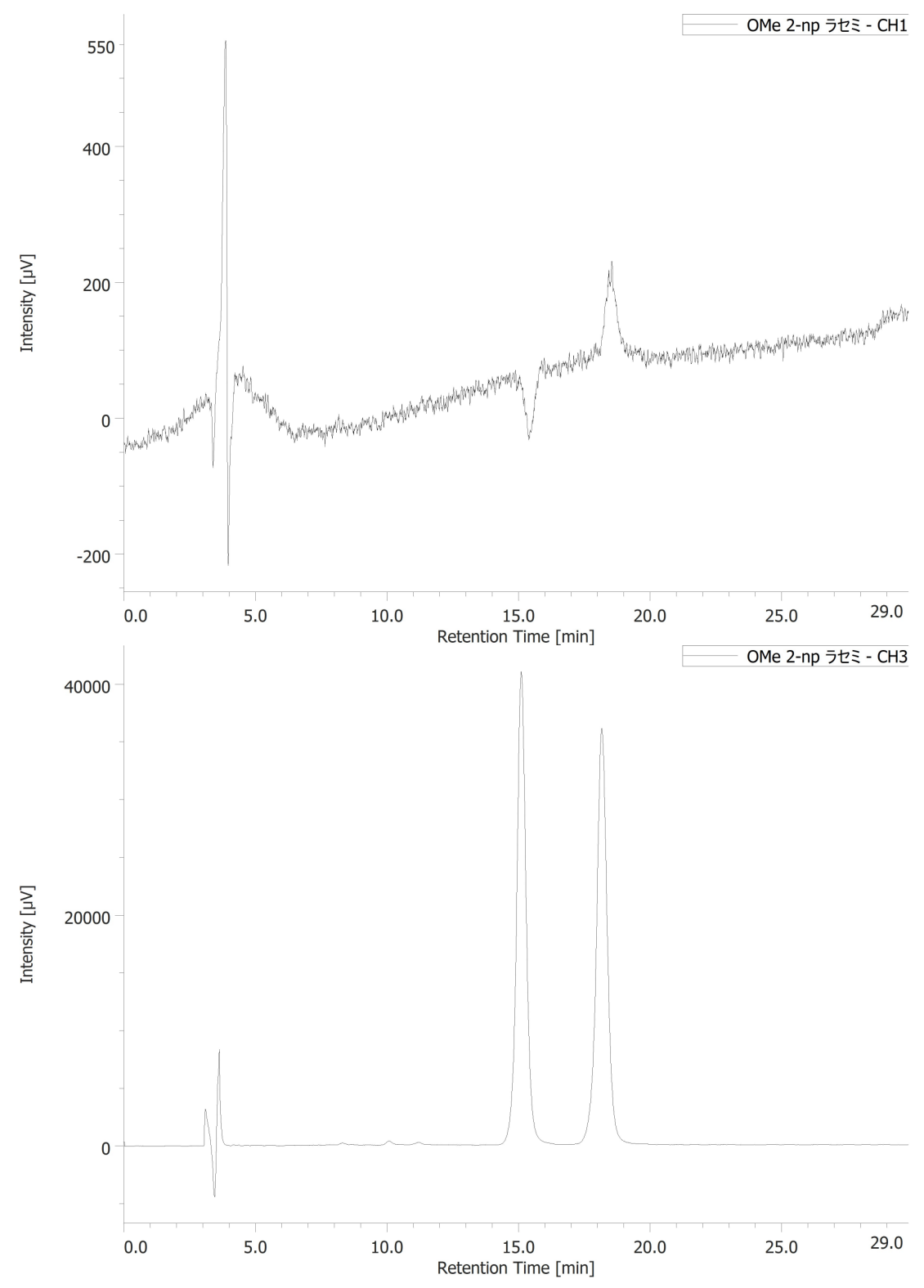

Column: CHIRALPAK-IB

Flow rate: $1.0 \mathrm{~mL} / \mathrm{min}$

Eluent: Hexane : $\mathrm{EtOH}=90: 10$ 
Figure 36. HPLC analysis of $98 \%$ ee of $4 \mathrm{~m}$.

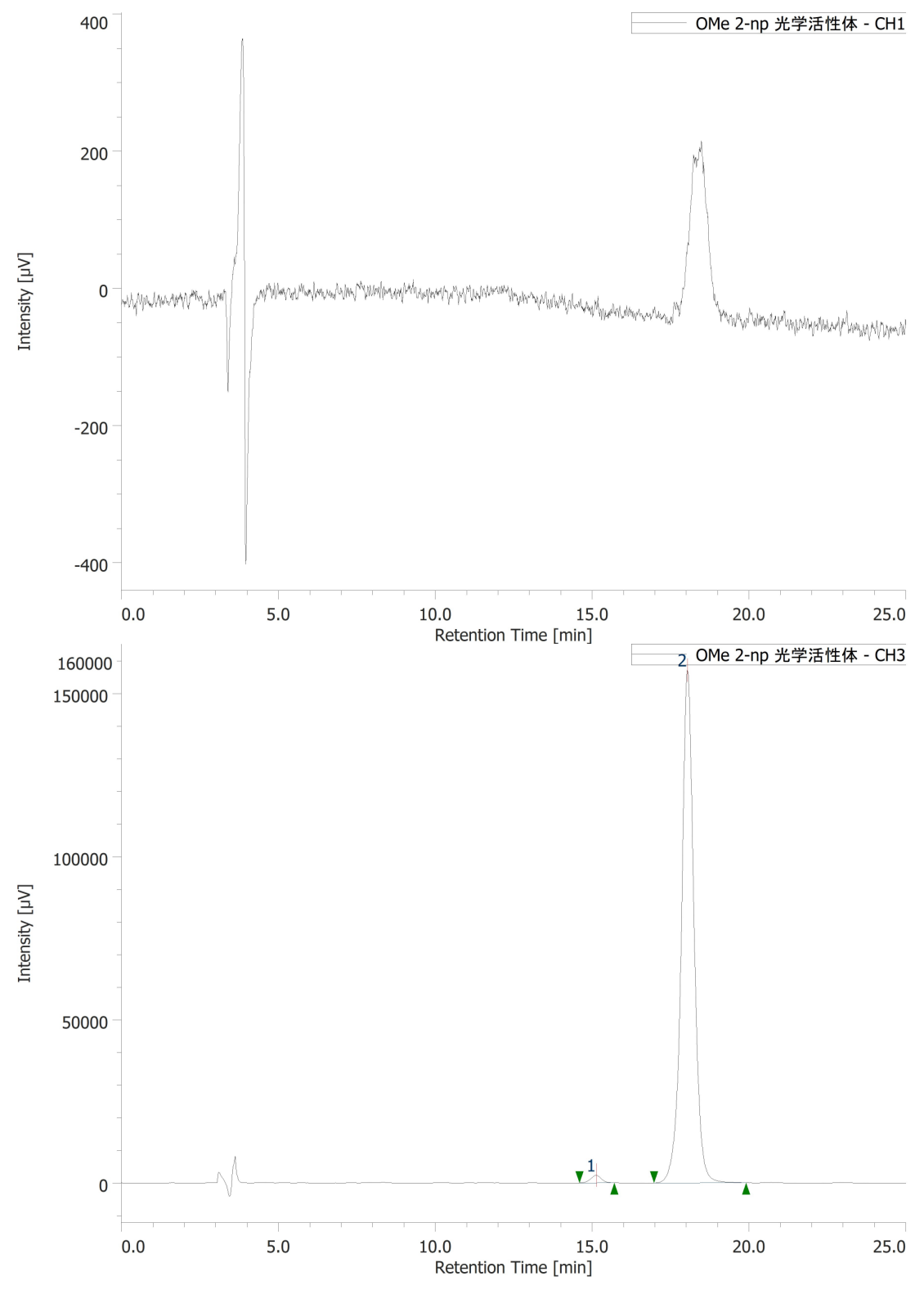

Column: CHIRALPAK-IB

Flow rate: $1.0 \mathrm{~mL} / \mathrm{min}$

Eluent: Hexane : $\mathrm{EtOH}=90: 10$ 
Figure $37 .{ }^{1} \mathrm{H}$ NMR of 3a

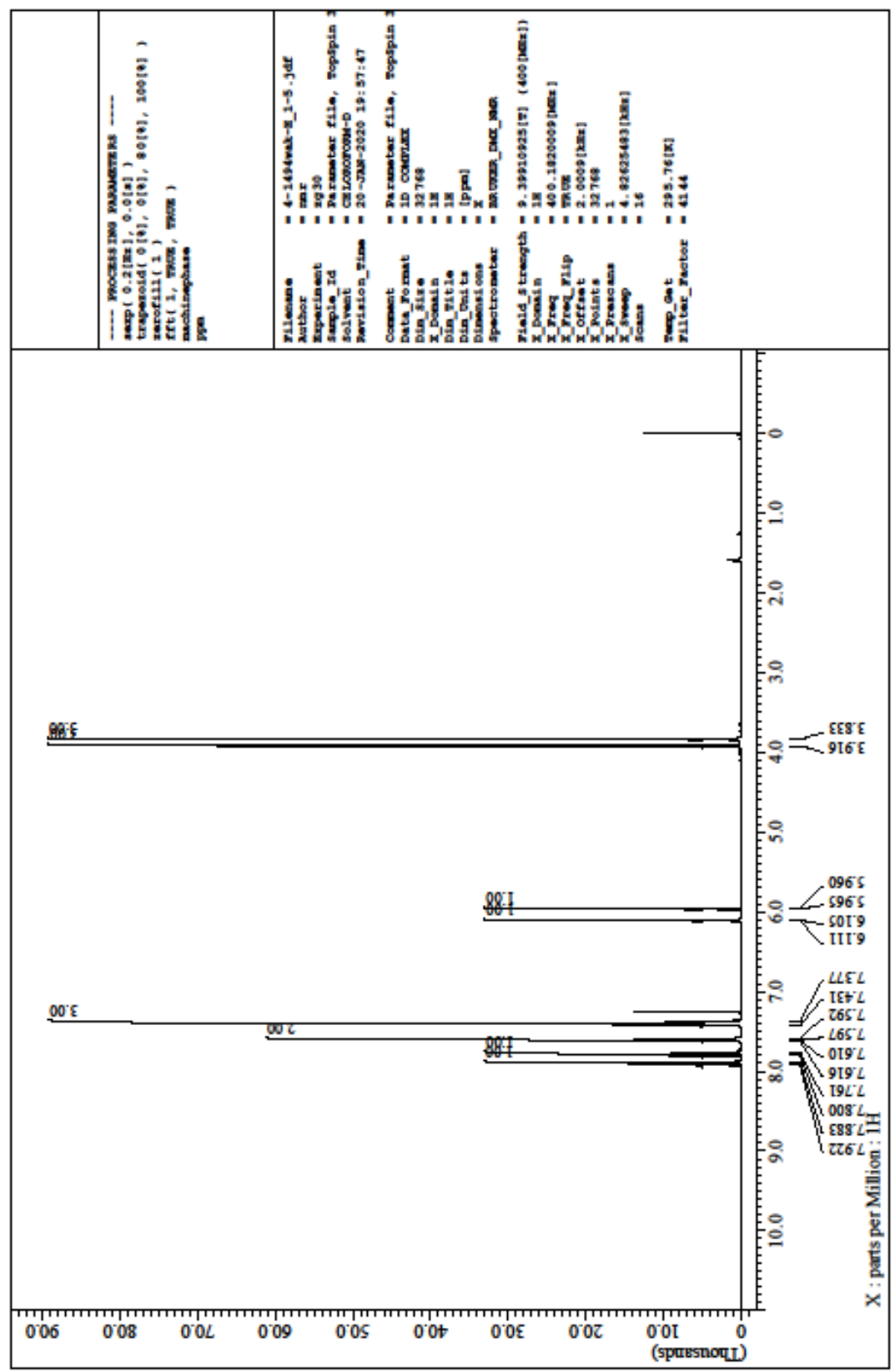


Figure $38 .{ }^{13} \mathrm{C}$ NMR of $3 a$

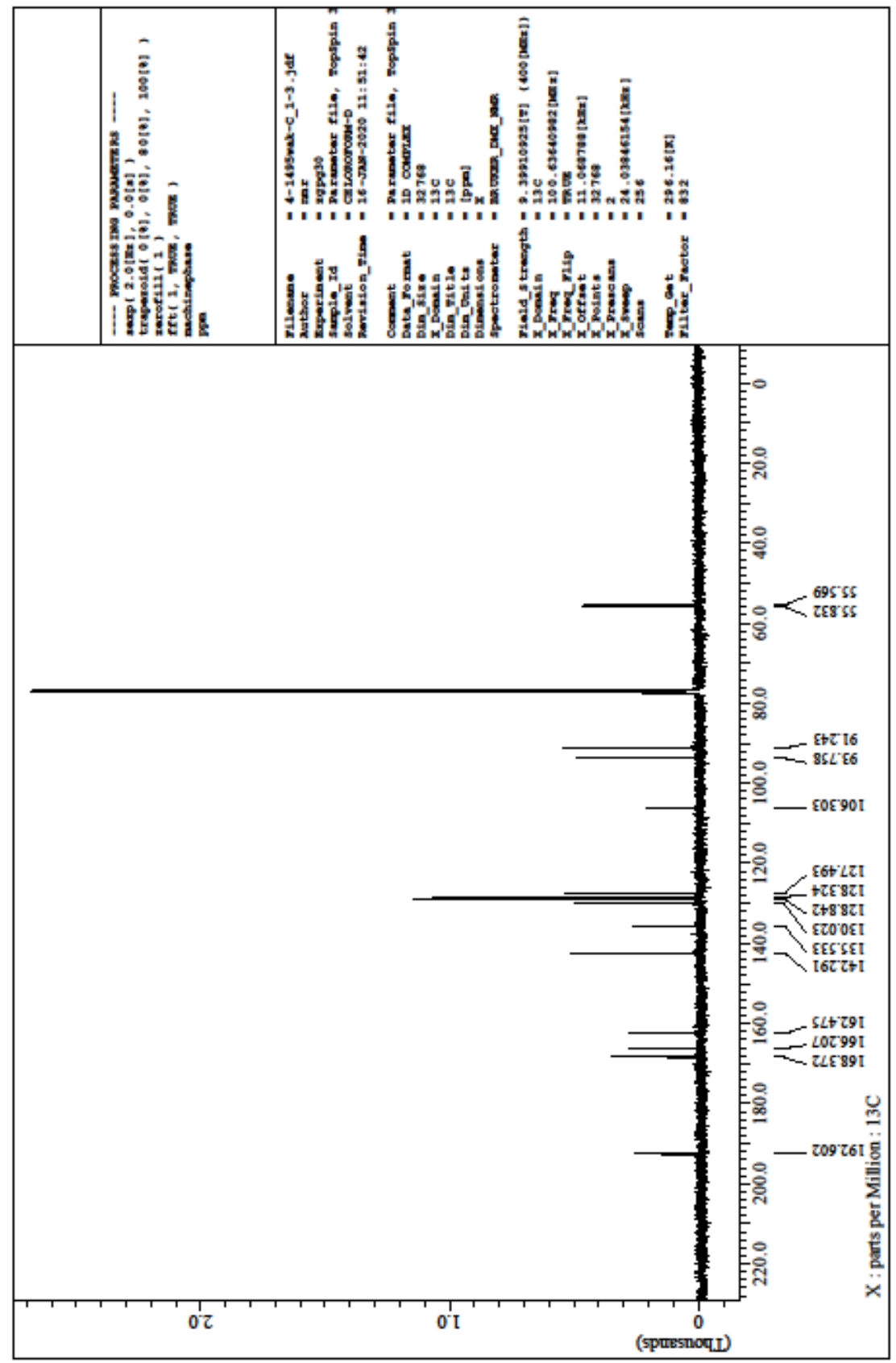


Figure 39. ${ }^{1} \mathrm{H}$ NMR of $3 \mathrm{c}$

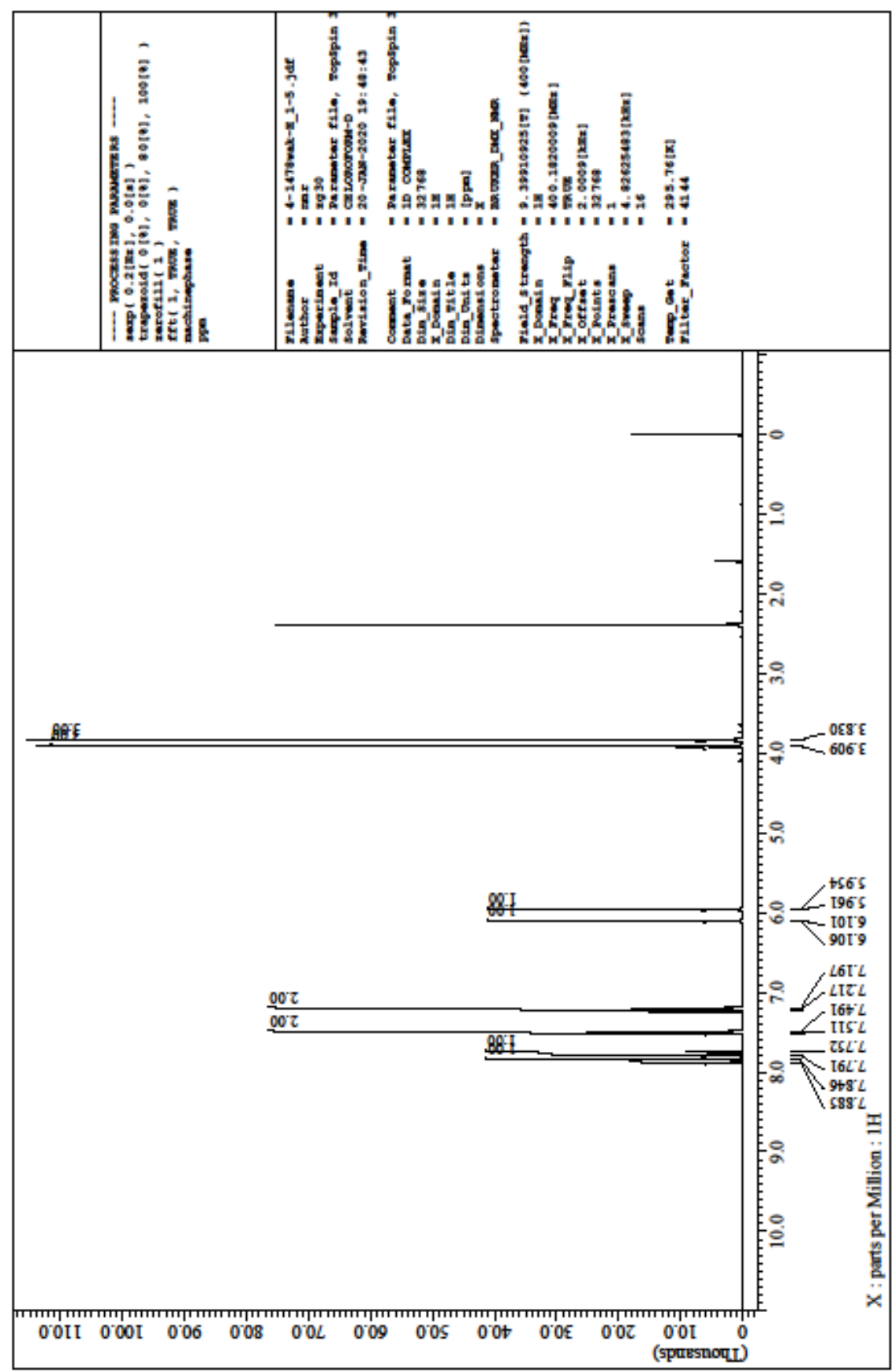


Figure $40 .{ }^{13} \mathrm{C}$ NMR of $3 \mathrm{C}$

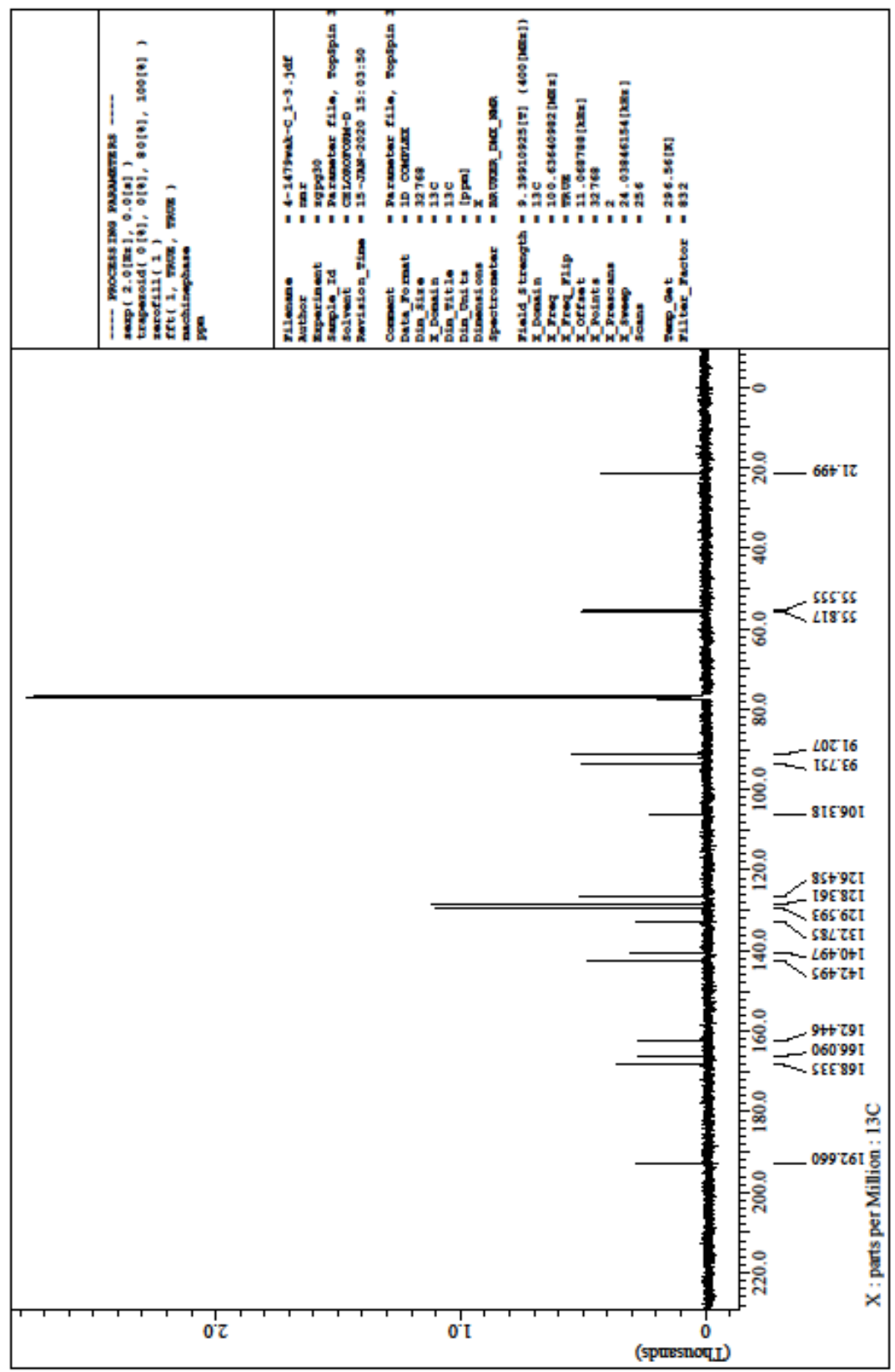


Figure 41. ${ }^{1} \mathrm{H}$ NMR of $3 \mathrm{~h}$

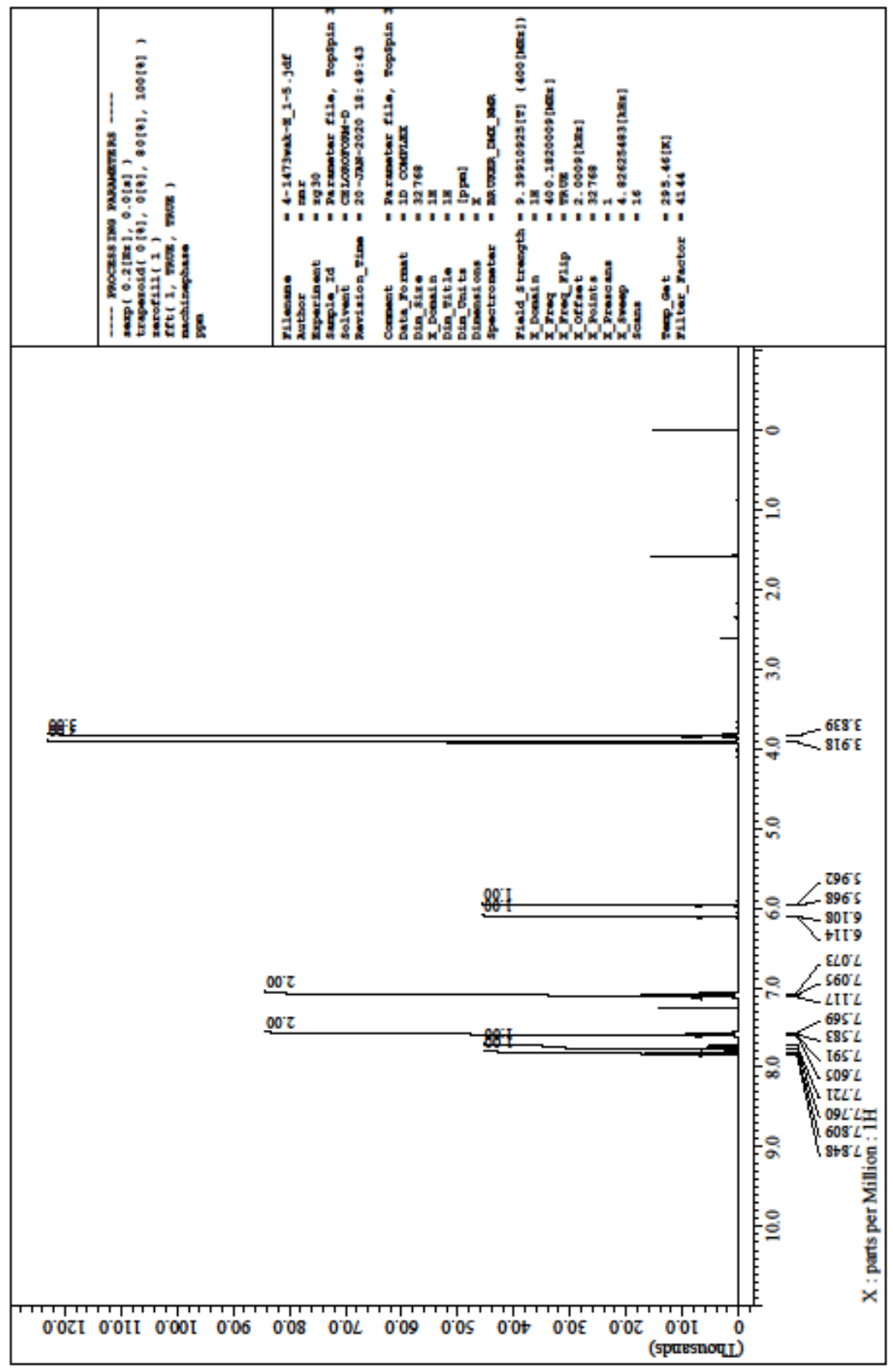


Figure 42. ${ }^{13} \mathrm{C}$ NMR of $3 \mathrm{~h}$

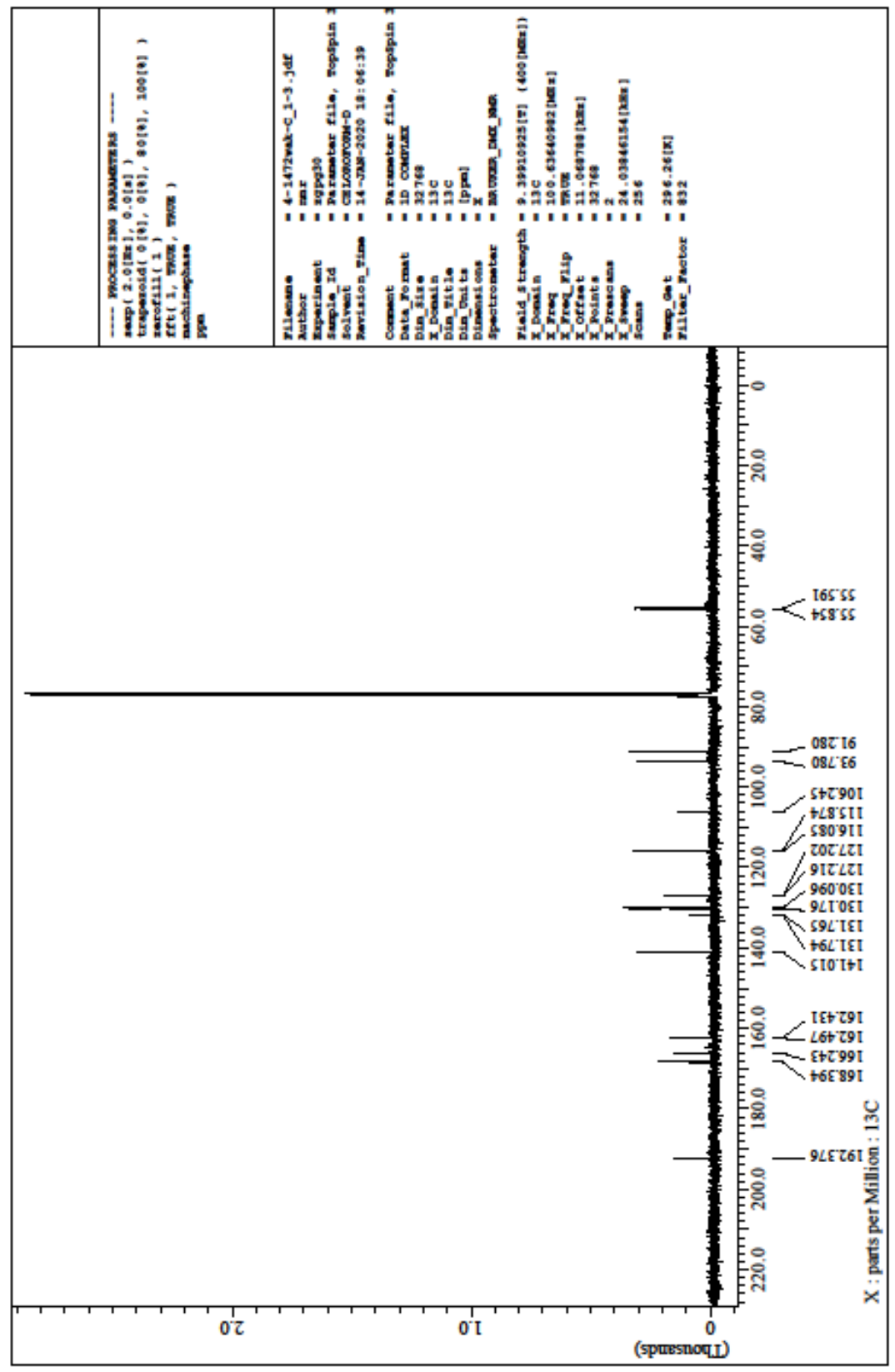


Figure 43. ${ }^{1} \mathrm{H}$ NMR of $\mathbf{3 j}$

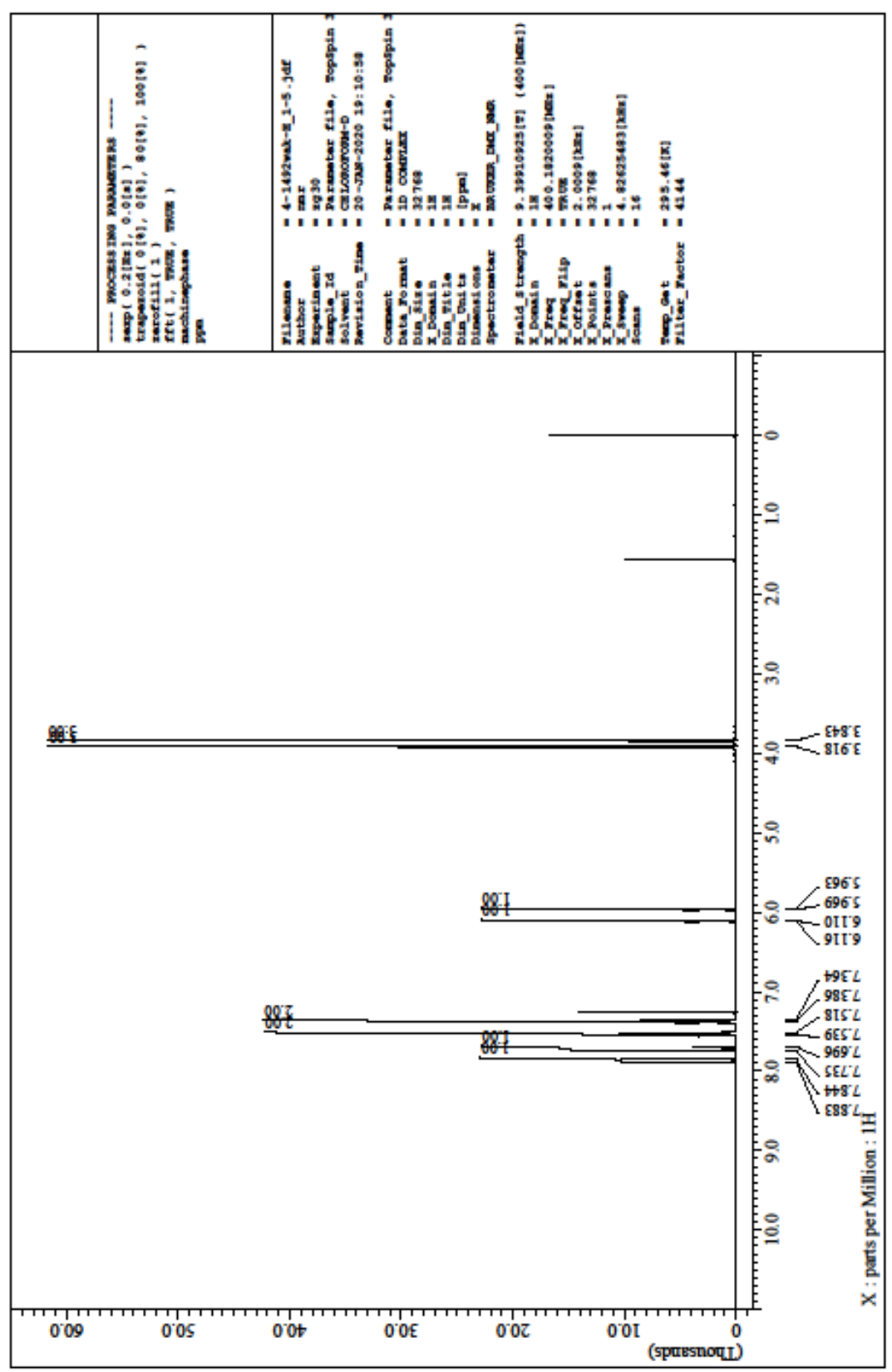


Figure $44 .{ }^{13} \mathrm{C}$ NMR of $\mathbf{3 j}$

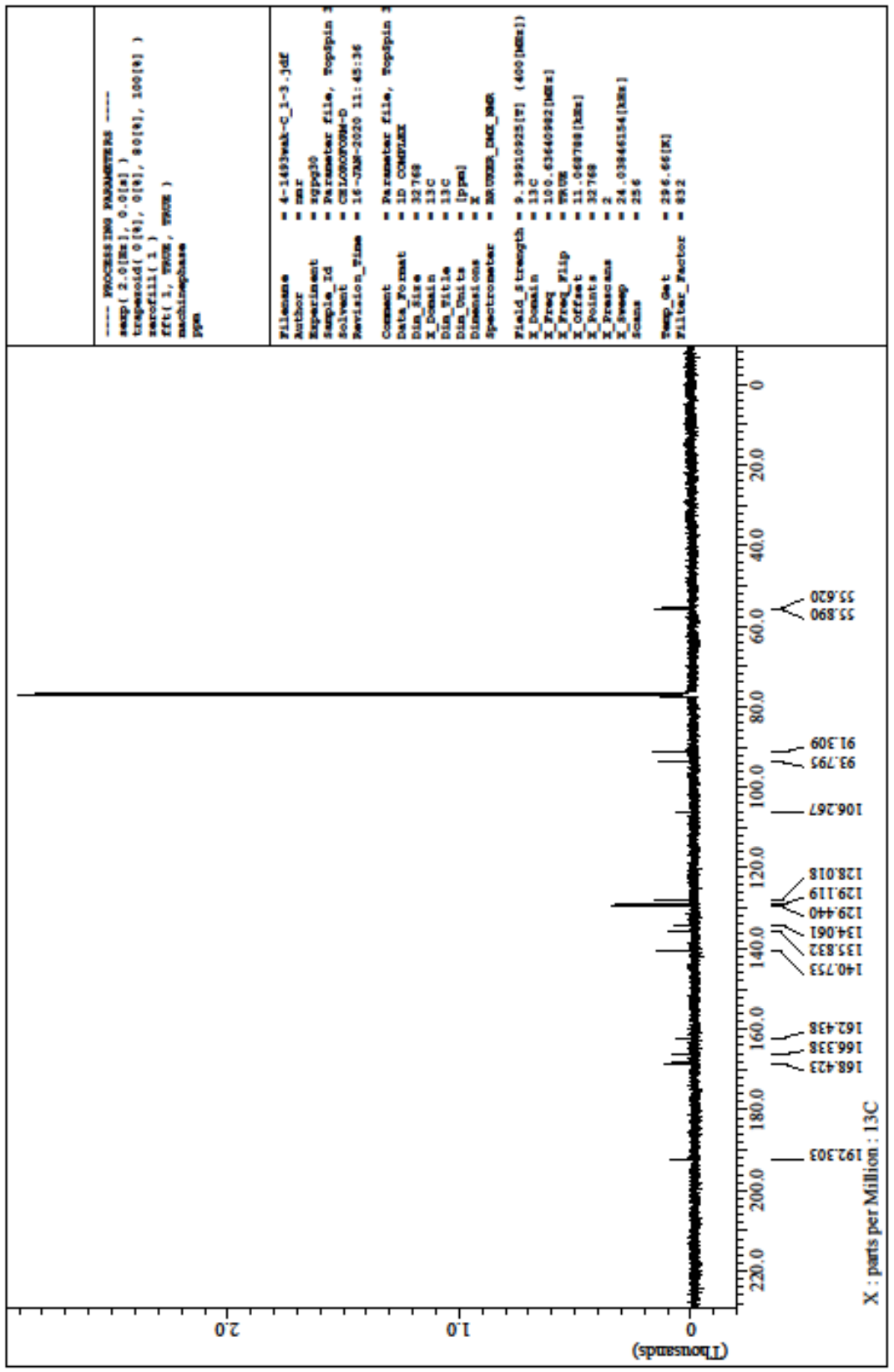


Figure 45. ${ }^{1} \mathrm{H}$ NMR of $3 k$

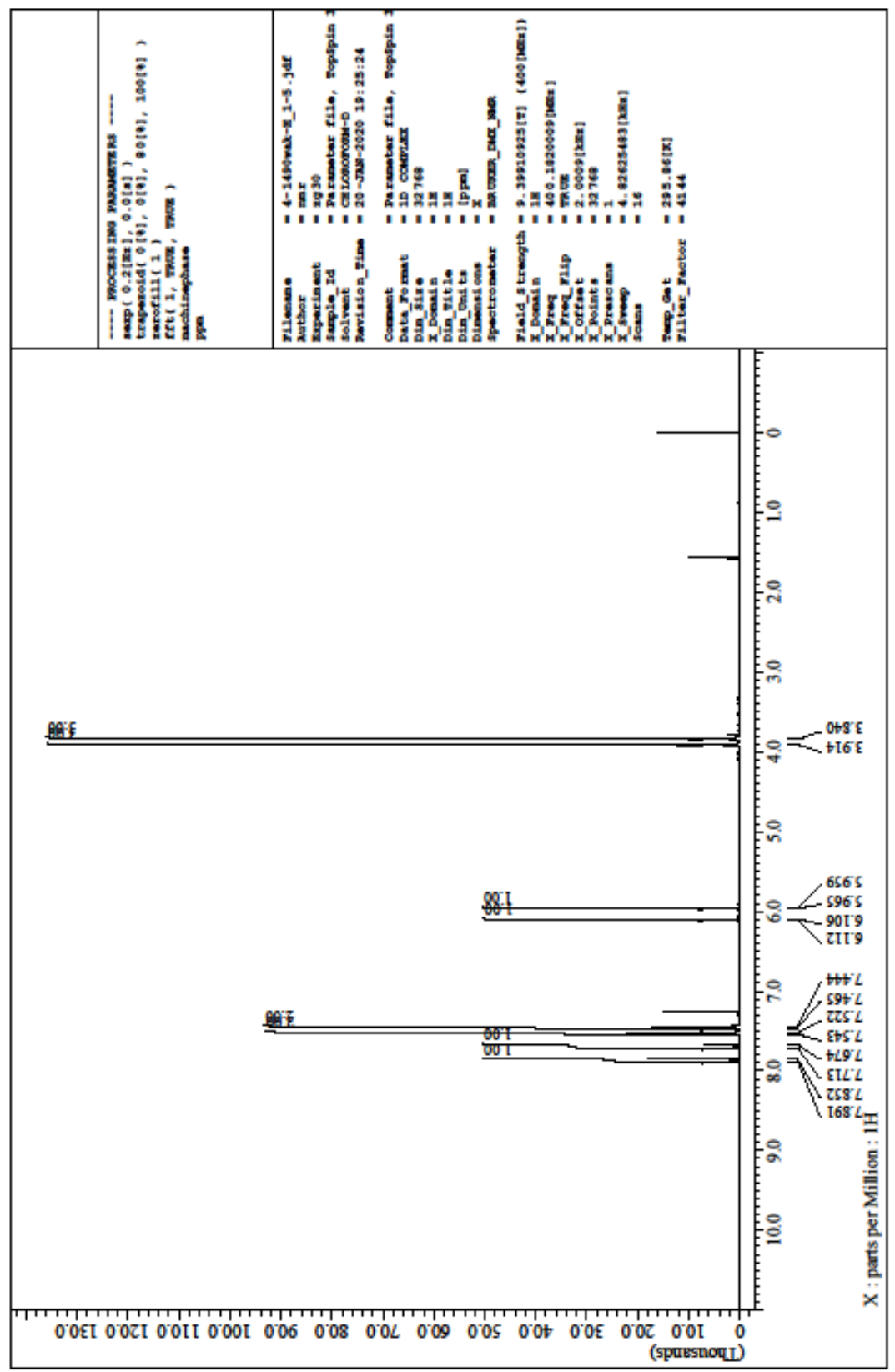


Figure 46. ${ }^{13} \mathrm{C}$ NMR of $3 k$

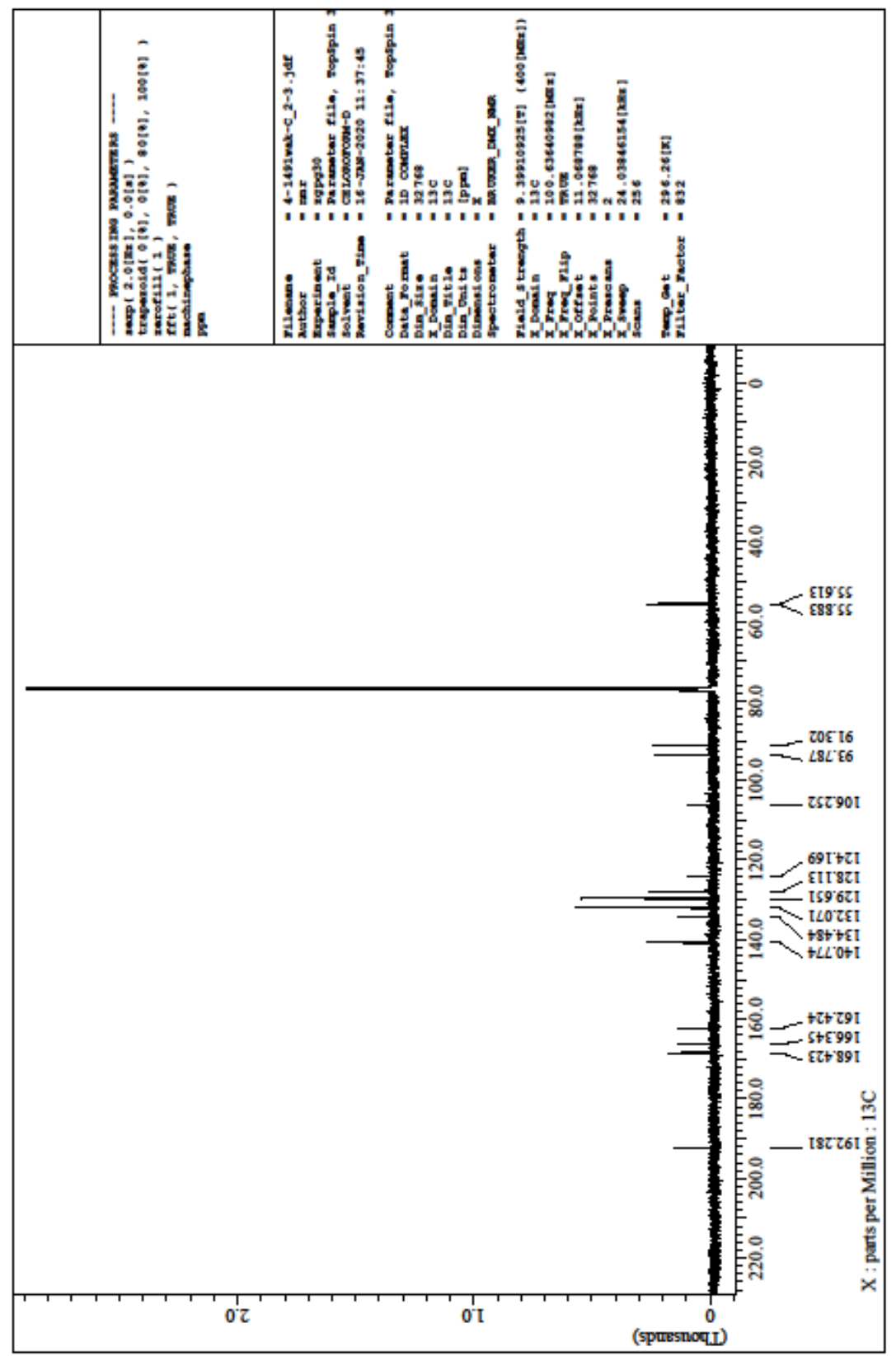


Figure 47. ${ }^{1} \mathrm{H}$ NMR of $3 \mathrm{I}$

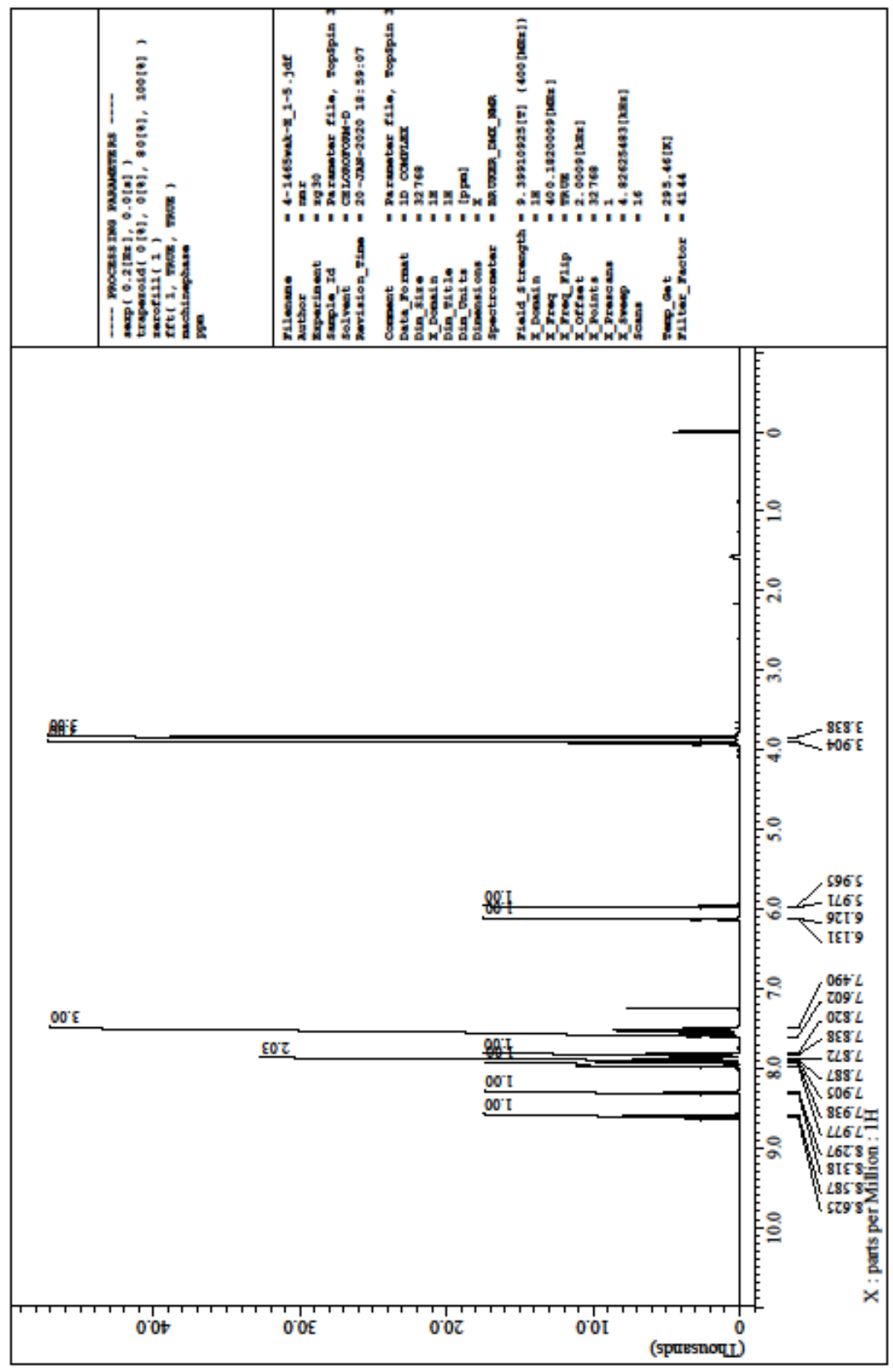


Figure 48. ${ }^{13} \mathrm{C}$ NMR of $3 \mathrm{I}$

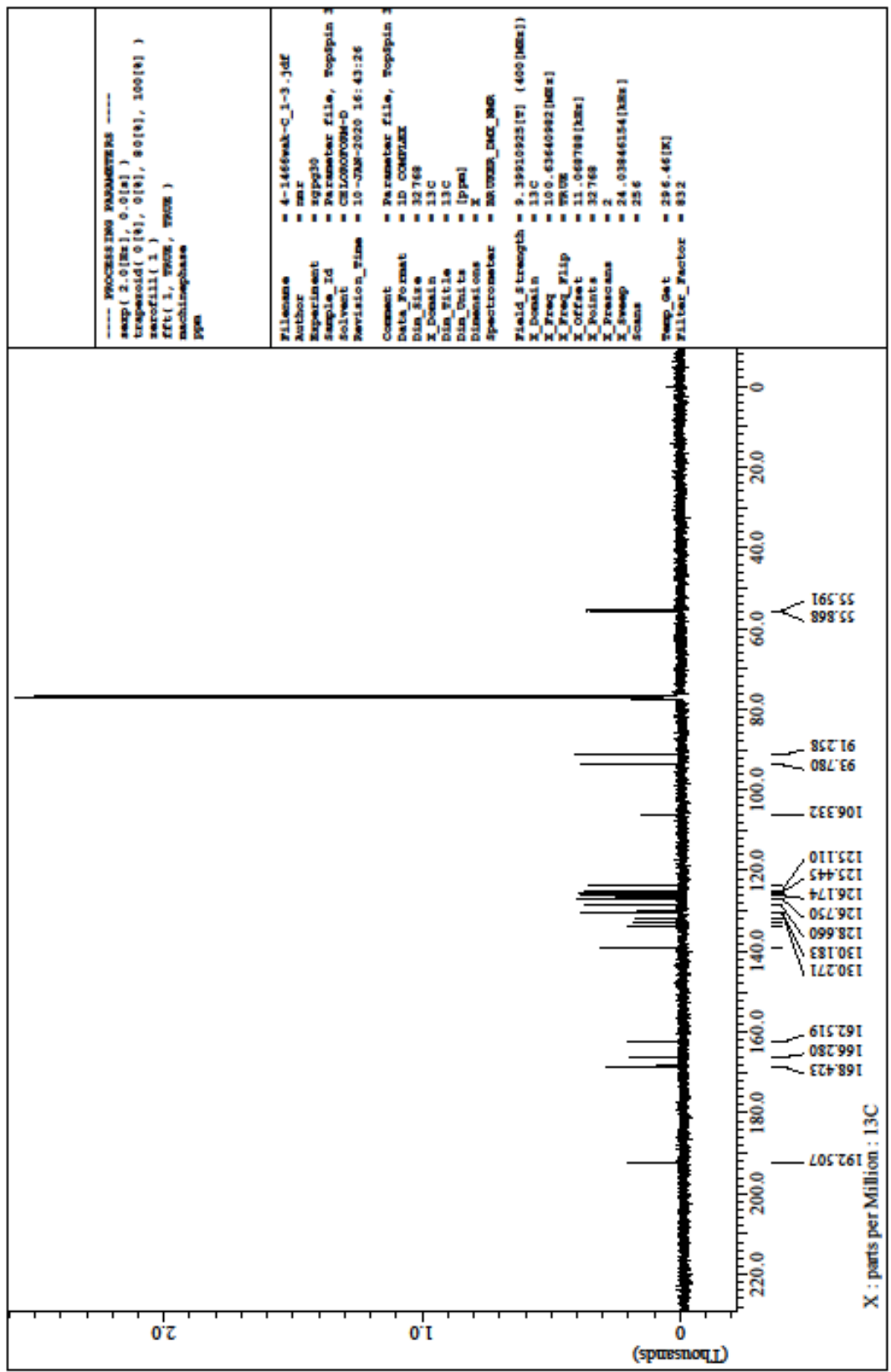


Figure 49. ${ }^{1} \mathrm{H}$ NMR of $3 \mathrm{~m}$

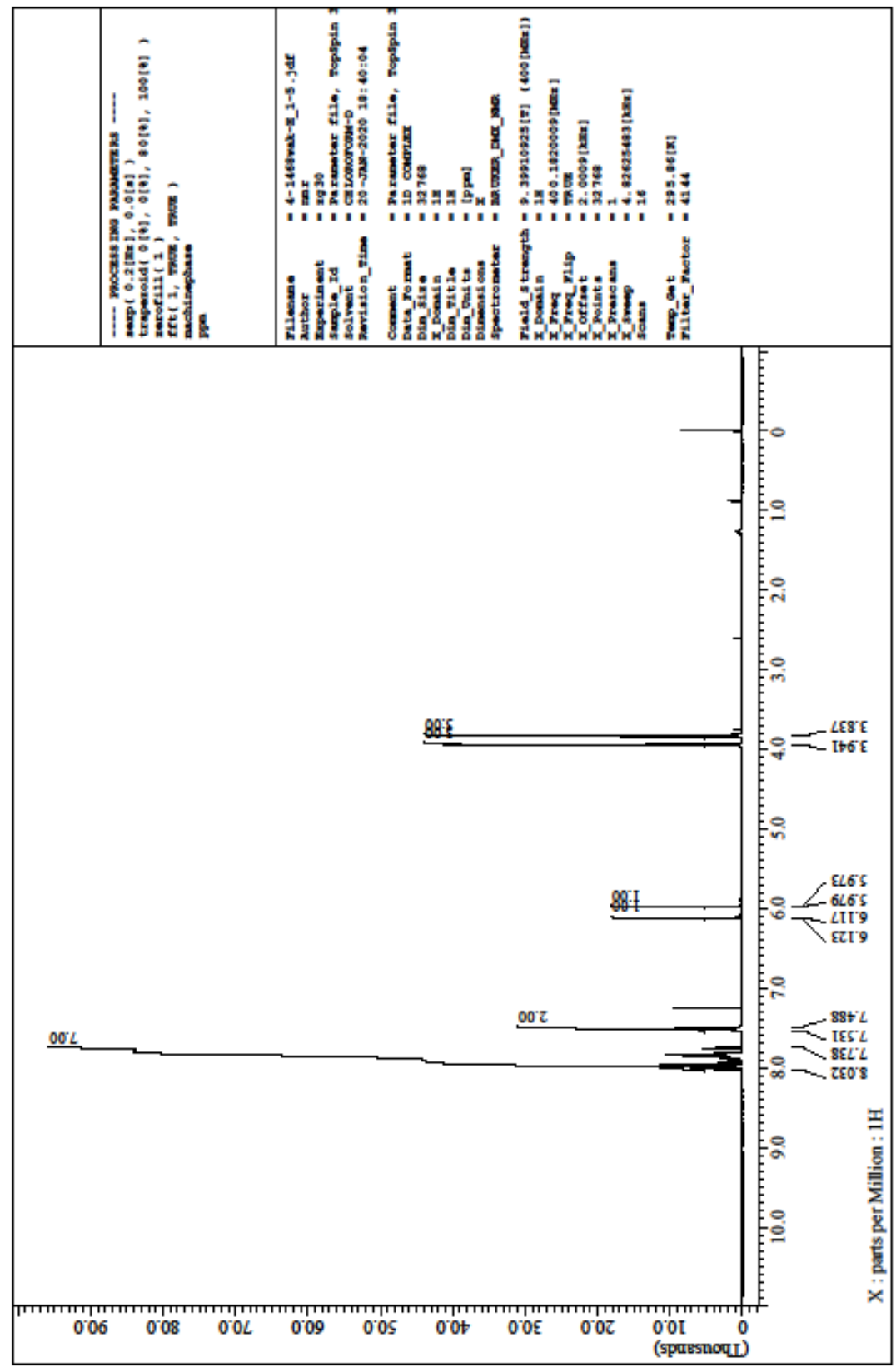


Figure $50 .{ }^{13} \mathrm{C}$ NMR of $3 \mathrm{~m}$

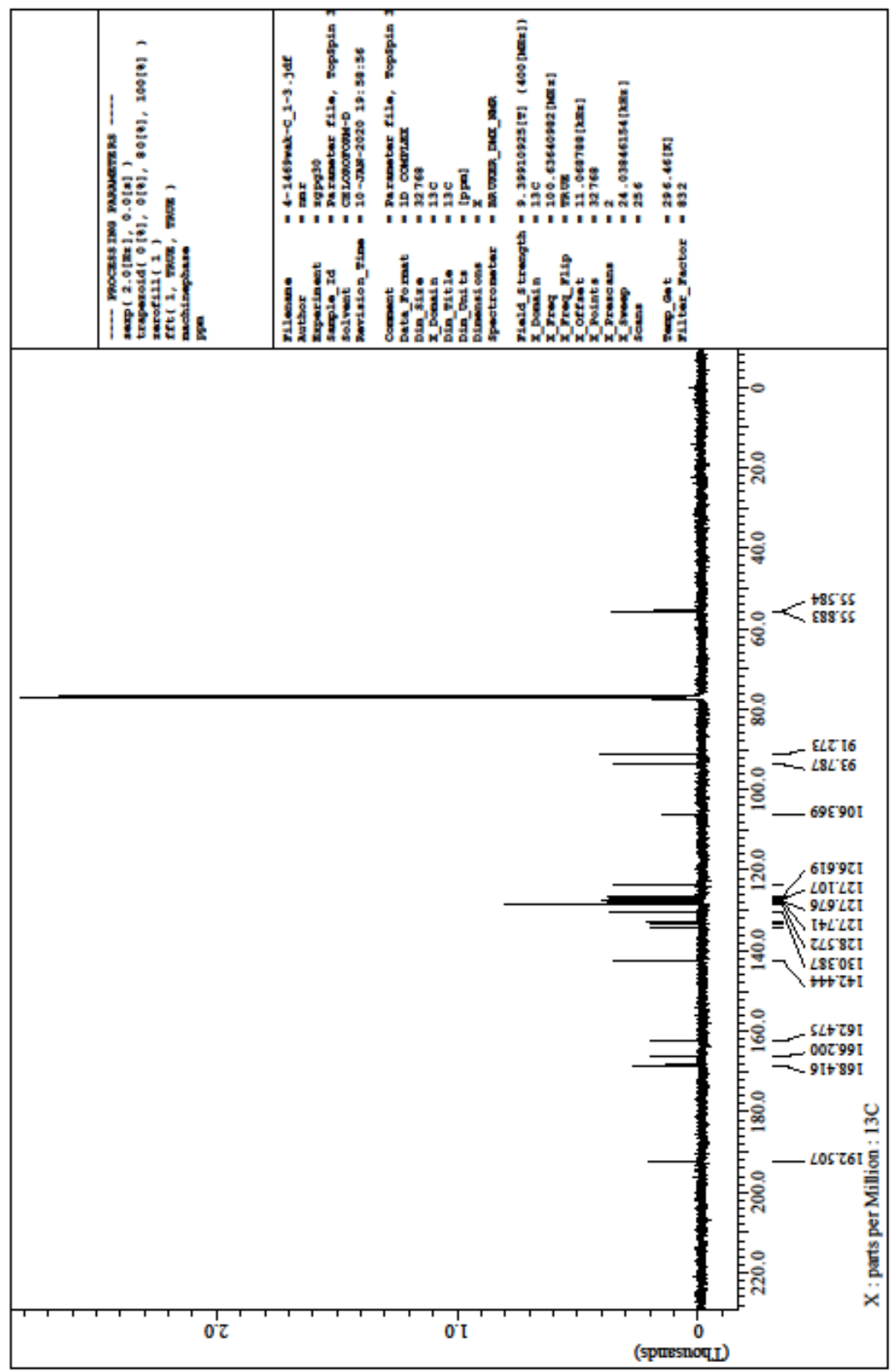


Figure 51. ${ }^{1} \mathrm{H}$ NMR of $4 \mathrm{a}$

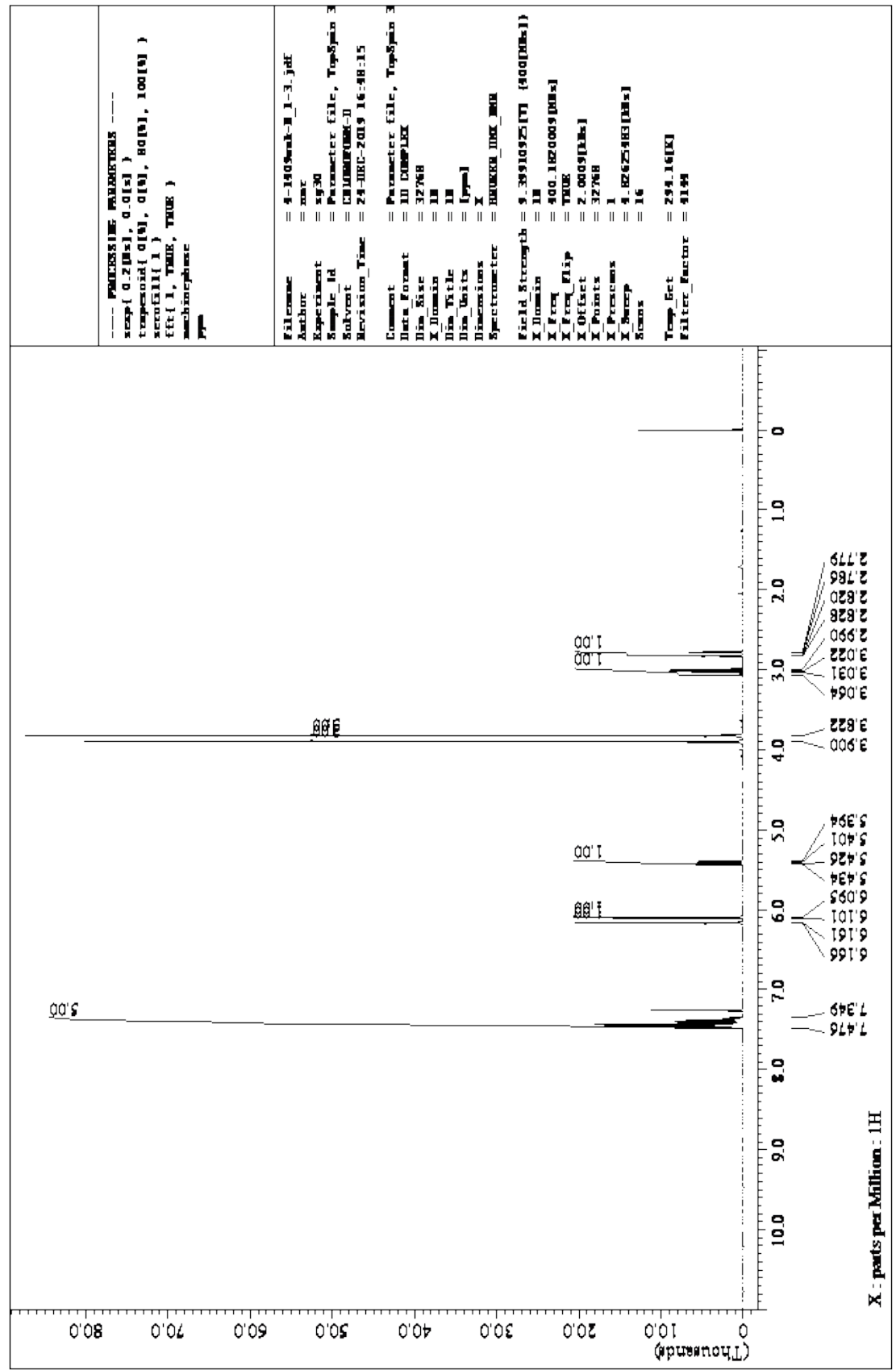


Figure 52. ${ }^{13} \mathrm{C}$ NMR of $4 \mathrm{a}$

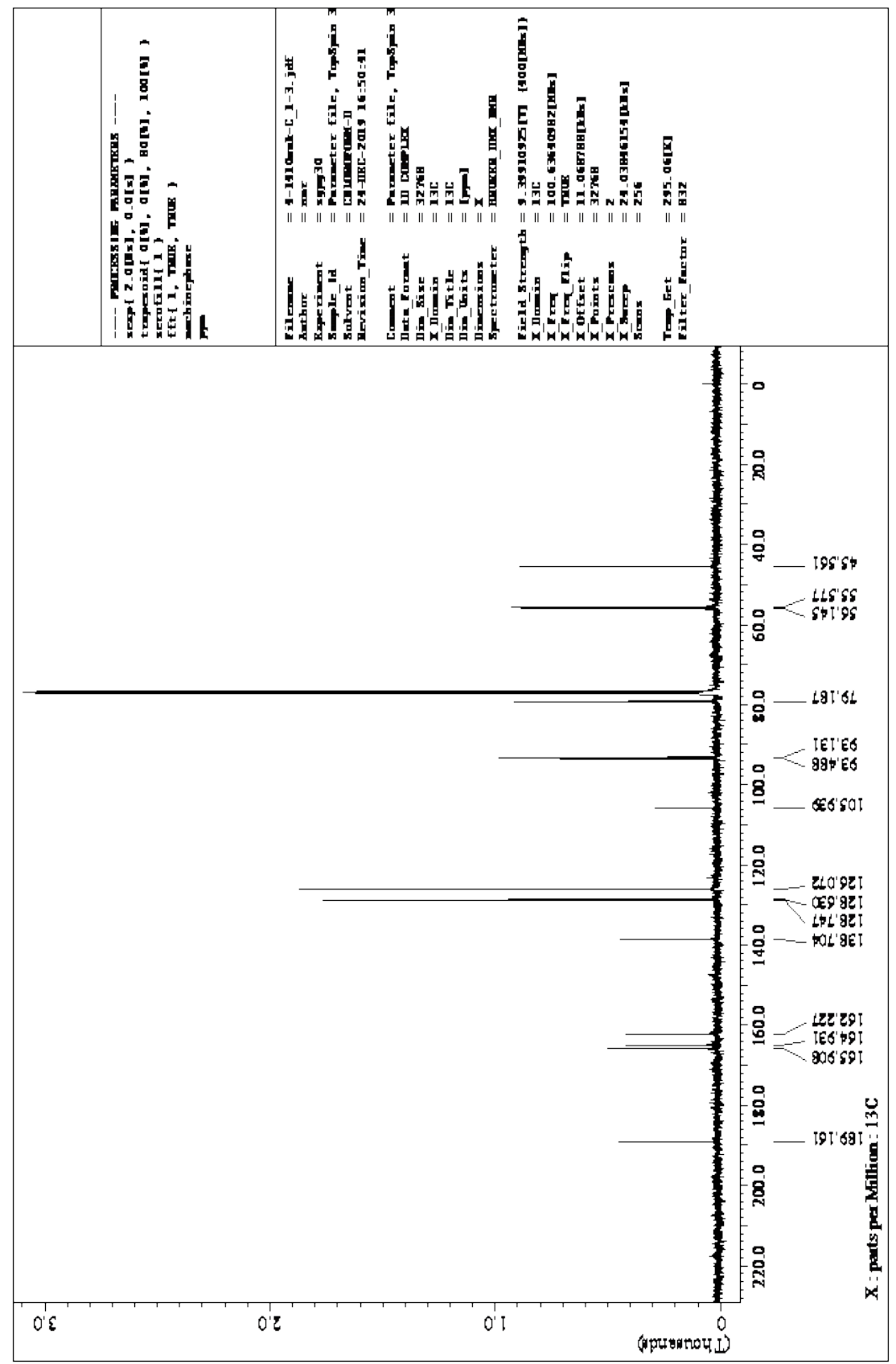


Figure 53. ${ }^{1} \mathrm{H}$ NMR of $4 b$

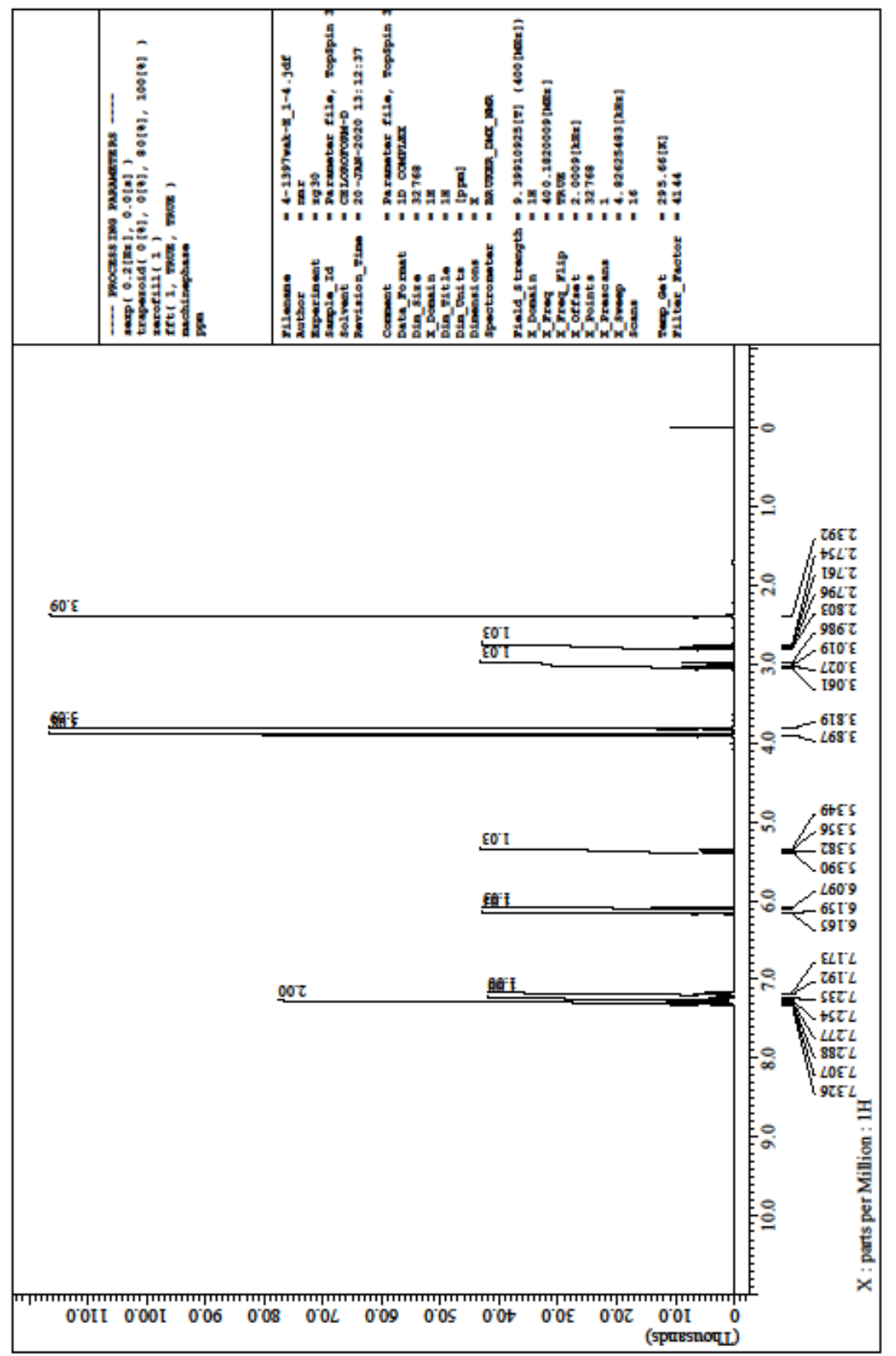


Figure $54 .{ }^{13} \mathrm{C}$ NMR of $4 \mathrm{~b}$

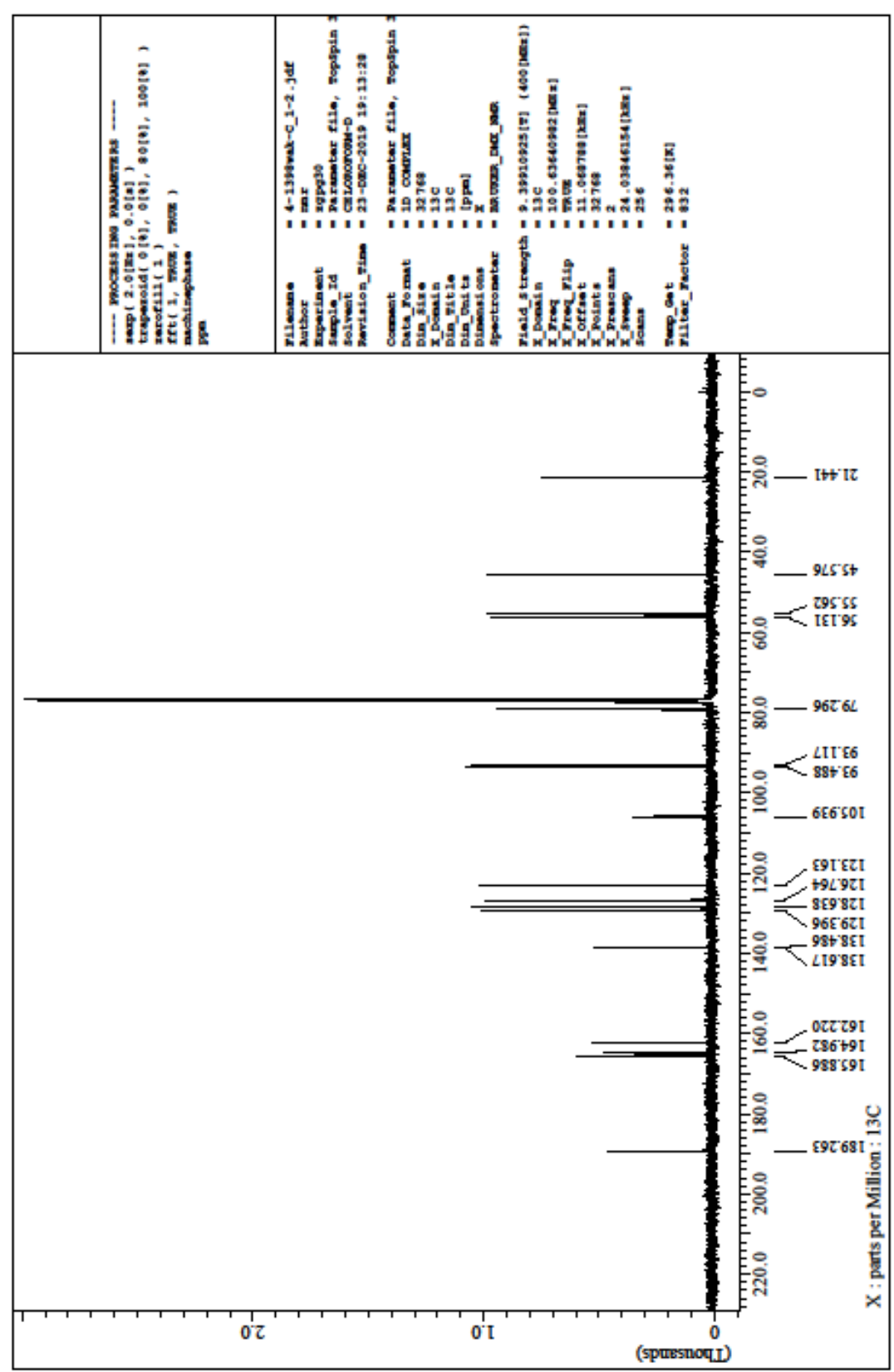


Figure 55. ${ }^{1} \mathrm{H}$ NMR of $4 \mathrm{c}$

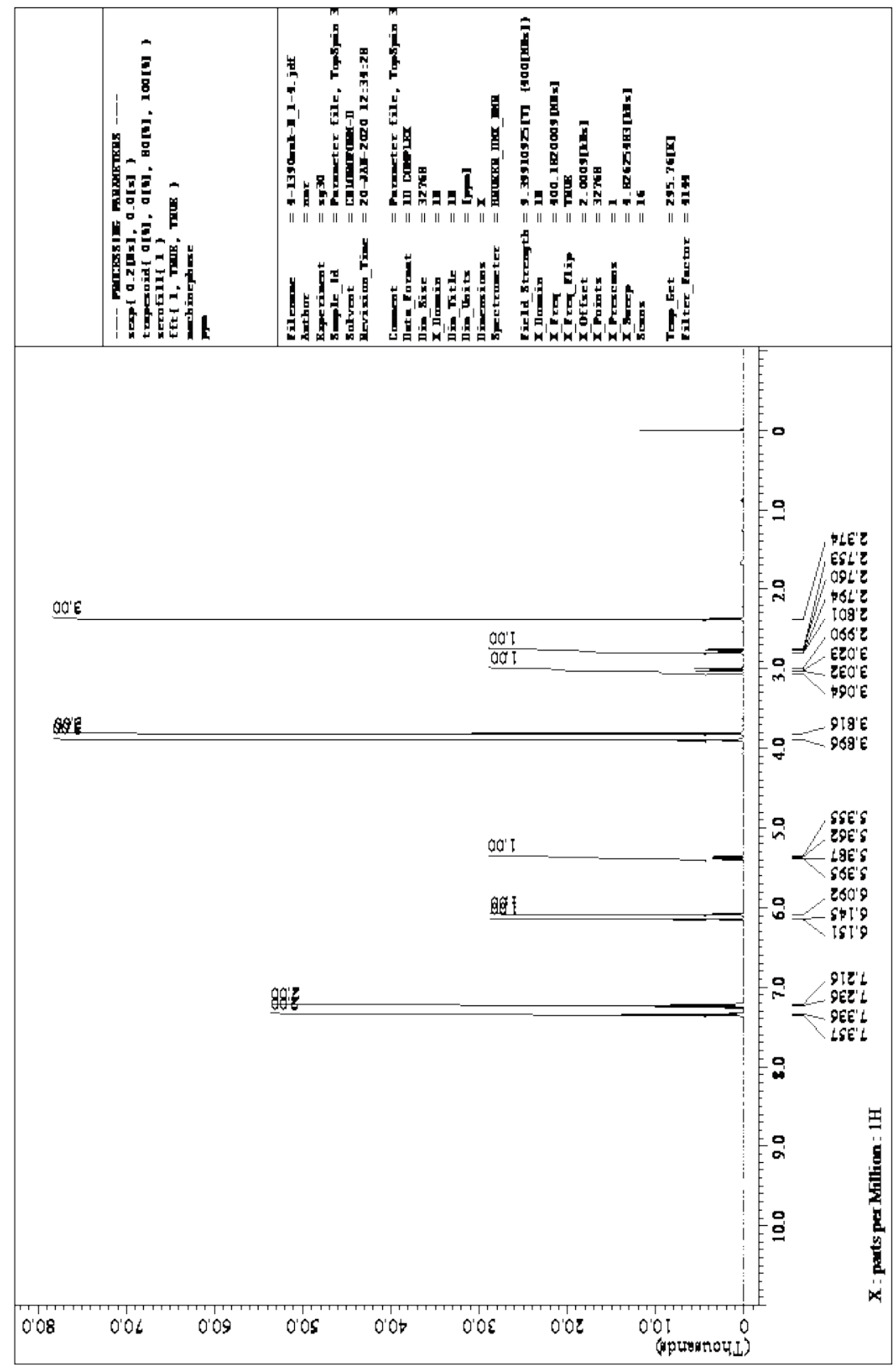


Figure 56. ${ }^{13} \mathrm{C}$ NMR of $4 \mathrm{c}$

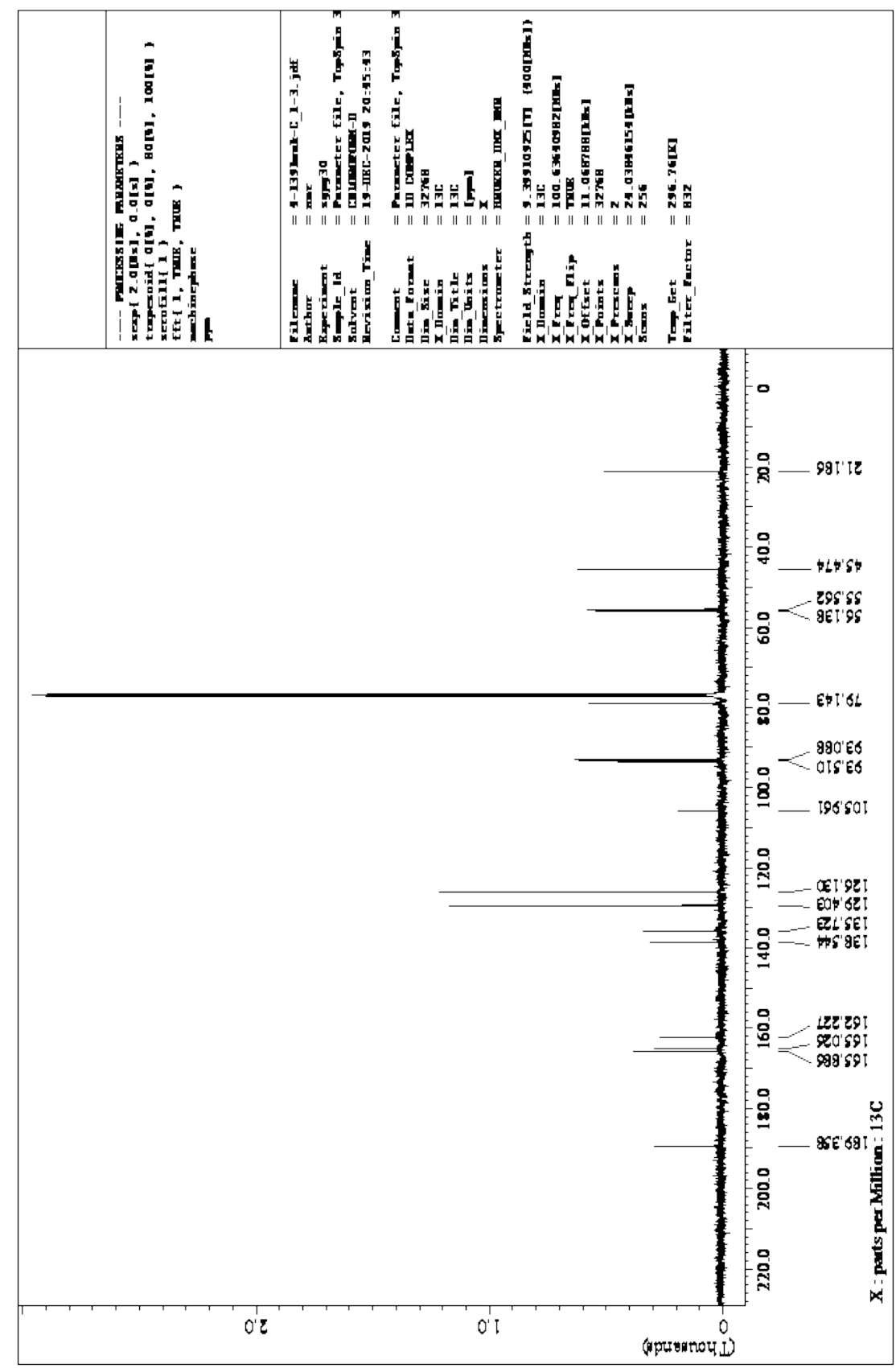


Figure 57. ${ }^{1} \mathrm{H}$ NMR of $4 \mathrm{~d}$

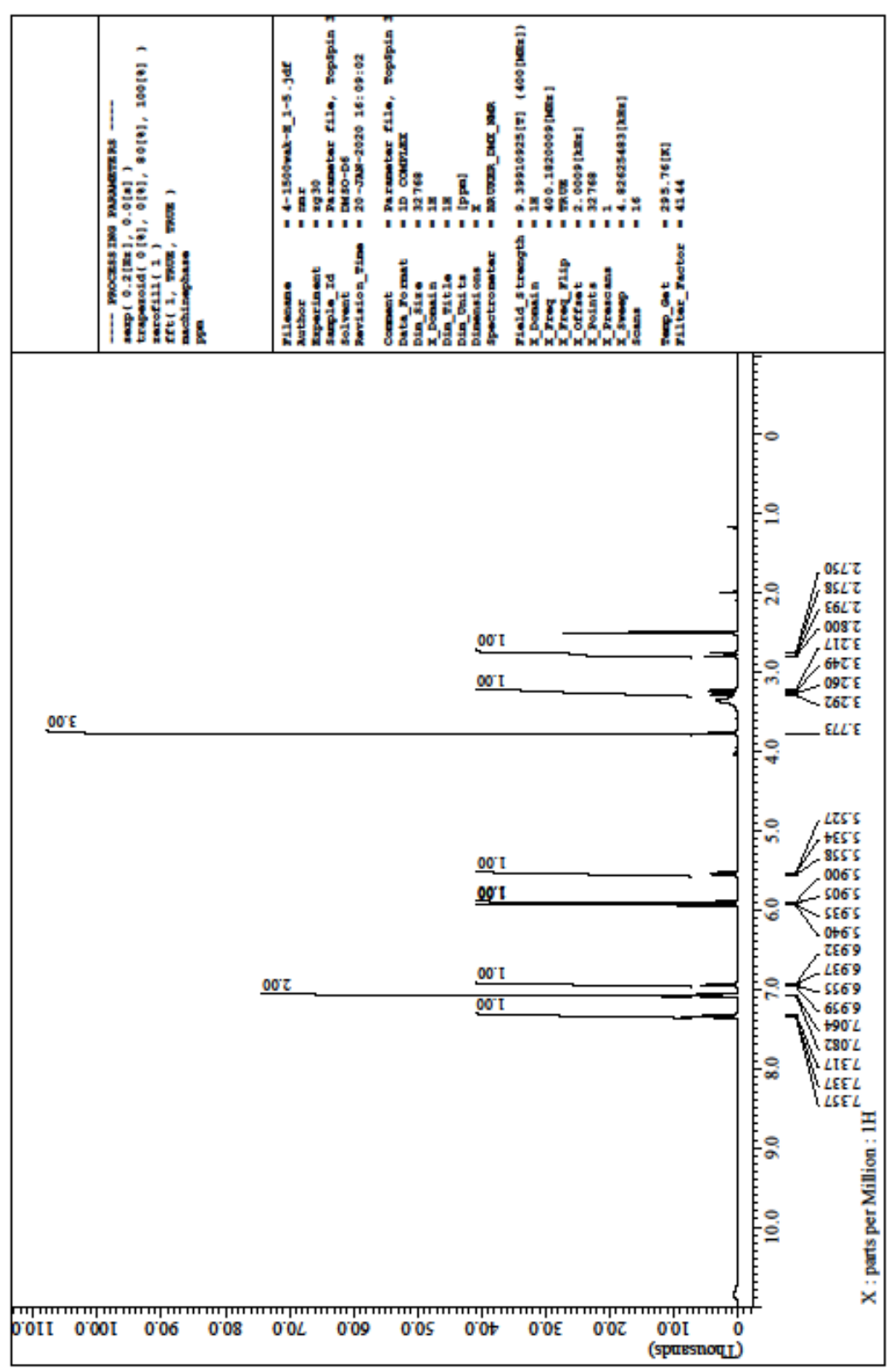


Figure 58. ${ }^{13} \mathrm{C}$ NMR of $4 d$

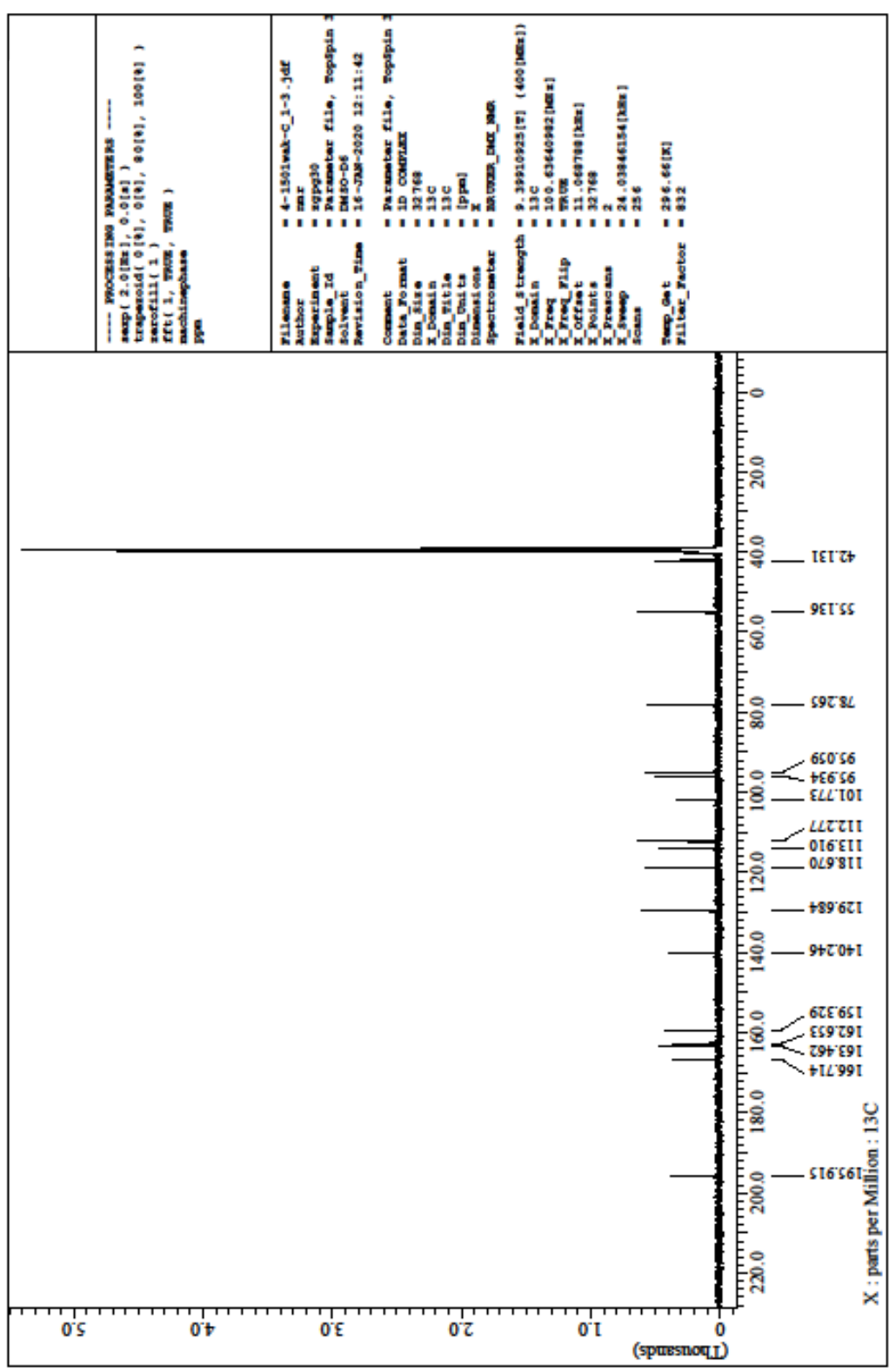


Figure 59. ${ }^{1} \mathrm{H}$ NMR of $4 \mathrm{e}$

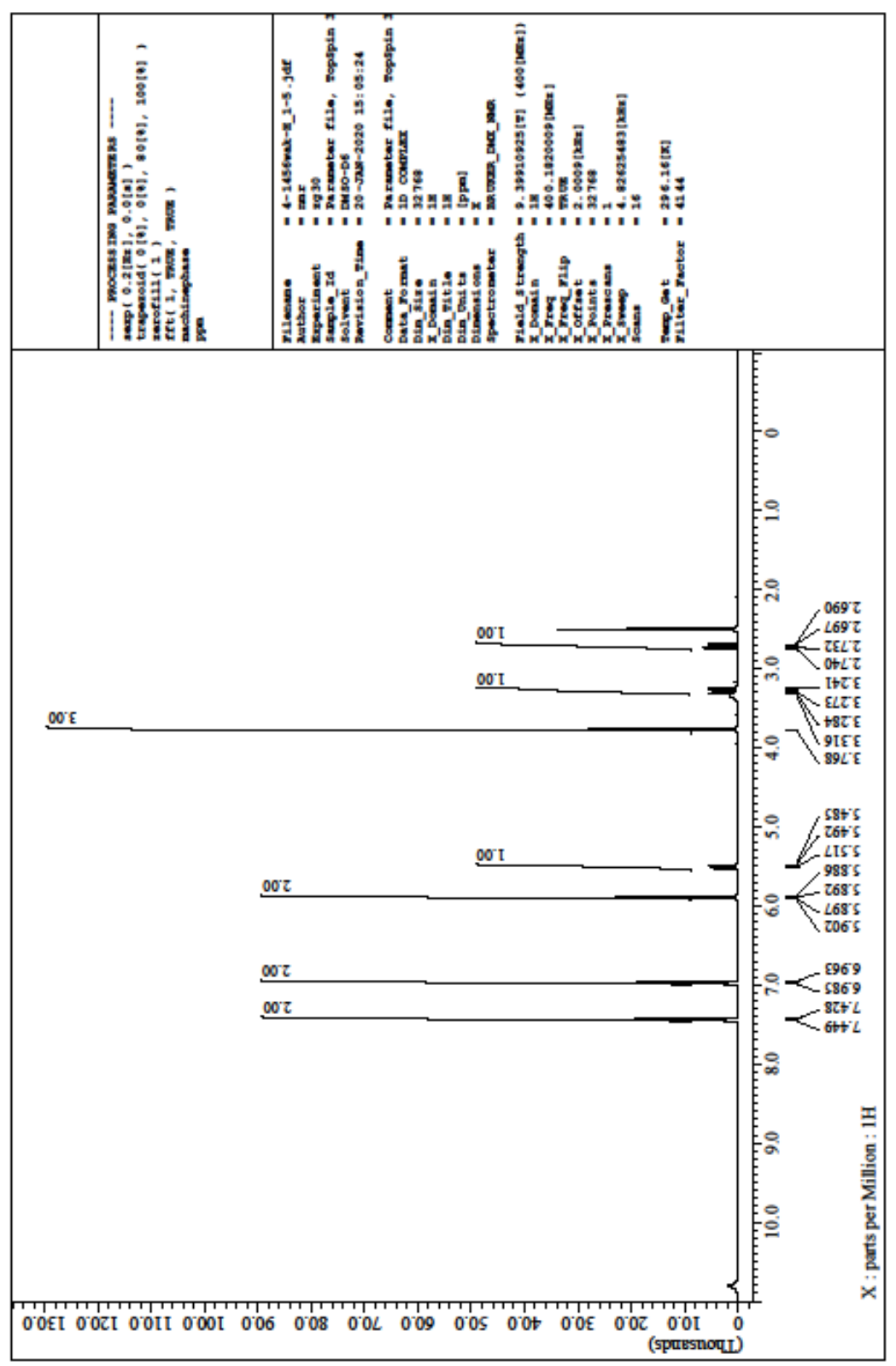


Figure $60 .{ }^{13} \mathrm{C}$ NMR of $4 e$

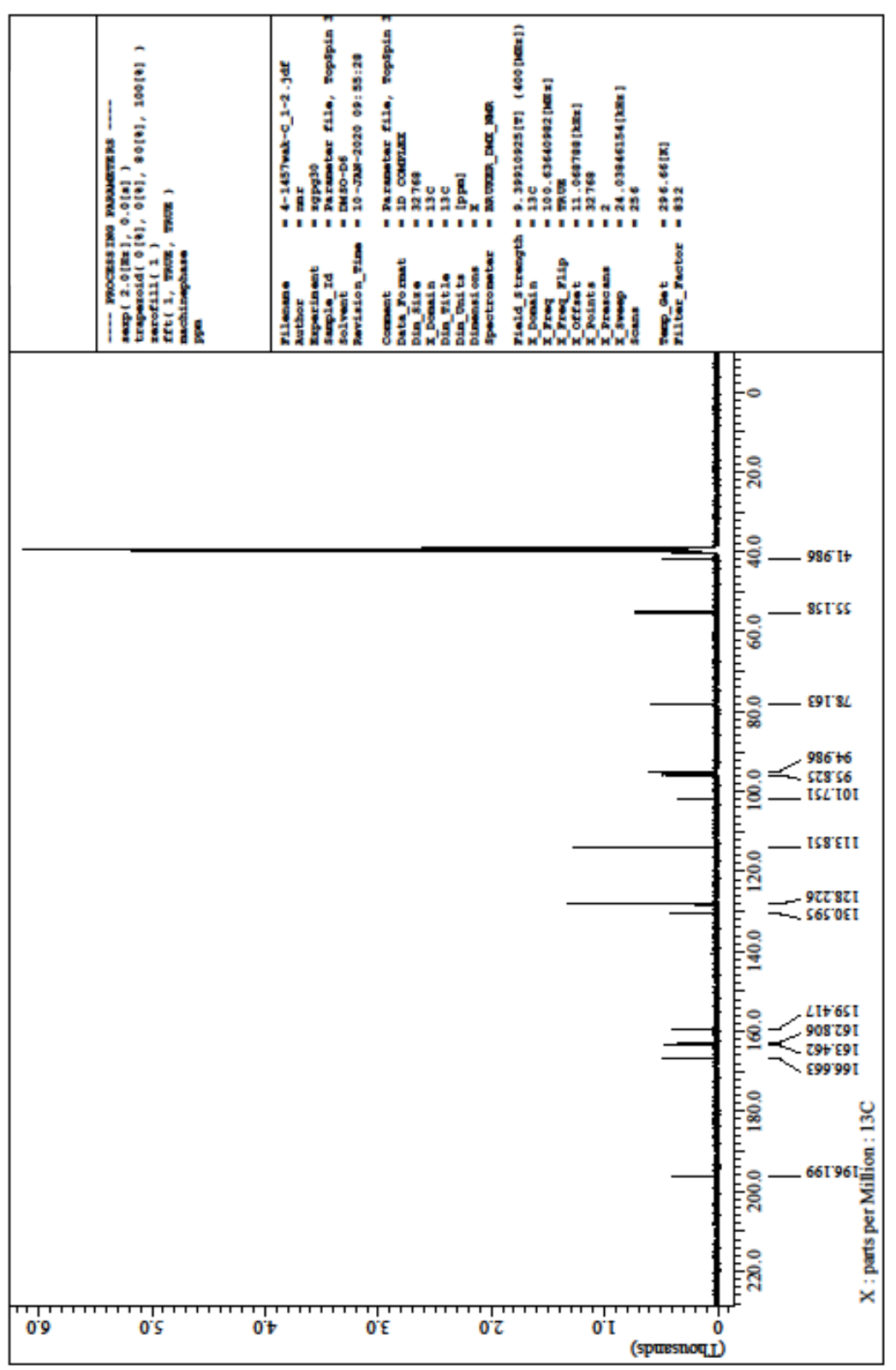


Figure 61. ${ }^{1} \mathrm{H}$ NMR of $4 \mathrm{f}$

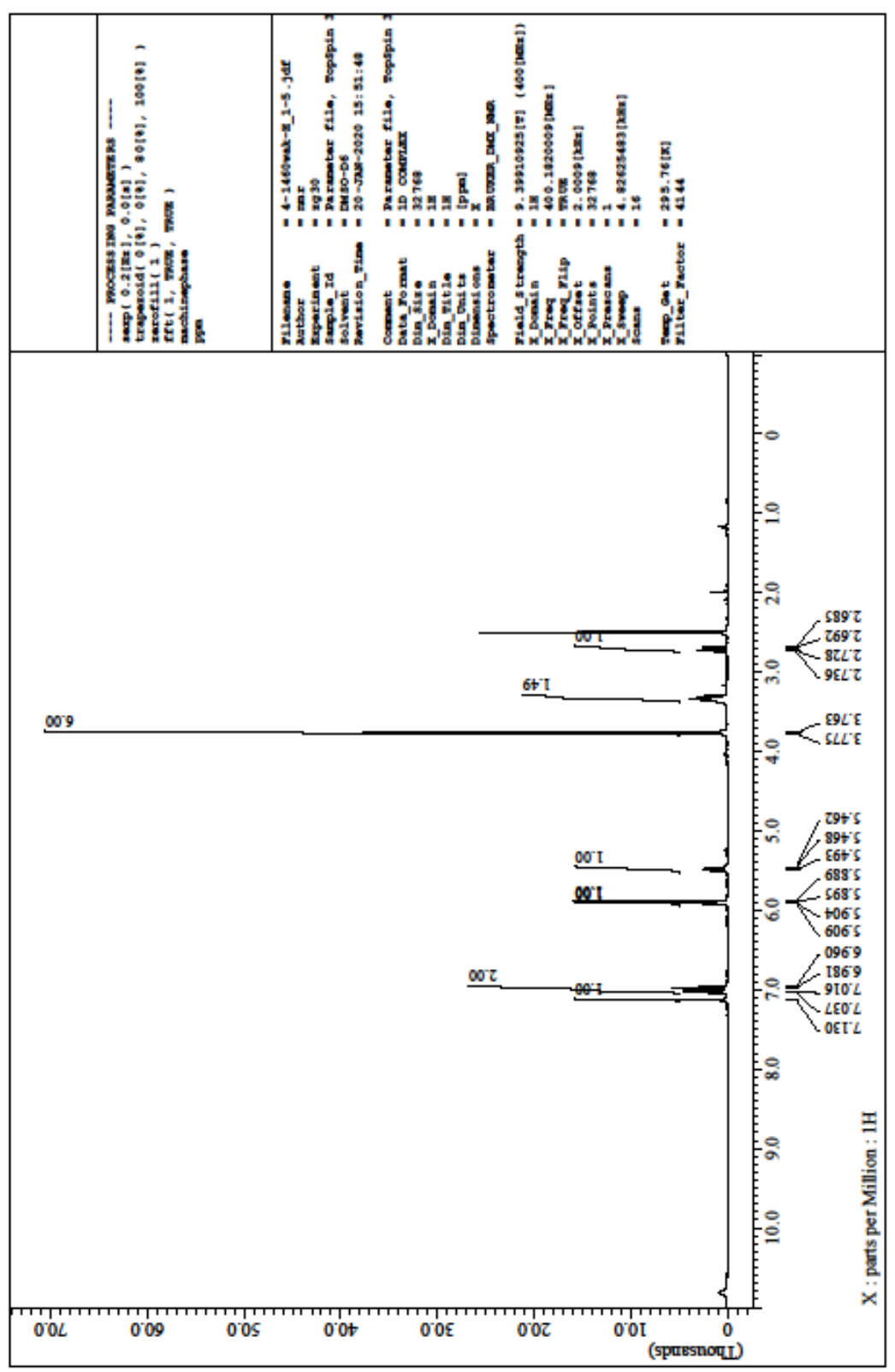


Figure $62 .{ }^{13} \mathrm{C}$ NMR of $\mathbf{4 f}$

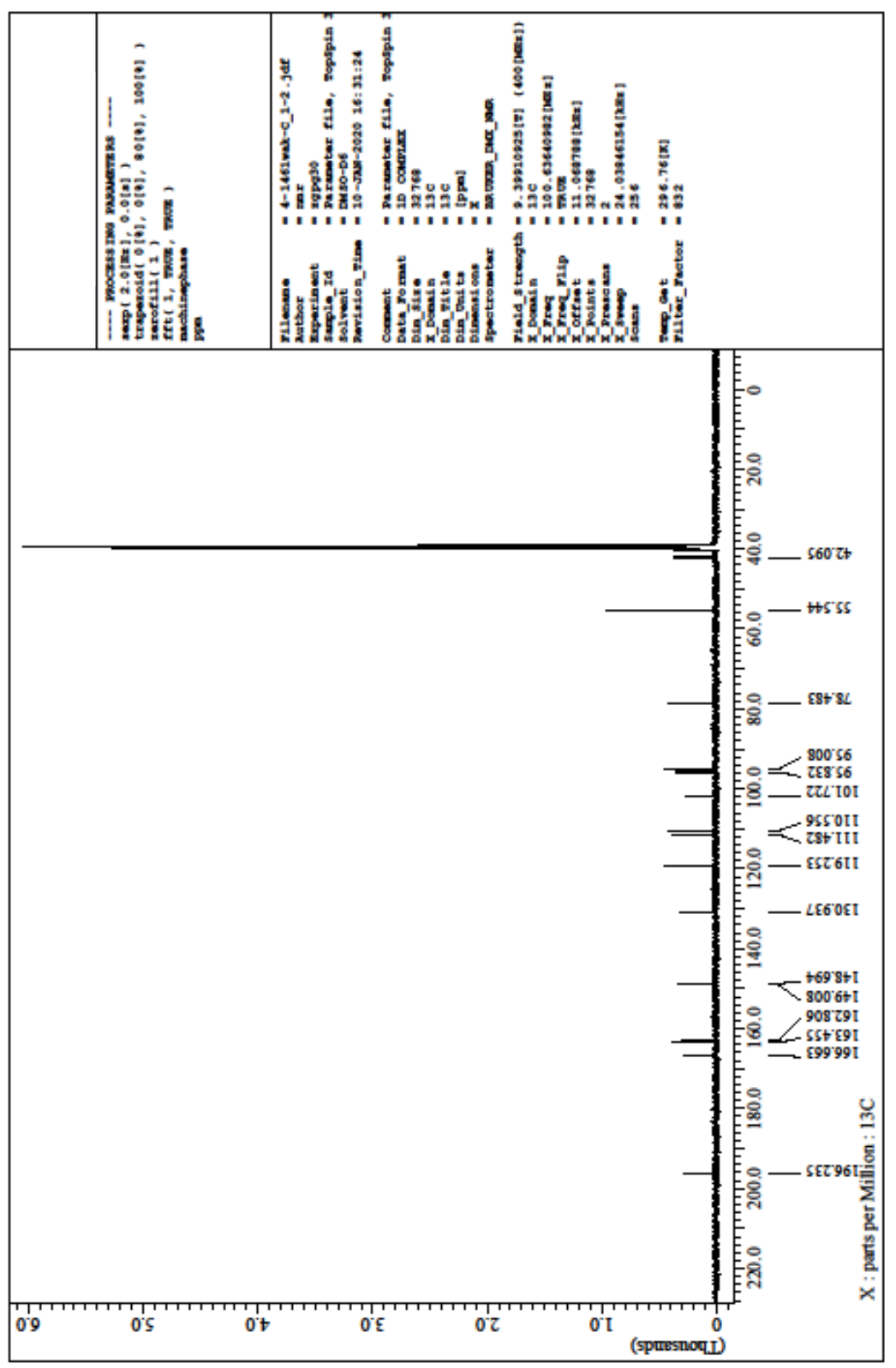


Figure 63. ${ }^{1} \mathrm{H}$ NMR of $\mathbf{4 g}$

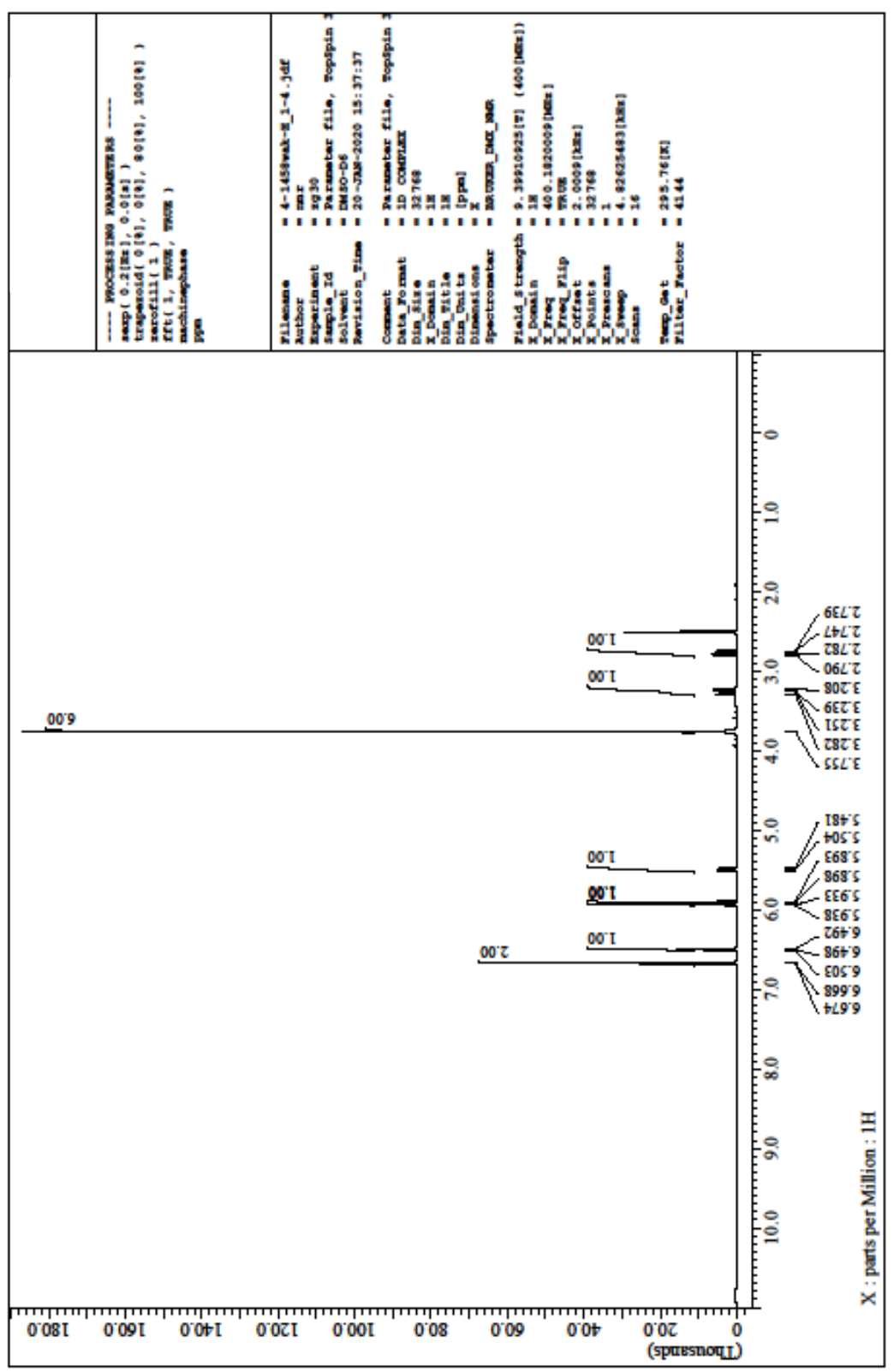


Figure $64 .{ }^{13} \mathrm{C}$ NMR of $4 \mathrm{~g}$

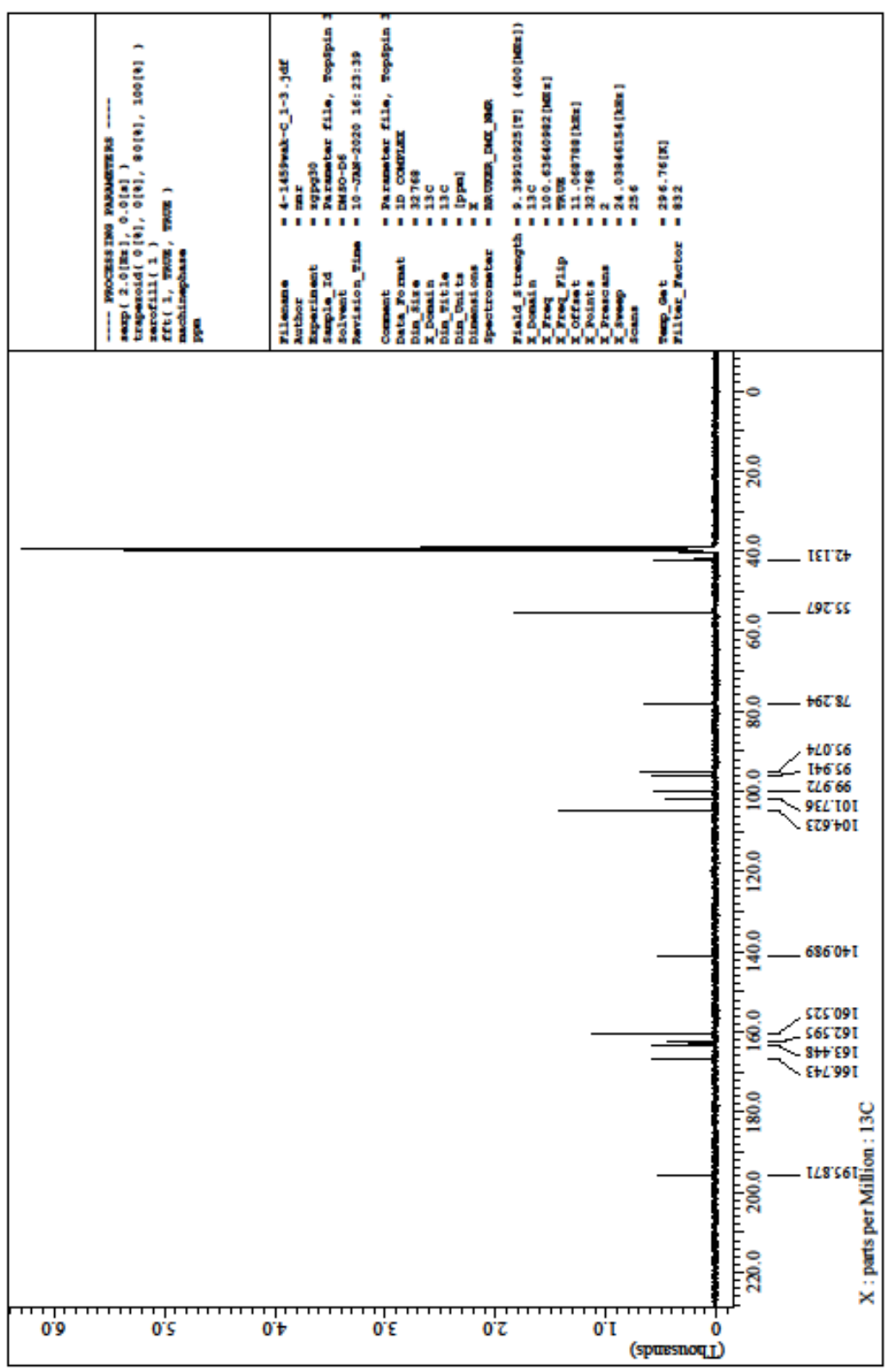


Figure 65. ${ }^{1} \mathrm{H}$ NMR of $4 \mathrm{~h}$

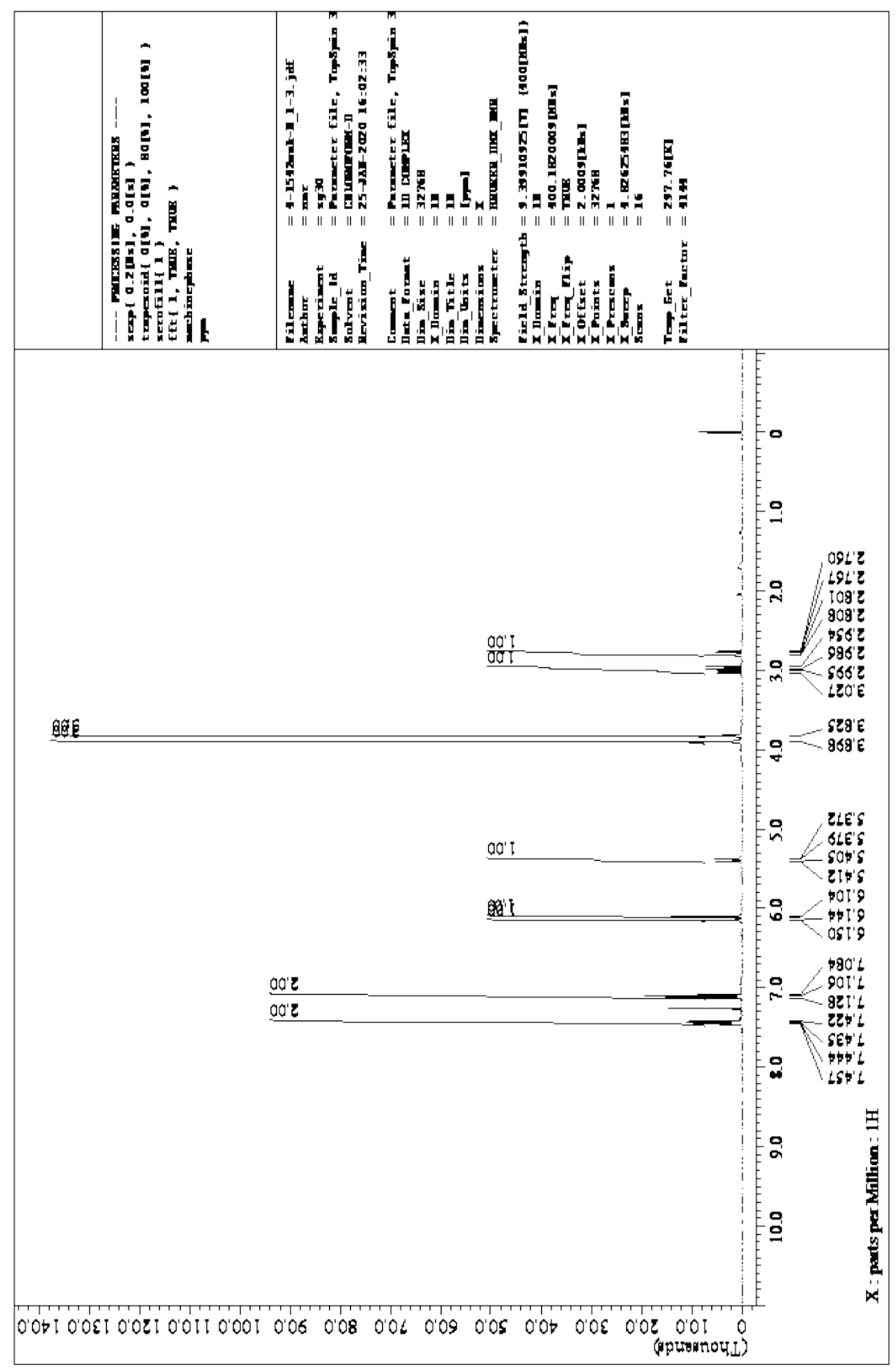


Figure $66 .{ }^{13} \mathrm{C}$ NMR of $4 \mathrm{~h}$

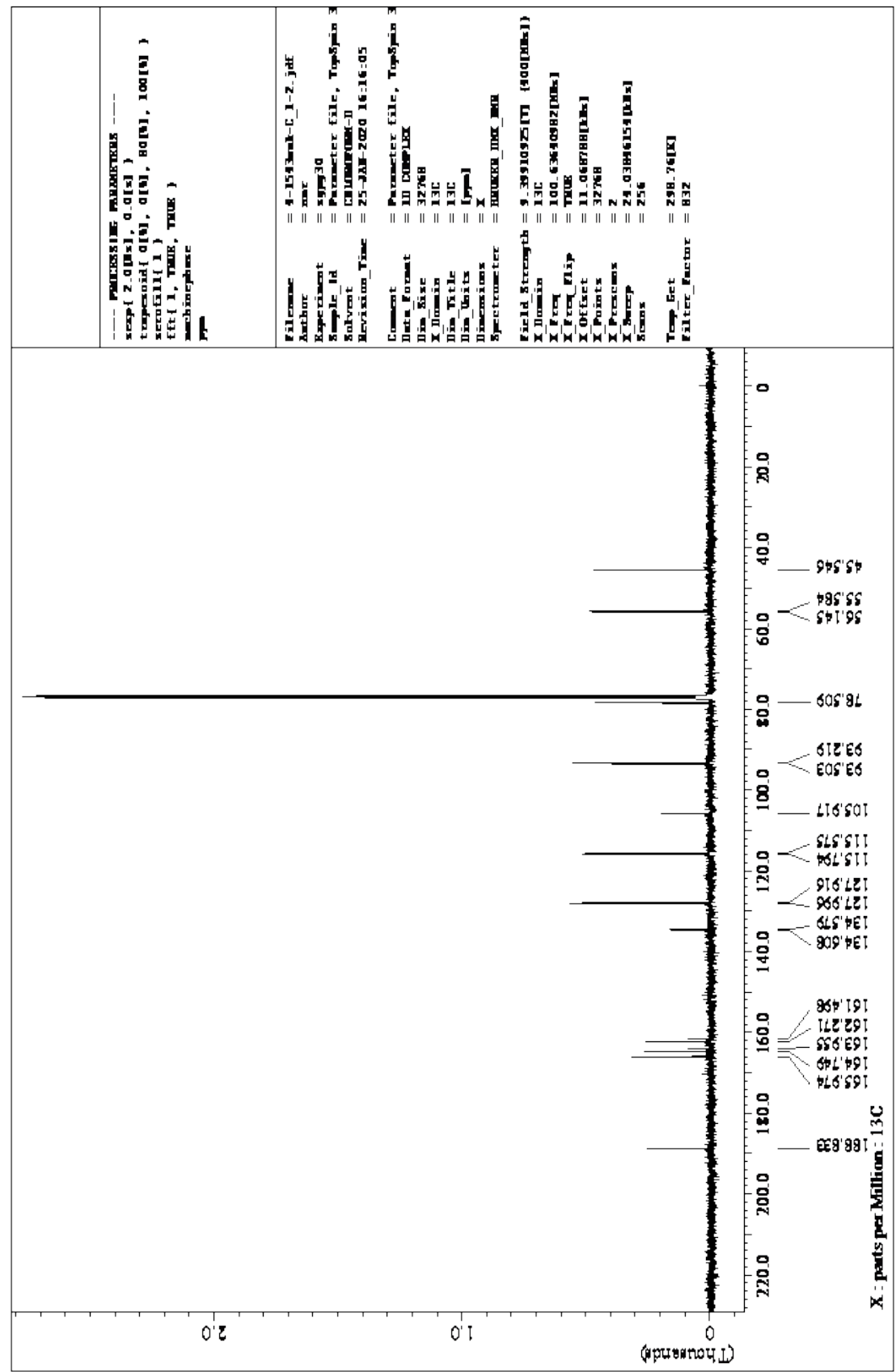


Figure $67 .{ }^{1} \mathrm{H}$ NMR of $4 \mathrm{i}$

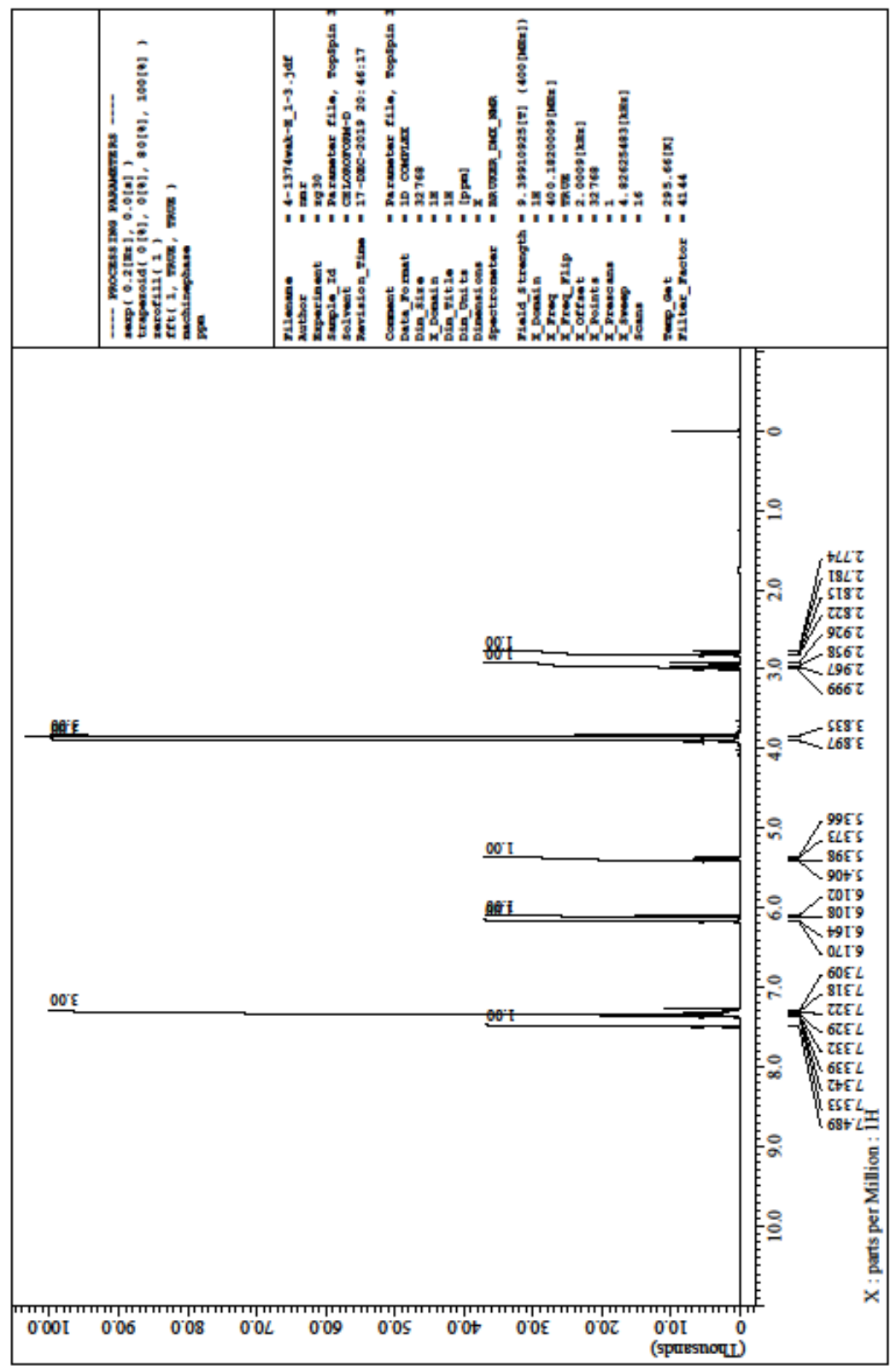


Figure $68 .{ }^{13} \mathrm{C}$ NMR of $4 \mathbf{i}$

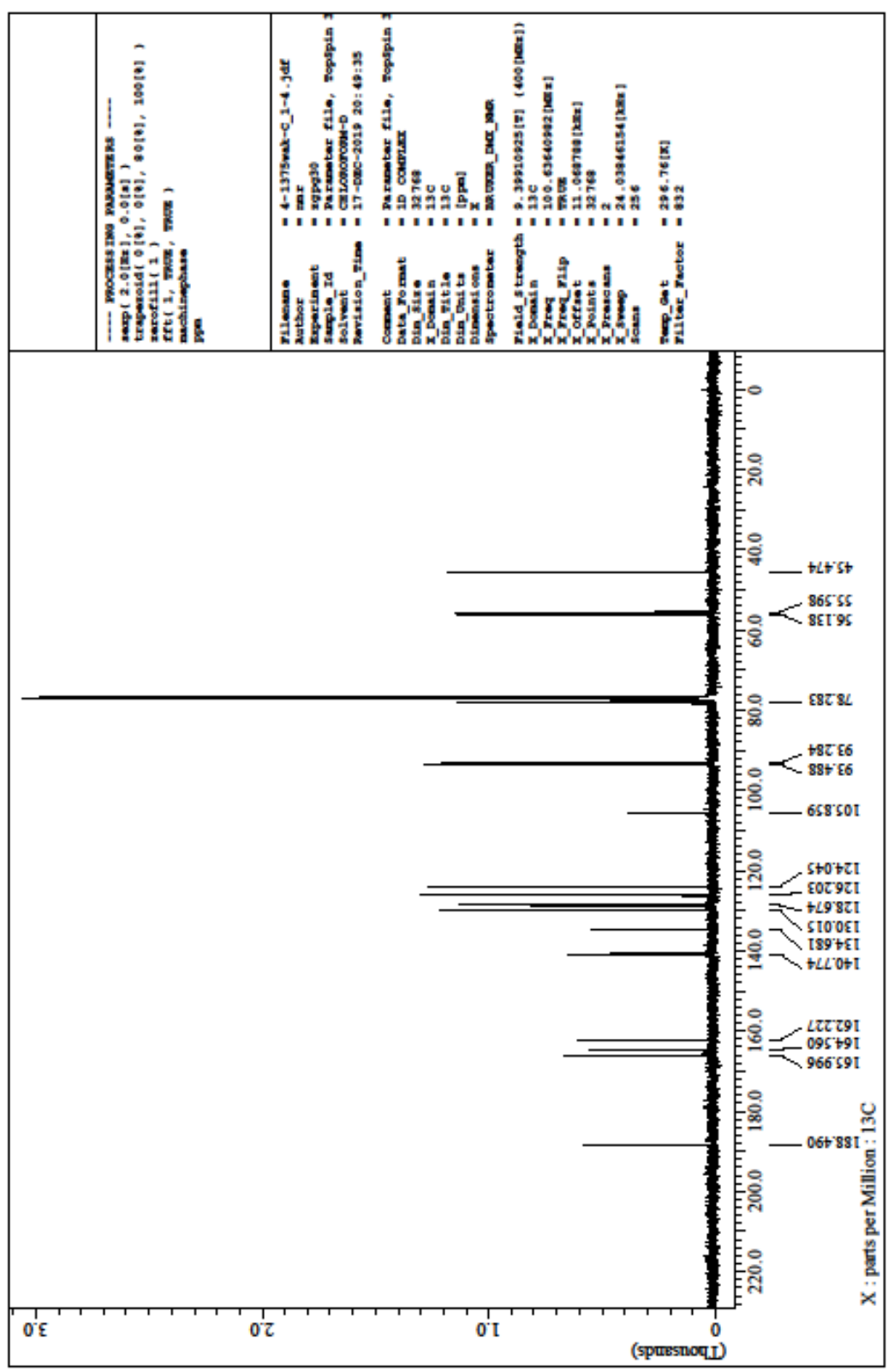


Figure 69. ${ }^{1} \mathrm{H}$ NMR of $4 \mathbf{j}$

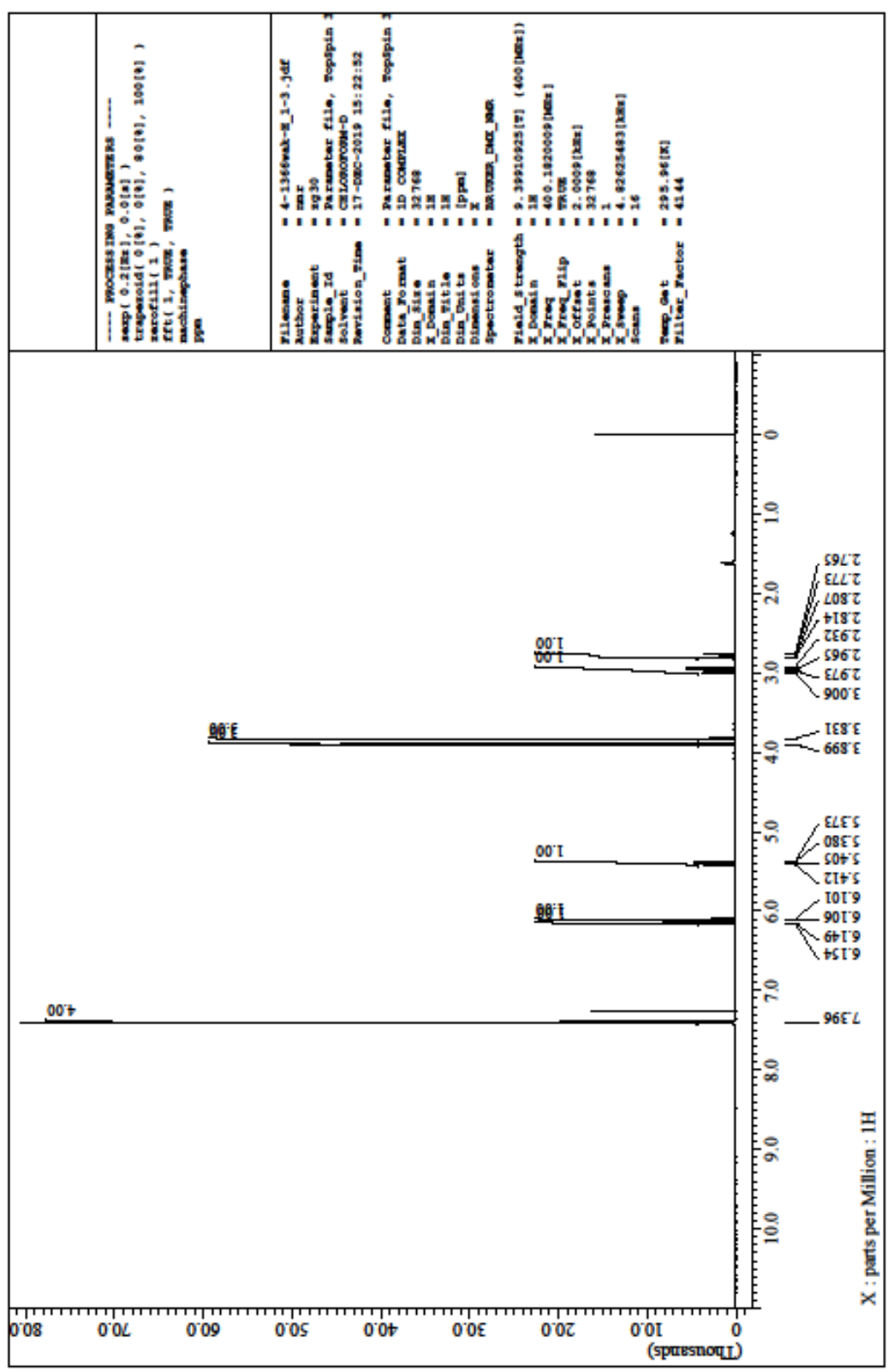


Figure $70 .{ }^{13} \mathrm{C}$ NMR of $\mathbf{4 j}$

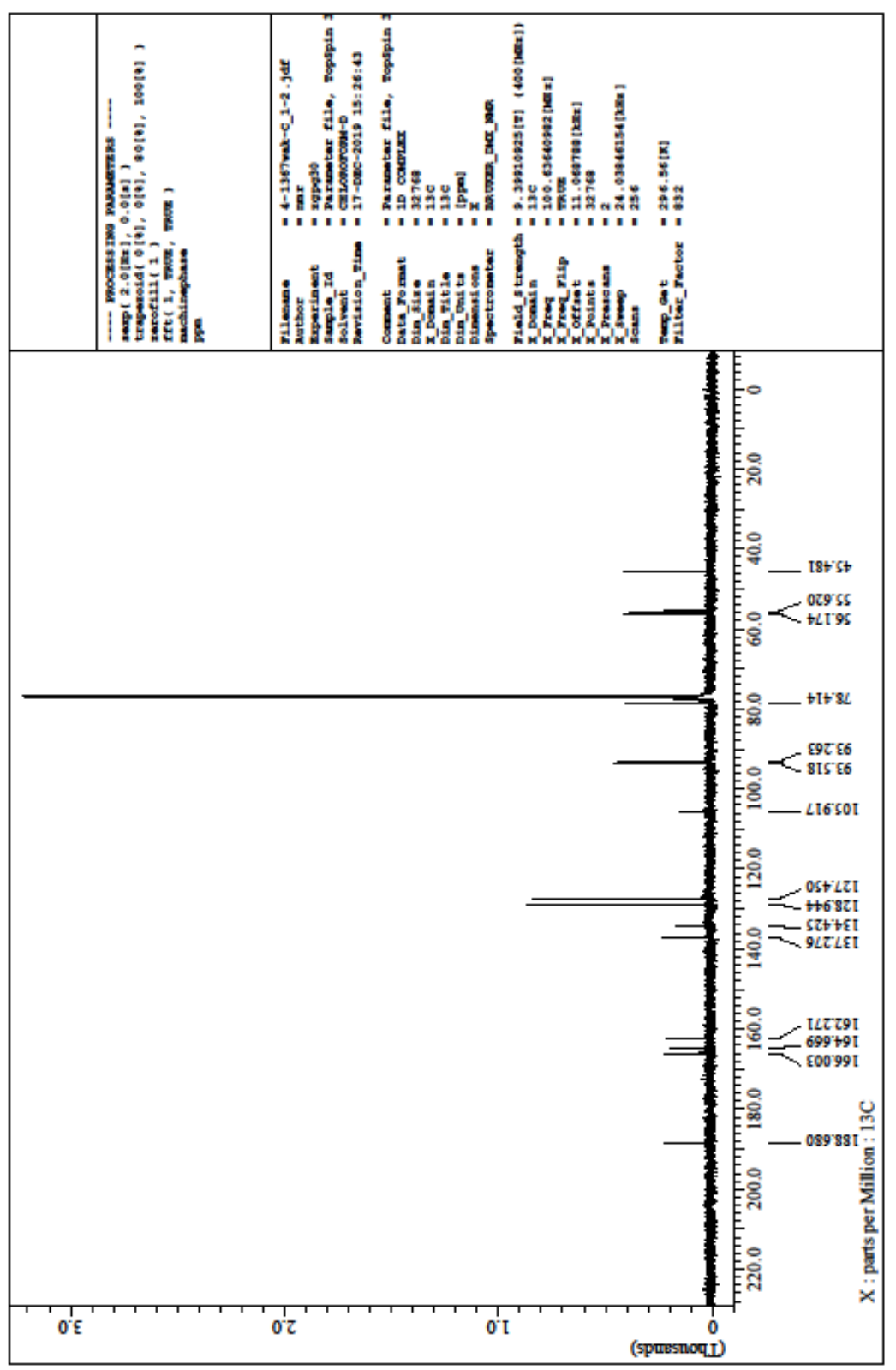


Figure 71. ${ }^{1} \mathrm{H}$ NMR of $4 k$

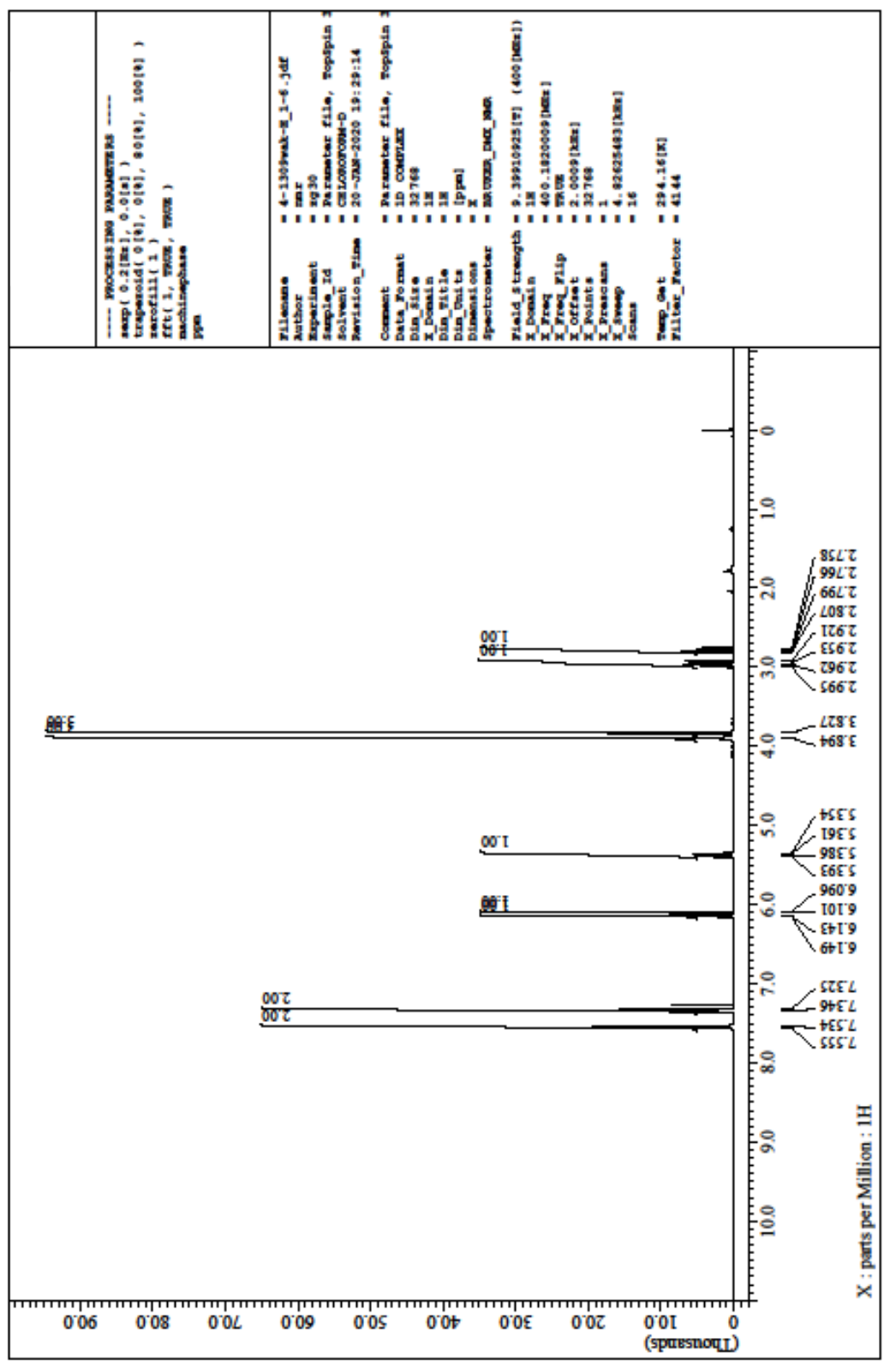


Figure $72 .{ }^{13} \mathrm{C}$ NMR of $4 \mathrm{k}$

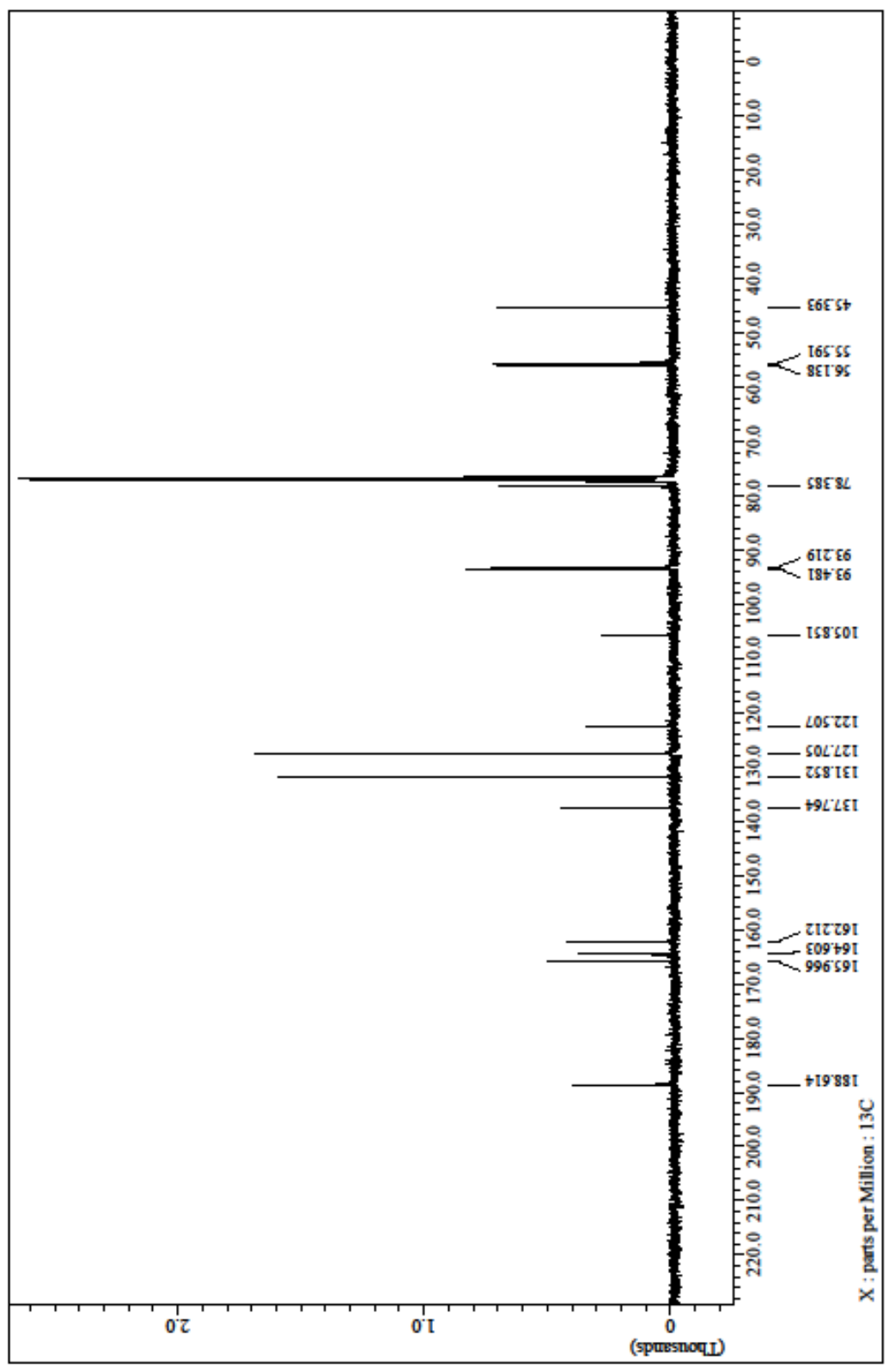


Figure 73. ${ }^{1} \mathrm{H}$ NMR of $4 \mathrm{I}$

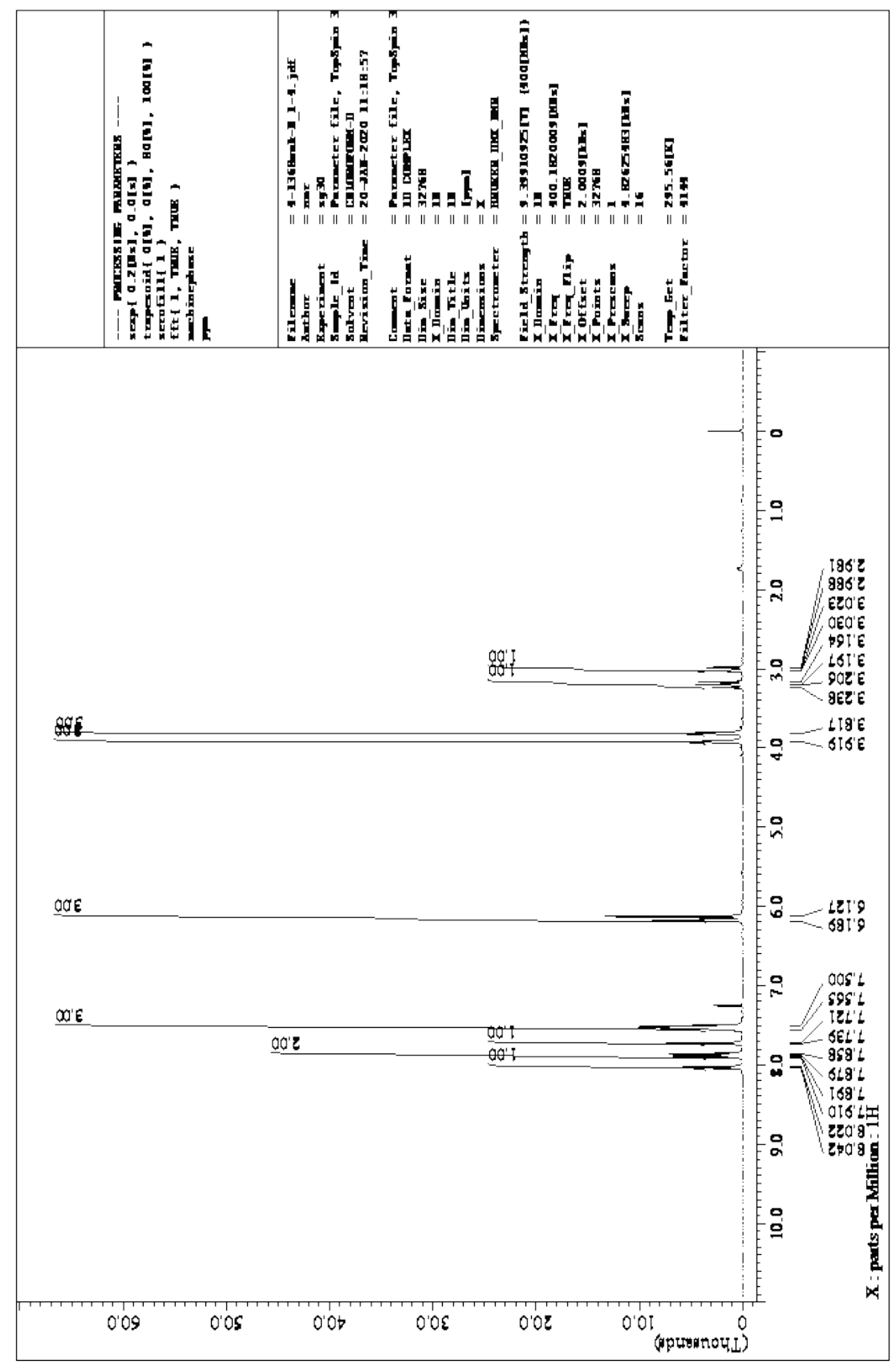


Figure $74 .{ }^{13} \mathrm{C}$ NMR of 4 I

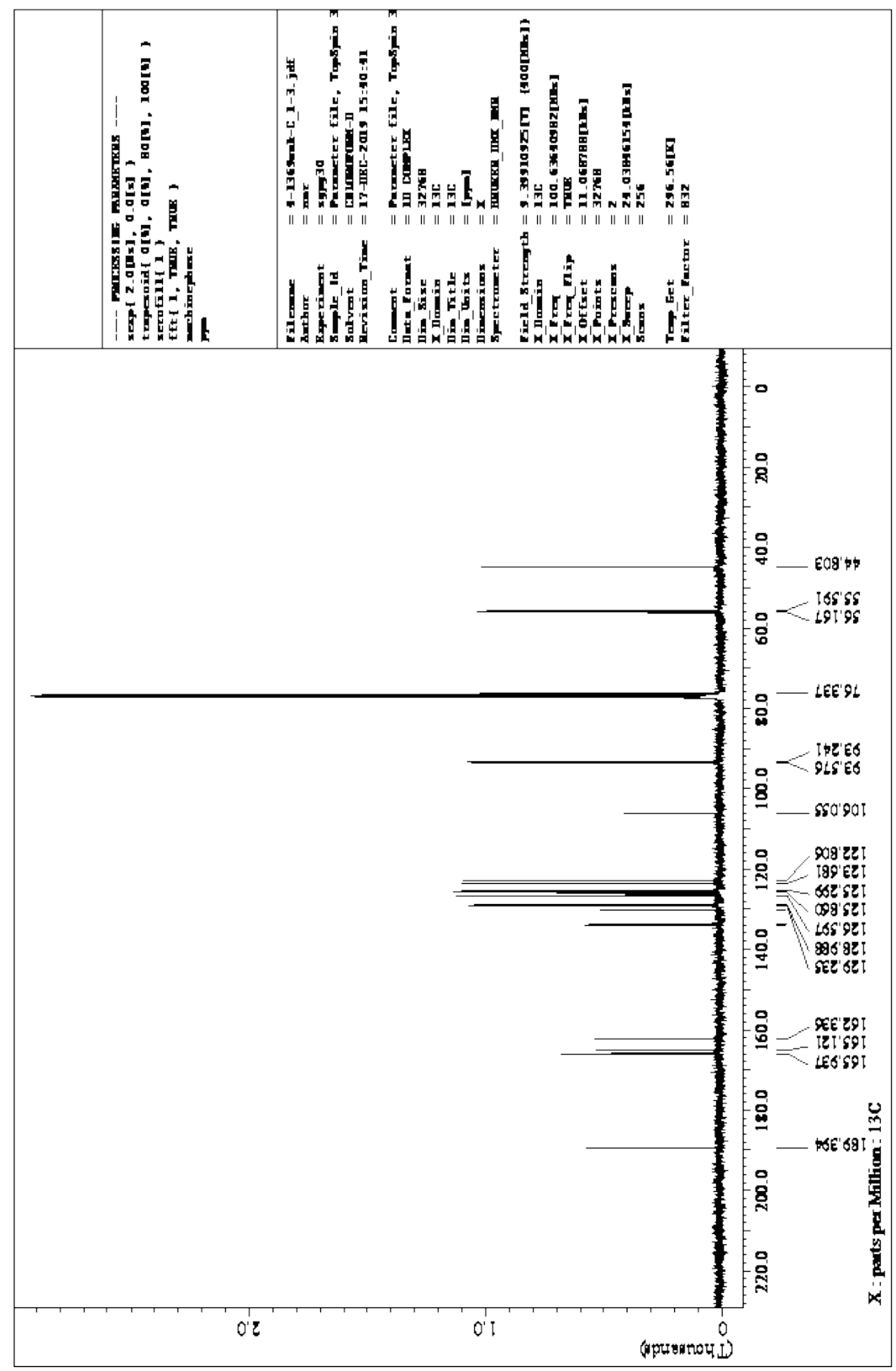


Figure 75. ${ }^{1} \mathrm{H}$ NMR of $4 \mathrm{~m}$

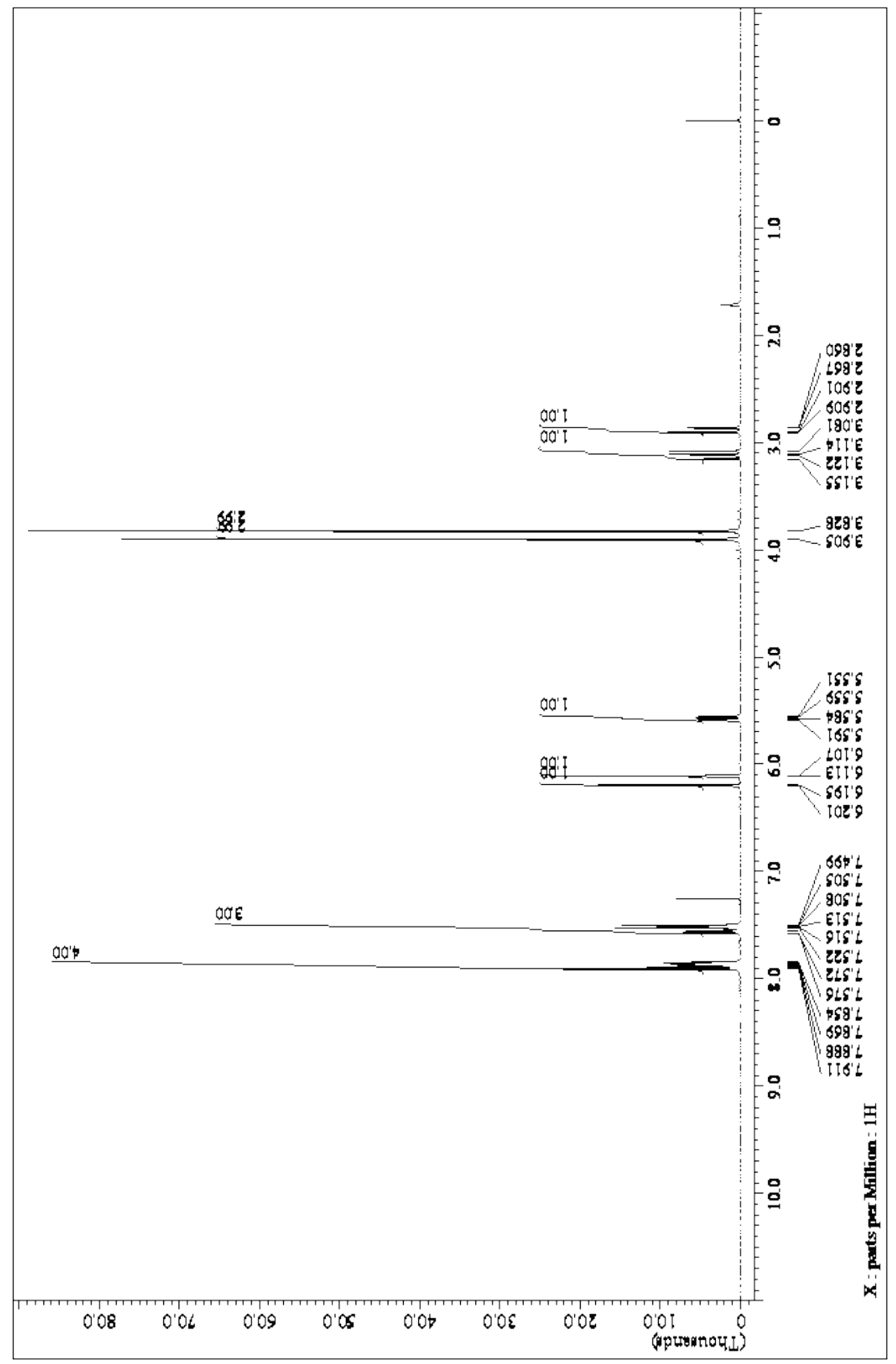


Figure 76. ${ }^{13} \mathrm{C}$ NMR of $4 m$

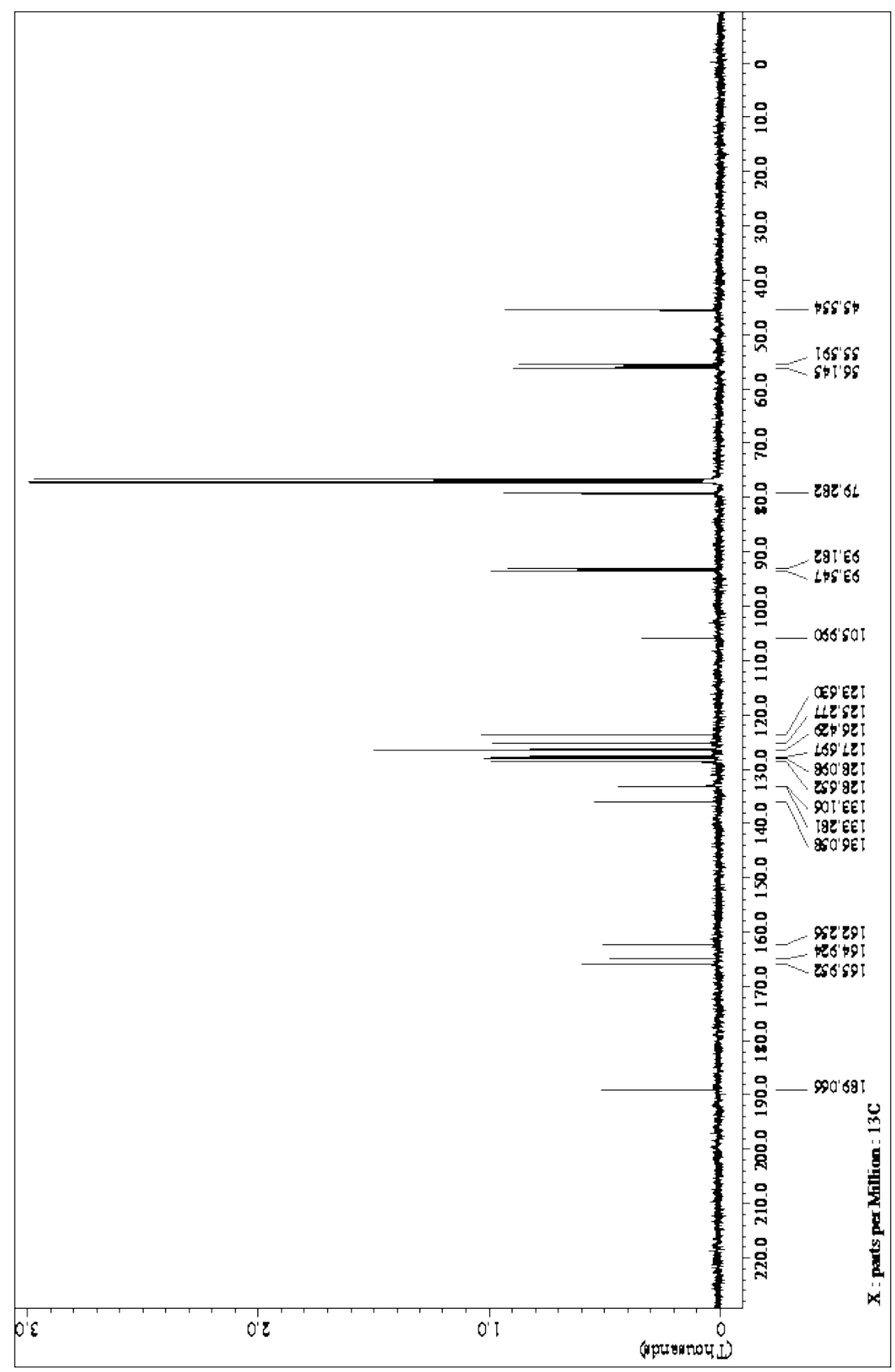

